DRAFT VERSION NOVEMBER 23, 2018

Preprint typeset using LATEX style emulateapj v. 11/10/09

\title{
SPECTRAL SURVEY OF X-RAY BRIGHT ACTIVE GALACTIC NUCLEI FROM THE ROSSI X-RAY TIMING EXPLORER
}

\author{
Elizabeth Rivers ${ }^{1}$, Alex MARKowitz ${ }^{1}$, AND Richard RothsCHILD ${ }^{1}$ \\ Draft version November 23, 2018
}

\begin{abstract}
Using long-term monitoring data from the Rossi X-ray Timing Explorer (RXTE), we have selected 23 active galactic nuclei (AGN) with sufficient brightness and overall observation time to derive broadband X-ray spectra from 3 to $\gtrsim 100 \mathrm{keV}$. Our sample includes mainly radio-quiet Seyferts, as well as seven radio-loud sources. Given the longevity of the RXTE mission, the greater part of our data is spread out over more than a decade, providing truly long-term average spectra and eliminating inconsistencies arising from variability. We present long-term average values of absorption, Fe line parameters, Compton reflection strengths and photon indices, as well as fluxes and luminosities for the hard and very hard energy bands, $2-10 \mathrm{keV}$ and $20-100 \mathrm{keV}$ respectively. We find tentative evidence for high-energy rollovers in three of our objects. We improve upon previous surveys of the very hard X-ray energy band in terms of accuracy and sensitivity, particularly with respect to confirming and quantifying the Compton reflection component. This survey is meant to provide a baseline for future analysis with respect to the long-term averages for these sources and to cement the legacy of RXTE, and especially its High Energy X-ray Timing Experiment, as a contributor to AGN spectral science.
\end{abstract}

Subject headings: galaxies: active - X-rays: galaxies $-\mathrm{X}$-rays: spectra

\section{INTRODUCTION}

$\mathrm{X}$-ray emission is seen universally in active galactic nuclei (AGN) and offers a wealth of information about the geometrical and physical make-up of the accreting supermassive black holes at the hearts of distant galaxies. The primary X-ray continuum is thought to arise from the hot, Comptonizing electron or electron-positron pair corona close to the black hole (Haardt et al. 1994). In many galaxies and especially in Seyferts the continuum is reprocessed by the accretion disk or other circumnuclear material creating commonly seen reflection features: the $\mathrm{Fe} \mathrm{K}$ emission around $6-7 \mathrm{keV}$ and the so-called Compton reflection hump peaking around $20-30 \mathrm{keV}$. Nandra \& Pounds (1994) performed a spectral survey of 27 Seyferts observed with Ginga in the $1.5-37 \mathrm{keV}$ range and confirmed that Fe lines and Compton reflection humps were indeed common. Further work using the high resolution of the Advanced Satellite for Cosmology and Astrophysics (ASCA), XMMNewton and Chandra in the $2-10 \mathrm{keV}$ range by Reynolds (1997), Yaqoob \& Padmanabhan (2004), Nandra (2006), and Nandra et al. (2007) has yielded detailed Fe K complex parameters. However each of these instruments lacks broadband coverage above about $10 \mathrm{keV}$, which complicates determination of the underlying continuum.

In the last decade the $20-100 \mathrm{keV}$ sky has become more accessible, a development which is especially interesting for AGN because 20-100 keV AGN fluxes do not suffer from absorption by gas along the line of sight to the nucleus. INTEGRAL and Swift-BAT surveys are turning up large numbers of obscured AGN (including some that are rather bright above $20 \mathrm{keV}$ ) which are difficult to study in lower energy bands (e.g. Winter et al. 2009, Bird et al. 2007). These surveys yield detections and fluxes, and constrain AGN number densities and luminosity functions. However, for energy spectra in this band, the community has relied mainly on Rossi X-ray Timing Explorer (RXTE), BeppoSAX and Suzaku, and less recently, High Energy Astronomy Observatory-1 (HEAO-1)

\footnotetext{
erivers@ucsd.edu

${ }^{1}$ University of California, San Diego, Center for Astrophysics and Space Sciences, 9500 Gilman Dr., La Jolla, CA 92093-0424, USA
}

and the Compton Gamma Ray Observatory's Oriented Scintillation Spectrometer Experiment (CGRO-OSSE). Of these, $R X T E$, launched at the end of 1995, is the longest currently running X-ray mission and the public archive contains over 14 years' worth of data.

In this paper, we have taken advantage of the large quantity of AGN data and RXTE's broad bandpass to investigate the form of AGN X-ray spectral data. We concentrate on quantifying the strengths of Compton reflection components which will tighten constraints on the quantities of Compton-thick circumnuclear gas, and searching for cutoffs or rollovers in the highenergy power-law continuum, which could place constraints on thermal Comptonization processes in the corona (Haardt et al. 1994). We have closely examined RXTE archival data for 23 of the X-ray brightest AGN and we were able to construct spectra summed over the last 14 years covering from 3 to above $100 \mathrm{keV}$. Our analysis takes advantage of the fact that the two pointed-observation instruments, the Proportional Counter Array (PCA) and the High Energy X-ray Timing Experiment (HEXTE), are always operating simultaneously, removing ambiguity due to source variability inherent in splicing non-simultaneous $<10$ and $>10 \mathrm{keV}$ data sets from different missions as is commonly done to achieve broadband coverage.

$R X T E$ has been commonly used for continuous and sustained long-term monitoring and many of the X-ray brightest AGN have been monitored for at least 5-10 years. For many of the objects in our sample, the spectra are time-averaged over timescales of years, and thus this paper serves as a reference for long-term average spectral properties in the hard and very hard X-ray bands. For these sources, this complements the information obtained from individual "long-look" spectra, as are commonly obtained with many other X-ray observatories for which monitoring is difficult (e.g., XMM-Newton, Chandra). This investigation also serves to support the legacy of RXTE by maximizing the AGN science return from HEXTE.

This paper is structured in the following way: Section 2 describes the archive, instruments, and data reduction process. Section 3 details the methods and analysis. Section 4 discusses our results, including the Compton reflection hump and the high-energy rollover. Section 5 contains a summary of the main results of the paper. We have included 
TABLE 1

TARGET LIST

\begin{tabular}{|c|c|c|c|c|c|c|}
\hline Source Name & Type & $\overline{z z}$ & $\begin{array}{l}\text { Galactic } N_{\mathrm{H}}{ }^{1} \\
\left(10^{20} \mathrm{~cm}^{-2}\right)\end{array}$ & $\begin{array}{l}\text { PCA } \\
\text { Expo }^{2}(\mathrm{ks})\end{array}$ & $\begin{array}{l}\text { HEXTE-A, B } \\
\text { Expo }^{2}(\mathrm{ks}, \mathrm{ks})\end{array}$ & Lightcurve References \\
\hline \multicolumn{7}{|c|}{ Type 1 Seyfert Galaxies } \\
\hline NGC 4151 & Sy1.5 & 0.003319 & 2.53 & 536 & $180, \quad 180$ & Markowitz et al. (2003b) \\
\hline NGC 3783 & Sy1 & 0.009730 & 10.3 & 1297 & $204, \quad 365$ & Arévalo et al. (2009), Summons et al. (2007) \\
\hline NGC 5548 & Sy1.5 & 0.017175 & 1.52 & 927 & $294, \quad 312$ & Markowitz et al. (2003b), Uttley et al. (2003) \\
\hline NGC 3516 & Sy1.5 & 0.008836 & 3.12 & 947 & $293, \quad 292$ & Markowitz et al. (2003b), Maoz et al. (2002) \\
\hline NGC 3227 & Sy1.5 & 0.003859 & 1.84 & 1050 & $283, \quad 284$ & Uttley \& $\mathrm{M}^{\mathrm{c}}$ Hardy (2005) \\
\hline NGC 4593 & Sy1 & 0.009000 & 1.55 & 960 & $168, \quad 282$ & Summons et al., in prep., Markowitz \& Reeves (2009) \\
\hline NGC 7469 & Sy1.2 & 0.016317 & 4.72 & 1065 & $243, \quad 305$ & Markowitz (2010), Nandra \& Papadakis (2001) \\
\hline \multicolumn{7}{|c|}{ Radio-Loud Seyfert 1s } \\
\hline 3C 111 & BLRG & 0.048500 & $119.1^{3}$ & 808 & $127, \quad 238$ & Chatterjee et al. (2011), Markowitz \& Edelson (2004) \\
\hline Cen A & NLRG & 0.001825 & 9.07 & 632 & $110, \quad 200$ & Rothschild et al. (2011), Rothschild et al. (2006) \\
\hline NGC 5506 & Sy1.9 & 0.006181 & 4.64 & 698 & $202, \quad 201$ & Uttley \& $\mathrm{M}^{\mathrm{c}}$ Hardy (2005) \\
\hline MCG-5-23-16 & Sy2 & 0.008486 & 8.28 & 180 & $55, \quad 55$ & this work \\
\hline NGC 4507 & Sy2 & 0.011801 & 7.04 & 145 & $47, \quad 47$ & this work \\
\hline \multicolumn{7}{|l|}{ Compton-thick } \\
\hline Circinus & Sy2 & 0.001448 & 50.4 & 97 & $33, \quad 32$ & this work \\
\hline NGC 7582 & Sy2 & 0.005254 & 1.23 & 185 & $43, \quad 43$ & this work \\
\hline NGC 4945 & Sy2 & 0.001878 & 13.9 & 999 & $222, \quad 320$ & Mueller et al. (2004) \\
\hline \multicolumn{7}{|l|}{ Blazars } \\
\hline $3 C 273$ & FSRQ & 0.158339 & 1.62 & 1810 & $430, \quad 530$ & Kataoka et al. (2000) \\
\hline 3C 454.3 & FSRQ & 0.859000 & 7.24 & 37 & $13, \quad 13$ & Jorstad et al. (2010) \\
\hline
\end{tabular}

NotE. - The 23 targets selected for our sample due to reasonably bright hard (20-100 keV) X-ray spectra and sufficient exposure time to achieve good statistics with HEXTE. Targets are listed by $2-10 \mathrm{keV}$ flux within each object class (flux values given in Table 8 .

1 Values taken from the LAB survey (Kalbera et al. 2005).

2 Good exposure time after screening.

$373 \%$ of the $N_{\mathrm{H}}$ column for $3 \mathrm{C} 111$ is from a molecular cloud along the line of sight.

notes on individual sources with details of modeling and comparisons to previous work unique to each source in Appendix A.

\section{ARCHIVAL OBSERVATIONS AND DATA REDUCTION}

\subsection{The Archive}

$R X T E$ includes two pointed observation instruments, the PCA (Jahoda et al. 2006) and HEXTE (Rothschild et al. 1998). The PCA is usually the primary instrument when observing; HEXTE is not as sensitive as the PCA below $20 \mathrm{keV}$ and is commonly overlooked for faint sources such as AGN, especially for short observations. This investigation demonstrates that accumulation of data over long timescales can indeed yield high quality PCA + HEXTE spectra for the brightest AGN.

The sampling of the AGN in the RXTE public archive is highly inhomogeneous, as targets were proposed by various groups for a variety of reasons. This includes, for example, multi-timescale, continuous monitoring of Seyferts for power spectral density (PSD) measurements, long-look style observations for tens of ks at a time, and target of opportunity observations on flaring blazars as part of multiwavelength campaigns. For each target, we simply used all data available in the public archive regardless of sampling. We used data up to 2008 July (plus proprietary data up to 2009 July for NGC 7469 and proprietary data in 2009 January and February for Cen A). Light curves in Figures 13 show the distribution of observations over the past 14 years.

We did not make any effort to isolate a particular class of AGN.
Target selection was guided by HEXTE data quality alone. Our goal was to obtain high-quality spectra out to at least $100 \mathrm{keV}$ for each case. This required the combination of the source being sufficiently $\mathrm{X}$-ray bright in the $20-100 \mathrm{keV}$ range and having been observed with a sufficiently long exposure time. The limiting 20-100 keV flux was $\gtrsim 5 \times 10^{-11} \mathrm{erg} \mathrm{cm}^{-2} \mathrm{~s}^{-1}$ depending on exposure time and the longterm trends in the HEXTE background; see Section 2.4 for details.

These criteria for target selection bias our sample towards objects with flat spectra except in the case of objects which happen to have high fluxes and/or a large amount of exposure time. Hence this sample is not statistically complete, for example we did not have any Narrow Line Seyfert 1's in our sample since they tend to be faint and have steeper spectra than typical Broad Line Seyfert 1's (Grupe et al. 1999, Vaughan et al. 2001, Xu et al. 2003) and none which were observed by $R X T E$ with sufficient time to obtain a $3 \sigma$ detection at $100 \mathrm{keV}$. The final sample of 23 AGN contains a variety of AGN subclasses, including 17 Seyferts, two broad-line radio galaxies (BLRGs), two flat-spectrum radio quasars (FSRQs), and two BL Lac objects. Some objects have PCA + HEXTE spectra already published, but in most cases we update these spectra by including additional data.

Table 1 lists the source name, AGN type, redshift $(z)$, and the Galactic column density $N_{\mathrm{H}, \text { Gal }}$, as well as the total exposure time for each of the RXTE instruments. Lightcurve references are also included.

For the purposes of analysis, we have most-often broken our sample down into four categories: Seyfert 1's, Compton-thick Seyfert 2's, Compton-thin Seyfert 2's, and blazars. Note that since Seyfert 1's and 

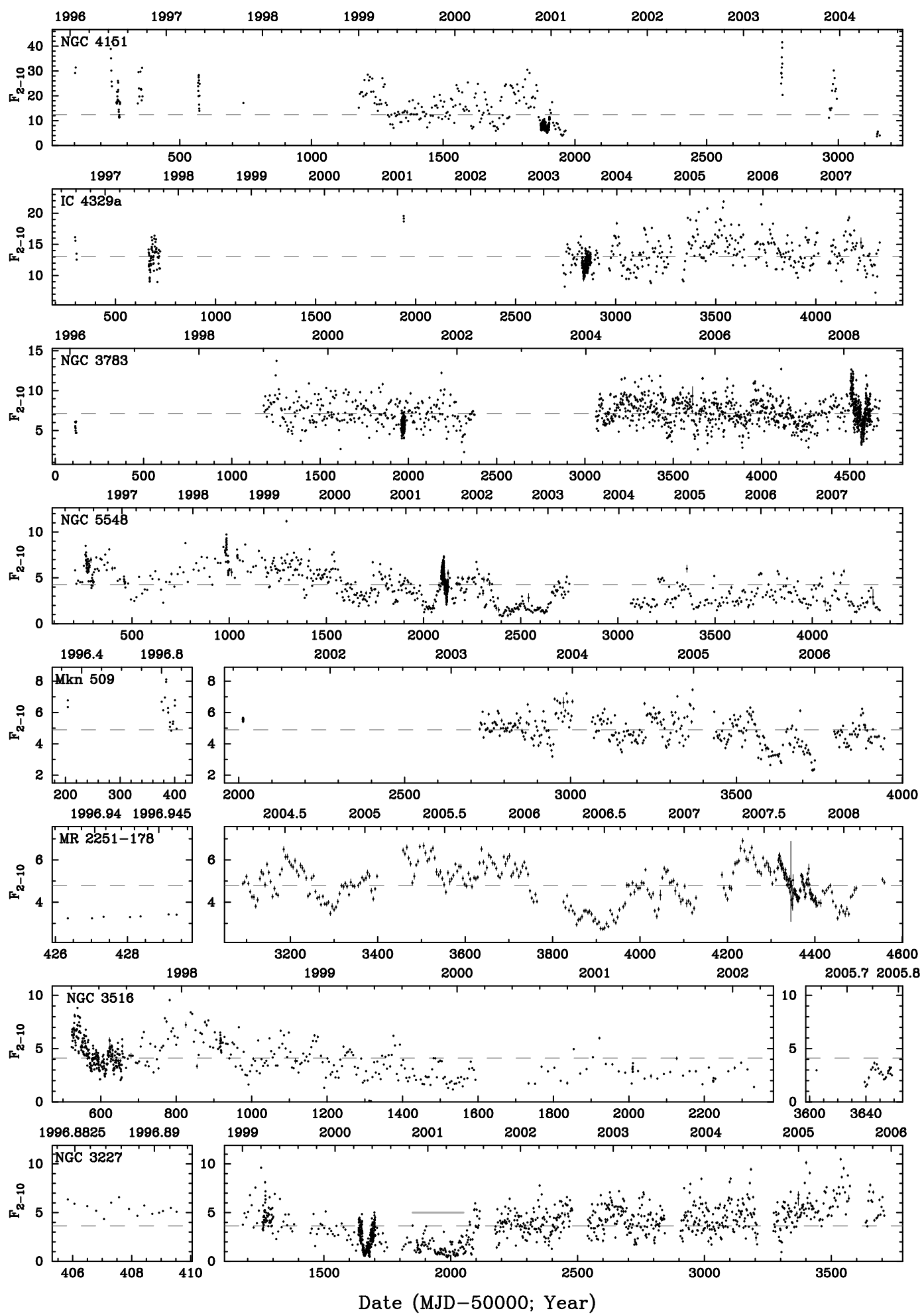

FIG. 1.- 2-10 keV PCA flux light curves for the targets in our sample, with one flux point for each observation $\left(F_{2-10}\right.$ in units of erg $\left.\mathrm{cm}^{-2} \mathrm{~s}^{-1}\right)$. In many cases, error bars are smaller than the size of the data point. Dashed lines indicate the average long-term flux for each object.

2's are classified optically, there is not always a firm correspondence between the levels of X-ray absorption along the line of sight and the classification. Seyfert 1's include Seyfert 1-1.5's while Seyfert 2's include Seyfert 1.8-2's. The distinction between Compton-thick and Compton-thin Seyfert 2's is important to us since the Compton-thick sources tend to have much more complicated X-ray spectra potentially causing model degeneracy in fitting. More details can be found in the notes on each Compton-thick source. Since 3C 273 has X-ray spectral properties more closely resembling a BLRG than a typical blazar (displays an Fe line and a very weak Compton hump), it is included with these solely for the purpose of spectral analysis in this paper.

\subsection{Proportional Counter Array (PCA) Data Reduction}

For all PCA and HEXTE data extraction and analysis we used HEASOFT version 6.7 software. The PCA consists of five largearea, collimated proportional counter units (PCUs). Reduction of the PCA data followed standard extraction and screening procedures. PCA STANDARD-2 data were collected from PCU's 0, 1 and 2 prior to 1998 December 23; PCU's 0 and 2 from 1998 December 23 until 2000 May 12; and PCU 2 only after 2000 May 12. PCU 0 lost its propane veto layer starting on 2000 May 12, PCUs 1, 3, and 4 have been known to suffer from repeated breakdown during on-source time, and PCU 1 lost its propane layer on 2006 December 25. In addition, PCU 2 is the best calibrated of the PCU's and has maintained 

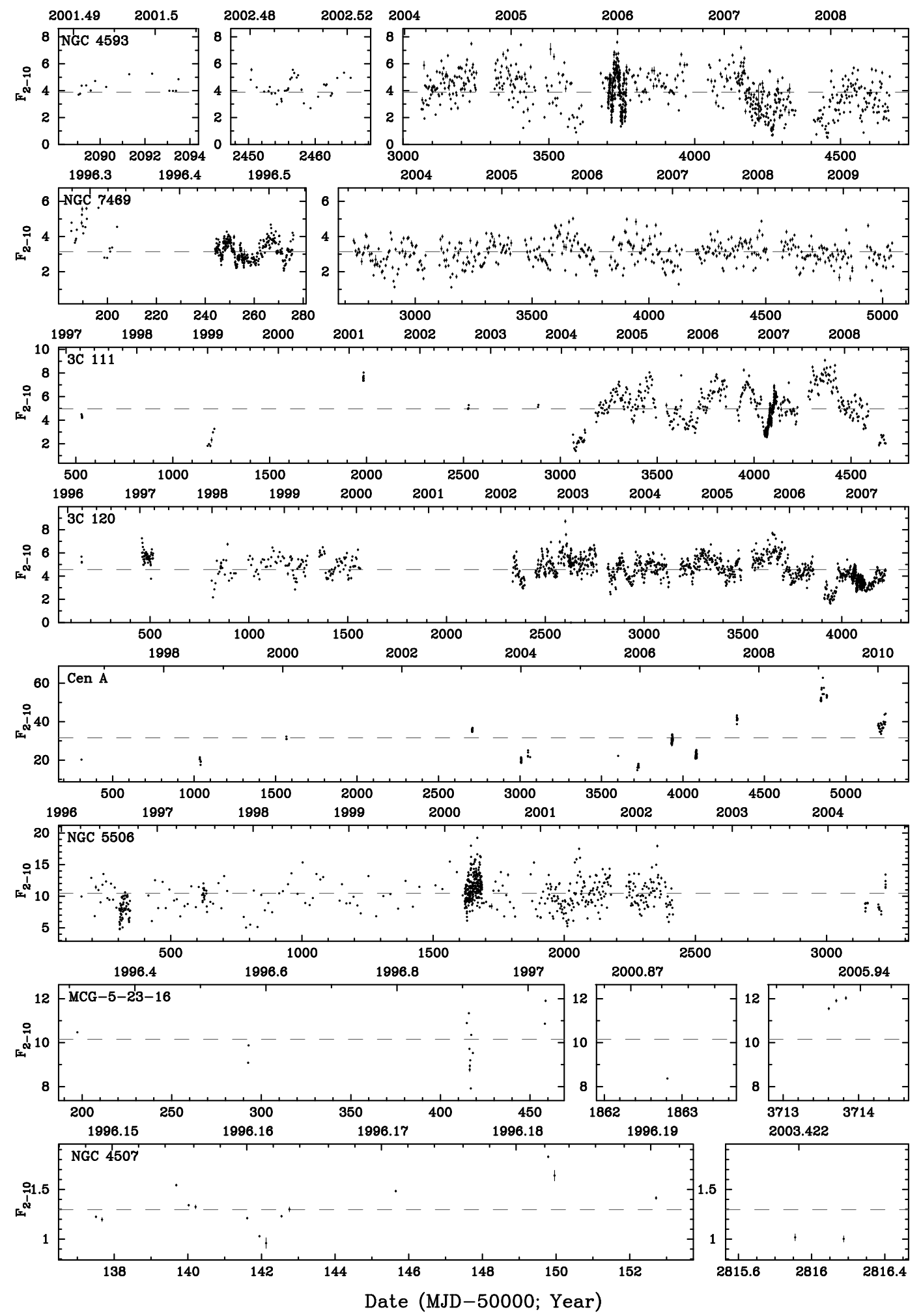

FIG. 2.- 2-10 keV PCA flux light curves for the targets in our sample, with one flux point for each observation $\left(F_{2-10}\right.$ in units of erg $\left.\mathrm{cm}^{-2} \mathrm{~s}^{-1}\right)$. In many cases, error bars are smaller than the size of the data point. Dashed lines indicate the average long-term flux for each object.

consistent functionality for the duration of RXTE's mission (Jahoda et al. 2006).

To maximize signal-to-noise ratios we extract events from the top Xe layer of the PCA only. Data were rejected if they were gathered less than $10^{\circ}$ from Earth's limb, within 20 minutes of satellite passage through the South Atlantic Anomaly (SAA), if the satellite's pointing offset was greater than 0.01 , or if the ELECTRON2 value (a measure of particle flux) was $>0.1$. As the PCA is a non-imaging instrument, the background was estimated using the "L7-240" background models, appropriate for faint sources when the total count rate was below 40 counts s ${ }^{-1} \mathrm{PCU}^{-1}$; see e.g., Edelson \& Nandra (1999) for details on PCA background subtraction, the dominant source of systematic uncertainty (e.g., in total broadband count rate) in these data. Some observations of Cen A and Mkn 421 fell above this flux threshold, and we used the Sky-VLE background model on those observations.

Observations of $3 \mathrm{C} 454.3$ were obtained using a pointing position 0.52 to the southeast of $3 \mathrm{C} 454.3$ to completely eliminate contributions to the spectrum from the cataclysmic variable, IM Peg, located about 0.72 to the northwest of 3C 454.3 (see Jorstad et al. 2010). At this offset position, the PCA and HEXTE collimator efficiencies are both $45 \%$ (e.g., Jahoda et al. 2006); we corrected for this when generating the response matrices (below) and during spectral fitting. 



FIG. 3.- 2-10 keV PCA flux light curves for the targets in our sample, with one flux point for each observation $\left(F_{2-10}\right.$ in units of erg $\left.\mathrm{cm}^{-2} \mathrm{~s}^{-1}\right)$. In many cases, error bars are smaller than the size of the data point. Dashed lines indicate the average long-term flux for each object. For NGC 7582 data were ignored between MJD 51,850-52,050, see text.

Source and background spectra were extracted for each on-source ID, and added using SUMPHA to create time-average source and background spectra. Source spectra were binned to a minimum of 50 counts $\operatorname{bin}^{-1}$ to ensure use of the $\chi^{2}$ statistic, although for nearly all energy bins, the systematic uncertainties dominate over the statistical uncertainties. A systematic uncertainty of $0.5 \%$ was added to the source PCA spectra. As the response of the PCA slowly hardens slightly over time due to the gradual leak of xenon gas into the propane layer in each $\mathrm{PCU}$, response files were generated for each separate observation ID (ObsID) using PCARMF version 11.7 and the latest calibration files available as of 2009 October. A time-averaged response file was generated by summing the individual response files and weighting them by their good exposure time and number of active PCU's.

\subsection{High Energy X-ray Timing Experiment (HEXTE) Data Reduction}

HEXTE consists of two independent clusters (A and B), each containing four $\mathrm{NaI}(\mathrm{Tl}) / \mathrm{CsI}(\mathrm{Na})$ phoswich scintillation counters (Rothschild et al. 1998) which share a common $1^{\circ}$ FWHM field of view. Each of the eight detectors has a net open area of about $200 \mathrm{~cm}^{2}$. Source and background spectra were extracted from each individual $R X T E$ visit using Science Event data and standard extraction procedures. Data were rejected if they were gathered less than $10^{\circ}$ from Earth's limb, within 30 minutes of satellite passage through the SAA, or if the satellite's pointing offset was $>0.01$.

To measure real-time background measurements, the two HEXTE clusters each undergo two-sided rocking to offset positions $1.5 \mathrm{off}$ source, with default switching every 16 s before 1998 January 8 and every $32 \mathrm{~s}$ thereafter. This allows for background subtraction for any timescale from $32 \mathrm{~s}$ up to the lifetime of the mission.

We used the online resources HEXTErock and HEASARC's

${ }^{2}$ http://mamacass.ucsd.edu/cgi-bin/HEXTErock.html 
TABLE 2

20-100 KEV BAND

\begin{tabular}{|c|c|c|c|c|c|}
\hline$\overline{\text { Source }}$ & Photon Index & $\begin{array}{c}\text { HEXTE-A Flux } \\
\left(10^{-10} \mathrm{erg} \mathrm{s}^{-1}\right)\end{array}$ & $\begin{array}{c}\text { HEXTE-B Flux } \\
\left(10^{-10} \mathrm{erg} \mathrm{s}^{-1}\right)\end{array}$ & $\begin{array}{c}\text { \% of Background } \\
\text { A, B }\end{array}$ & $\overline{\chi^{2} / \mathrm{dof}}$ \\
\hline NGC 4151 & $1.84 \pm 0.02$ & 4.45 & 4.58 & $6.2, \quad 6.4$ & $536 / 53$ \\
\hline IC 4329A & $1.89 \pm 0.04$ & 1.91 & 1.92 & $4.0, \quad 4.5$ & $140 / 49$ \\
\hline NGC 3783 & $1.82 \pm 0.05$ & 1.19 & 1.29 & $1.9, \quad 2.2$ & $90 / 43$ \\
\hline NGC 5548 & $1.79 \pm 0.08$ & 0.78 & 0.79 & $1.1, \quad 1.1$ & $53 / 33$ \\
\hline Mkn 509 & $1.87 \pm 0.09$ & 0.80 & 0.73 & $1.4, \quad 1.5$ & $51 / 37$ \\
\hline MR 2251-178 & $1.8 \pm 0.2$ & 0.70 & 0.71 & $0.9, \quad 1.0$ & $20 / 23$ \\
\hline NGC 3516 & $1.8 \pm 0.1$ & 0.89 & 0.91 & $1.1, \quad 1.2$ & $51 / 29$ \\
\hline NGC 3227 & $1.77 \pm 0.08$ & 0.76 & 0.76 & $1.2, \quad 1.4$ & $53 / 39$ \\
\hline NGC 4593 & $1.76 \pm 0.09$ & 0.70 & 0.66 & $1.4, \quad 1.4$ & $52 / 37$ \\
\hline NGC 7469 & $1.8 \pm 0.2$ & 0.47 & 0.51 & $0.7, \quad 0.8$ & $29 / 31$ \\
\hline 3C 111 & $1.8 \pm 0.1$ & 0.82 & 0.81 & $1.6, \quad 1.6$ & $48 / 47$ \\
\hline $3 \mathrm{C} 120$ & $1.78 \pm 0.06$ & 0.80 & 0.75 & $1.3, \quad 1.4$ & $62 / 47$ \\
\hline Cen A & $1.78 \pm 0.01$ & 6.02 & 7.38 & $9.9, \quad 13.7$ & $62 / 53$ \\
\hline NGC 5506 & $1.87 \pm 0.04$ & 1.75 & 1.74 & $2.2, \quad 2.3$ & $194 / 37$ \\
\hline MCG-5-23-16 & $1.9 \pm 0.1$ & 1.48 & 1.52 & $1.9, \quad 1.9$ & $56 / 35$ \\
\hline NGC 4507 & $1.6 \pm 0.1$ & 1.33 & 1.73 & $1.7, \quad 1.7$ & $40 / 35$ \\
\hline Circinus & $1.72 \pm 0.07$ & 2.02 & 2.16 & $2.6, \quad 2.9$ & $259 / 35$ \\
\hline NGC 7582 & $1.8 \pm 0.3$ & 0.58 & 0.60 & $1.5, \quad 1.5$ & $30 / 13$ \\
\hline NGC 4945 & $1.32 \pm 0.03$ & 1.77 & 1.80 & $3.4, \quad 3.9$ & $894 / 53$ \\
\hline $3 \mathrm{C} 273$ & $1.74 \pm 0.02$ & 1.80 & 1.98 & $2.9, \quad 3.5$ & $79 / 53$ \\
\hline $3 \mathrm{C} 454.3$ & $1.5 \pm 0.3$ & 0.64 & 0.46 & $1.7, \quad 1.4$ & $9 / 20$ \\
\hline Mkn 421 & $2.70 \pm 0.04$ & 1.11 & 1.04 & $2.3, \quad 2.2$ & $47 / 41$ \\
\hline 1ES $1959+650$ & $2.32 \pm 0.08$ & 0.78 & 0.81 & $1.9, \quad 2.2$ & $47 / 45$ \\
\hline
\end{tabular}

NOTE. - These are results for simple power-law fits to the $20-100 \mathrm{keV}$ band only using data from both HEXTE clusters. $\Gamma$ is tied between HEXTE-A and -B for all objects.

skyview too (looking at $S$ wift-BAT and $R X T E$ all-sky slew survey images) to ensure that there were no possible contaminating bright sources above $20 \mathrm{keV}$ within $2^{\circ}$ of each source.

Cluster A data taken during the following times were excluded, as the cluster did not rock on/off-source: 2004 December 13 - 2005 January 14, 2005 December 12 - 2006 January 4, during 2006 January 25, and after 2006 March 14. Detector 2 in cluster B lost spectral capabilities in 1996; spectra were derived from the other three detectors only. Deadtime corrections were applied to account for cluster rocking, pulse analyzer electronics, and the recovery time following scintillation pulses caused by high-energy charged particles; typically, the HEXTE deadtime is around $30 \%-40 \%$.

We did not combine cluster $\mathrm{A}$ and $\mathrm{B}$ data; for observations after spring of 2006 we used only cluster B data and many of our sources have data from this period. We used standard response matrices, hexte_00may26_pwa.arf, hexte_97mar20c_pwa.rmf, hexte_00may26_pwb013.arf, and hexte_97mar20c_pwb013.rmf.

\subsection{HEXTE Background Considerations}

The HEXTE background is sufficiently bright that it dominates over the source spectra for typical AGN fluxes. The HEXTE background subtraction is well-understood for source fluxes down to approximately $1 \%$ of the background (Gruber et al. 1996). Sources with $20-100 \mathrm{keV}$ fluxes fainter than approximately $1 \%$ of the background and/or with insufficient exposure time therefore had very large error bars in their net spectra near 30 and $70 \mathrm{keV}$, the energies of the strongest activation lines that comprise the HEXTE background, or were not detected out to $100 \mathrm{keV}$. For sources whose net fluxes were in the range $0.7 \%-1.2 \%, 1.2 \%-1.5 \%$, and $1.5 \%-2.0 \%$ of the background, the good exposure times per HEXTE cluster after screening required to yield acceptable-quality spectra were approximately 250 , 100 , and $50 \mathrm{ks}$, respectively.

The average HEXTE background rate has dropped by 50\% (from $\sim 12$ to $6 \times 10^{-9} \mathrm{erg} \mathrm{cm}^{-2} \mathrm{~s}^{-1}$ ) over a decade as the altitude of the $R X T E$ spacecraft has decreased, thus intercepting smaller regions of

\footnotetext{
${ }^{3}$ http://skyview.gsfc.nasa.gov/
}

the SAA (Fürst et al. 2009), resulting in a smaller particle flux through the detectors. The reader is referred to Appendix A for further details of the composition and long-term variability of the HEXTE background.

We combined $16 \mathrm{~s}$ and $32 \mathrm{~s}$ rocking within each cluster, however this proved to be non-trivial because SUMPHA does not take into account the difference in background exposure time relative to onsource time which is $3 / 4$ for $16 \mathrm{~s}$ and $7 / 8$ for $32 \mathrm{~s}$. To eliminate this problem we performed background subtraction on the $16 \mathrm{~s}$ and 32 $\mathrm{s}$ separately before summing the spectra. We also created summed background files to use as correction files for fitting (see Section 3 below).

\section{METHODS AND ANALYSIS}

All spectral fitting below was done with XSPEC version $12.5 .1 \mathrm{k}$ utilizing the abundances of Wilms et al. (2000) and cross-sections of Verner et al. (1996). Unless otherwise stated, uncertainties given are $90 \%$ confidence $\left(\Delta \chi^{2}=2.71\right.$ for one interesting parameter).

We aimed to truncate each source spectrum at an energy where our signal-to-noise ratio dropped below $3 \sigma$. This energy is different for each source. We also made use of the "RECORN" component in XSPEC to correct for imperfections in the background subtraction (PCA) and dead time estimation as a function of count rate (PCA and HEXTE). Typical values were around $1 \%$ of the background for the PCA and $0.16 \%$ for the HEXTE clusters. Additionally, free crossnormalization factors for HEXTE-A and -B with respect to the PCA had average values of 0.79 and 0.81 respectively.

\subsection{The 20-100 keV Bandpass}

To explore target properties in the $20-100 \mathrm{keV}$ bandpass we fit a simple power law to HEXTE-only data. We fit HEXTE-A and B spectra with the power-law normalizations untied due to the fact that for several sources, the HEXTE-B data covered a longer time span than HEXTE-A. We kept the photon indices tied between A and B since untying the photon indices did not yield a significant improvement in the fit for most sources. The only exception to this was 3C 273, for which untying the photon indices and power law normalizations led 
TABLE 3

BASELINE MODEL FITS

\begin{tabular}{|c|c|c|c|c|c|c|c|c|c|}
\hline Source & $\overline{\bar{\Gamma}}$ & $\begin{array}{c}A^{1} \\
\left(10^{-2}\right)\end{array}$ & $\begin{array}{c}N_{\mathrm{H}} \\
\left(10^{22} \mathrm{~cm}^{-2}\right)\end{array}$ & $\begin{array}{c}\text { Partial Covering } \\
N_{\mathrm{H}}\left(10^{22} \mathrm{~cm}^{-2}\right)\end{array}$ & $\begin{array}{c}\text { Covering } \\
\text { Frac. }\left(f_{\text {cov }}\right)\end{array}$ & $\begin{array}{c}\text { Fe Line } \\
\text { Energy }(\mathrm{keV})\end{array}$ & $\begin{array}{c}\sigma \\
(\mathrm{keV})\end{array}$ & $\begin{array}{c}I_{\mathrm{Fe}}\left(10^{-4}\right) \\
\left(\mathrm{ph} \mathrm{cm}^{-2} \mathrm{~s}^{-1}\right)\end{array}$ & $\overline{\chi^{2} / \mathrm{dof}}$ \\
\hline NGC 4151 & $1.84 \pm 0.02$ & $13.3 \pm 0.1$ & 3.4(fixed) & $97 \pm 2$ & $0.66 \pm 0.04$ & $6.06 \pm 0.05$ & $0.9 \pm 0.1$ & $12.0 \pm 0.6$ & $682 / 84$ \\
\hline IC $4329 A$ & $1.75 \pm 0.01$ & $3.84 \pm 0.05$ & & & & $6.25 \pm 0.09$ & $<0.5$ & $1.8 \pm 0.2$ & $381 / 81$ \\
\hline NGC 3783 & $1.66 \pm 0.03$ & $2.0 \pm 0.1$ & & & & $6.3 \pm 0.2$ & $0.3 \pm 0.1$ & $1.7 \pm 0.9$ & $242 / 71$ \\
\hline NGC 5548 & $1.67 \pm 0.01$ & $1.40 \pm 0.04$ & & & & $6.1 \pm 0.2$ & $0.5 \pm 0.4$ & $1.0 \pm 0.3$ & $102 / 59$ \\
\hline Mkn 509 & $1.70 \pm 0.01$ & $1.40 \pm 0.03$ & & & & $6.2 \pm 0.8$ & $<0.8$ & $0.7 \pm 0.1$ & $92.8 / 63$ \\
\hline MR 2251-178 & $1.63 \pm 0.02$ & $0.97 \pm 0.05$ & & & & $6.3 \pm 0.4$ & $0.9 \pm 0.6$ & $0.9 \pm 0.3$ & $75.9 / 49$ \\
\hline NGC 3516 & $1.72 \pm 0.03$ & $1.5 \pm 0.2$ & & & $0.55^{2} \pm 0.10$ & $6.23 \pm 0.06$ & $<0.3$ & $0.9 \pm 0.1$ & $122 / 61$ \\
\hline NGC 3227 & $1.60 \pm 0.01$ & $0.89 \pm 0.03$ & $0.3 \pm 0.3$ & & & $6.2 \pm 0.2$ & $0.4 \pm 0.3$ & $0.9 \pm 0.2$ & $117 / 70$ \\
\hline NGC 4593 & $1.77 \pm 0.01$ & $1.12 \pm 0.03$ & & & & $6.36 \pm 0.06$ & $<0.4$ & $1.0 \pm 0.1$ & $118 / 60$ \\
\hline NGC 7469 & $1.78 \pm 0.02$ & $0.89 \pm 0.03$ & & & & $6.27 \pm 0.09$ & $<0.5$ & $0.6 \pm 0.2$ & $96.7 / 54$ \\
\hline 3C 111 & $1.67 \pm 0.02$ & $1.24 \pm 0.05$ & & & & $6.2 \pm 0.2$ & $0.7 \pm 0.3$ & $1.2 \pm 0.4$ & $102 / 73$ \\
\hline $3 \mathrm{C} 120$ & $1.68 \pm 0.03$ & $1.18 \pm 0.07$ & & & & $6.3 \pm 0.3$ & $<0.8$ & $1.2 \pm 0.5$ & $97.5 / 77$ \\
\hline $3 \mathrm{C} 273$ & $1.66 \pm 0.01$ & $2.71 \pm 0.02$ & & & & $7.0_{-0.8}^{+*}$ & $<1.0$ & $0.4 \pm 0.2$ & $158 / 83$ \\
\hline Cen A & $1.83 \pm 0.01$ & $16.0 \pm 0.2$ & $16.9 \pm 0.3$ & & & $6.38 \pm 0.09$ & $<0.4$ & $4.9 \pm 0.7$ & $143 / 84$ \\
\hline NGC 5506 & $1.62 \pm 0.02$ & $2.2 \pm 0.1$ & $<1.0$ & & & $5.8 \pm 0.1$ & $0.9 \pm 0.1$ & $6.6 \pm 1.0$ & $527 / 70$ \\
\hline MCG-5-23-16 & $1.67 \pm 0.02$ & $2.5 \pm 0.1$ & $1.6 \pm 0.4$ & & & $6.26 \pm 0.06$ & $0.4 \pm 0.1$ & $2.5 \pm 0.2$ & $135 / 68$ \\
\hline NGC 4507 & $1.62 \pm 0.06$ & $1.4 \pm 0.2$ & $85 \pm 2$ & & & $6.4 \pm 0.5$ & $<0.8$ & $1.7 \pm 0.2$ & $148 / 71$ \\
\hline NGC 7582 & $1.12 \pm 0.08$ & $0.13 \pm 0.02$ & $11 \pm 2$ & & & $6.2 \pm 0.8$ & $<0.8$ & $0.8 \pm 0.1$ & $179 / 34$ \\
\hline \multirow[t]{2}{*}{ NGC 7582-PC ${ }^{3}$} & $1.8 \pm 0.1$ & $1.8_{-0.7}^{+1.2}$ & $24 \pm 4$ & $360 \pm 60$ & $0.6_{-0.3}^{+0.6}$ & $6.2 \pm 0.1$ & $<0.5$ & $0.7 \pm 0.1$ & $40 / 32$ \\
\hline & & & & $\Gamma_{\text {SXPL }}{ }^{4}$ & $A_{\mathrm{SXPL}}{ }^{1}\left(10^{-2}\right)$ & & & & \\
\hline Circinus & $1.62 \pm 0.03$ & $2.8 \pm 0.3$ & $395 \pm 9$ & $1.62 \pm 0.03$ & $0.41 \pm 0.02$ & $6.43 \pm 0.01$ & $0.25 \pm 0.03$ & $5.8 \pm 0.1$ & $338 / 64$ \\
\hline NGC 4945 & $1.73 \pm 0.04$ & $3.53 \pm 0.03$ & $600 \pm 20$ & $1.8 \pm 0.1$ & $0.15 \pm 0.03$ & $6.45 \pm 0.07$ & $<0.4$ & $0.65 \pm 0.07$ & $196 / 82$ \\
\hline
\end{tabular}

NOTE. - Best fit model parameters for our baseline model fit (no Compton hump or high-energy rollover modeled). $N_{\mathrm{H}}$ is the full-covering cold column in excess of $N_{\mathrm{H}, \mathrm{Gal}}$. Source name in bold indicates that we conclude this model is the best description of the spectrum.

${ }^{1}$ Power law normalization ( $\mathrm{ph} \mathrm{keV}^{-1} \mathrm{~cm}^{-2} \mathrm{~s}^{-1}$ at $1 \mathrm{keV}$ )

${ }^{2}$ Partial covering warm absorber with $\log (\xi)$ fixed at 2.19 and column density fixed at $2 \times 10^{23} \mathrm{~cm}^{-2}$ but covering fraction left as a free parameter.

${ }^{3}$ Alternative model with partial covering Compton-thick absorber as suggested by Turner et al. (2000). See Appendix A.19 for details.

${ }^{4}$ SXPL parameters are for the soft X-ray power law due to leaked or scattered emission.

to an improvement in fit of $\Delta \chi^{2} /$ dof $=-8 / 2$ with photon indices of $1.79 \pm 0.02$ and $1.71 \pm 0.02$ for cluster $\mathrm{A}$ and $\mathrm{B}$ respectively. Tied photon indices, absorbed fluxes, and goodness of fits are listed in Table 2 Brightness level as a percent of background in the $20-100 \mathrm{keV}$ band is also listed. Note that there are about a half-dozen sources where a simple power law is a bad fit, due to extra curvature possibly indicating a Compton reflection hump or a high-energy rollover.

\subsection{Broadband Fitting of PCA and HEXTE Combined Data}

Since Seyfert galaxies share many common spectral attributes we applied similar models to all the sources that were Seyferts or Seyfertlike, including the BLRGs and the FSRQ 3C 273 in which we find evidence for an $\mathrm{Fe}$ line and possibly even a weak Compton reflection hump, both of which are atypical of blazars. The remaining three blazars were treated separately (see Section 3.3) since they do not show many of the spectral features seen in the rest of our sample.

With the latest calibration it was not necessary to add instrumental features to the models, such as a Gaussian at $8.05 \mathrm{keV}$ for $\mathrm{Cu}$ emission or an Xe L edge near 4-5 keV, as was done by Rothschild et al. (2006) for RXTE observations of Cen A.

The Fe K line is virtually ubiquitous in Seyfert spectra. The PCA cannot deconvolve broad and narrow $\mathrm{Fe}$ lines, nor $\mathrm{Fe} \mathrm{K} \alpha$ and $\mathrm{K} \beta$ lines. In addition, the PCA cannot resolve an Fe line except in cases of extreme relativistic broadening. We therefore use a single Gaussian to fit the $\mathrm{Fe} \mathrm{K}$ emission complex. The reader is referred to results from instruments with superior energy resolution at $6 \mathrm{keV}$, namely XMM-Newton, Chandra-HETGS, or Suzaku, to determine if the Fe line we model is dominated by a broad or a narrow Fe component, or a blend of both in each object. For example, the presence of both broad and narrow lines has been confirmed with XMM-Newton or Suzaku for MCG-5-23-16 (Reeves et al. 2007), NGC 3516 (Markowitz et al. 2008), and 3C 120 (Kataoka et al. 2007), while broad lines have not been confirmed in Cen A (Markowitz et al. 2007) and NGC 5548 (Liu et al. 2010). In other objects, the Fe line is moderately broadened (e.g., NGC 4593, $v_{\text {FWHM }} \sim 10000 \mathrm{~km} \mathrm{~s}^{-1}$; Brenneman et al. 2007).

One of our primary goals was to make use of the $3-100 \mathrm{keV}$ spectra to test for the presence of Compton humps and high-energy cut-offs. To begin, we created a baseline model consisting of a primary power law with Galactic absorption plus an Fe line but with no Compton reflection or high-energy cutoff of the continuum. This alone was a suitable baseline for most of the Seyfert 1's. In the case of both Compton-thin and Compton-thick Seyfert 2s, the baseline model additionally included a "ZPHABS" component to model cold absorption in excess of $N_{\mathrm{H}, \mathrm{Gal}}$. In the case of the Compton-thick Seyfert 2s, Circinus and NGC 4945, we also included a soft power-law absorbed only by $N_{\mathrm{H}, \mathrm{Gal}}$, to model e.g., scattered nuclear X-ray continuum emission, thermal emission from diffuse plasma, and/or "leaked" continuum emission in the case of a partial-covering absorber. Best-fit results are listed in Table 3 for all objects.

For each object, we included warm absorber components as needed by searching through publications relying on Chandra-HETGS and/or $X M M-N e w t o n-R G S$ data. The PCA is not highly sensitive to discrete narrow absorption lines. However, if a warm absorber phase's spectral signature was to induce continuum curvature below $5 \mathrm{keV}$ of more than $1 \%-2 \%$, then we modeled it with an XSTAR table component, keeping the parameters frozen at the column density and ionization parameter specified in the literature. See notes on individual targets for details.

There were three exceptions to the above scheme: NGC 4151 was modeled to have a cold partial coverer in the line of sight (see Appendix A.1 for details). NGC 3227 was modeled to have full-covering cold absorption in excess of the Galactic column (see Appendix A.8 for details). For NGC 7582 we obtained two good fits, a "reflectiondominated" model and a "partial-covering" model. We report both model fits in Table 3 (see Appendix A.19 for details).

Next we added a Compton reflection component using PEXRAV (Magdziarz \& Zdziarski 1995) with the photon index tied to that of the continuum, all abundances set to solar, and no high-energy rollover. 
TABLE 4

REFLECTION MODELS

\begin{tabular}{|c|c|c|c|c|c|c|c|c|c|c|}
\hline$\overline{\text { Source }}$ & $\overline{\bar{\Gamma}}$ & $\begin{array}{c}A^{1} \\
\left(10^{-2}\right)\end{array}$ & $\begin{array}{c}N_{\mathrm{H}} \\
\left(10^{22} \mathrm{~cm}^{-2}\right)\end{array}$ & $\begin{array}{c}\text { Partial Cov. } \\
N_{\mathrm{H}}\left(10^{22} \mathrm{~cm}^{-2}\right)\end{array}$ & $\begin{array}{c}\text { Covering } \\
\text { Frac. }\left(f_{\text {cov }}\right)\end{array}$ & $\begin{array}{c}\text { Fe Line } \\
\text { Energy }(\mathrm{keV})\end{array}$ & $\begin{array}{c}\sigma \\
(\mathrm{keV})\end{array}$ & 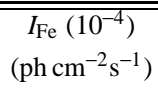 & $\overline{\bar{R}}$ & $\overline{\chi^{2} / \text { dof }}$ \\
\hline NGC 4151 & $1.90 \pm 0.02$ & $9.9 \pm 0.5$ & 3.4(fixed) & $37 \pm 3$ & $0.66 \pm 0.06$ & $6.4 \pm 0.1$ & $<0.5$ & $2.9 \pm 0.4$ & $0.7 \pm 0.1$ & $165 / 83$ \\
\hline IC 4329A & $1.88 \pm 0.02$ & $4.5 \pm 0.1$ & & & & $6.5 \pm 0.1$ & $0.5 \pm 0.3$ & $2.1 \pm 0.4$ & $0.39 \pm 0.05$ & $110 / 79$ \\
\hline NGC 3783 & $1.86 \pm 0.03$ & $2.5 \pm 0.1$ & & & & $6.4 \pm 0.7$ & $<0.4$ & $1.5 \pm 0.3$ & $0.41 \pm 0.08$ & $109 / 70$ \\
\hline NGC 5548 & $1.72 \pm 0.02$ & $1.51 \pm 0.04$ & & & & $6.2 \pm 0.1$ & $0.6 \pm 0.2$ & $1.1 \pm 0.2$ & $0.13 \pm 0.04$ & $69.3 / 58$ \\
\hline Mkn 509 & $1.75 \pm 0.02$ & $1.48 \pm 0.05$ & & & & $6.3 \pm 0.2$ & $<0.6$ & $0.7 \pm 0.2$ & $0.15 \pm 0.05$ & $70.9 / 62$ \\
\hline MR 2251-178 & $1.63 \pm 0.02$ & $0.97 \pm 0.08$ & & & & $6.3 \pm 0.3$ & $0.9 \pm 0.5$ & $0.9 \pm 0.3$ & $<0.01$ & $76.0 / 48$ \\
\hline NGC 3516 & $1.82 \pm 0.04$ & $1.7 \pm 0.2$ & & & $0.55^{2} \pm 0.10$ & $6.2 \pm 0.1$ & $<0.3$ & $0.9 \pm 0.2$ & $0.31 \pm 0.09$ & $58.2 / 60$ \\
\hline NGC 3227 & $1.79 \pm 0.04$ & $1.3 \pm 0.1$ & $2.7 \pm 0.9$ & & & $6.4 \pm 0.2$ & $0.6 \pm 0.5$ & $0.6 \pm 0.2$ & $0.41 \pm 0.09$ & $62.4 / 69$ \\
\hline NGC 4593 & $1.85 \pm 0.03$ & $1.25 \pm 0.05$ & & & & $6.4 \pm 0.1$ & $0.4 \pm 0.1$ & $1.0 \pm 0.1$ & $0.34 \pm 0.09$ & $77.8 / 59$ \\
\hline NGC 7469 & $1.88 \pm 0.04$ & $1.02 \pm 0.06$ & & & & $6.3 \pm 0.1$ & $<0.5$ & $0.5 \pm 0.1$ & $0.4 \pm 0.1$ & $65.4 / 53$ \\
\hline $3 \mathrm{C} 111$ & $1.67 \pm 0.01$ & $1.2_{-0.8}^{+0.2}$ & & & & $6.2 \pm 0.2$ & $0.7 \pm 0.2$ & $1.2 \pm 0.3$ & $<0.02$ & $102 / 72$ \\
\hline 3C 120 & $1.82 \pm 0.03$ & $1.50 \pm 0.01$ & & & & $6.5 \pm 0.2$ & $<0.7$ & $0.6 \pm 0.4$ & $0.24 \pm 0.06$ & $69.8 / 76$ \\
\hline $3 C 273$ & $1.69 \pm 0.01$ & $2.82 \pm 0.04$ & & & & $7.0_{-2.0}^{+0 *}$ & $0.9_{-0.6}^{+1.0}$ & $0.7 \pm 0.3$ & $0.07 \pm 0.03$ & $126 / 82$ \\
\hline Cen A & $1.83 \pm 0.01$ & $16.0 \pm 0.3$ & $16.9 \pm 0.3$ & & & $6.38 \pm 0.09$ & $<0.5$ & $4.9 \pm 0.7$ & $<0.005$ & $143 / 83$ \\
\hline NGC 5506 & $1.93 \pm 0.03$ & $3.6 \pm 0.2$ & $1.9 \pm 0.5$ & & & $6.1 \pm 0.2$ & $1.0 \pm 0.2$ & $5.4 \pm 0.9$ & $1.2 \pm 0.1$ & $118 / 69$ \\
\hline MCG-5-23-16 & $1.84 \pm 0.03$ & $3.3 \pm 0.2$ & $3.5 \pm 0.5$ & & & $6.4 \pm 0.6$ & $0.2 \pm 0.1$ & $1.8 \pm 0.3$ & $0.4 \pm 0.1$ & $81.5 / 67$ \\
\hline NGC 4507 & $1.72 \pm 0.04$ & $1.8 \pm 0.3$ & $87 \pm 2$ & & & $6.45 \pm 0.05$ & $<0.4$ & $1.4 \pm 0.2$ & $0.4 \pm 0.1$ & $126 / 70$ \\
\hline NGC 7582-REFL & $1.79 \pm 0.10$ & $0.34 \pm 0.06$ & $14 \pm 3$ & & & $6.2 \pm 0.1$ & $<0.5$ & $0.7 \pm 0.2$ & $3.3_{-0.9}^{+1.6}$ & $36.3 / 33$ \\
\hline & & & & $\Gamma_{\mathrm{SXPL}^{3}}$ & $A_{\mathrm{SXPL}}{ }^{1}\left(10^{-2}\right)$ & & & & & \\
\hline Circinus & $2.01 \pm 0.06$ & $10 \pm 2$ & $690 \pm 50$ & $2.01 \pm 0.06$ & $0.44 \pm 0.02$ & $6.44 \pm 0.01$ & $<0.15$ & $4.7 \pm 0.2$ & $0.53 \pm 0.04$ & $138 / 62$ \\
\hline NGC 4945 & $1.71 \pm 0.03$ & $3.0 \pm 0.4$ & $670 \pm 35$ & $4.0 \pm 0.5$ & $2_{-1}^{+2}$ & $6.4 \pm 0.1$ & $0.4 \pm 0.2$ & $0.7 \pm 0.2$ & $0.24 \pm 0.05$ & $175 / 81$ \\
\hline
\end{tabular}

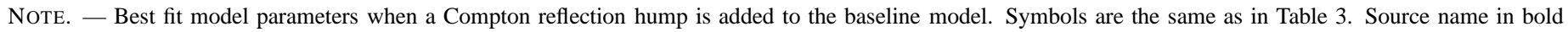
indicates that we conclude this model is the best description of the spectrum. An asterisk (*) indicates parameter pegged at hard limit.

${ }^{1}$ Power law normalization ( $\mathrm{ph} \mathrm{keV}^{-1} \mathrm{~cm}^{-2} \mathrm{~s}^{-1}$ at $1 \mathrm{keV}$ )

${ }^{2}$ Partial covering warm absorber with $\log (\xi)$ fixed at 2.19 and column density fixed at $2 \times 10^{23} \mathrm{~cm}^{-2}$ but covering fraction left as a free parameter.

${ }^{3}$ SXPL parameters are for the soft X-ray power law due to leaked or scattered emission.

TABLE 5

ROLLOVER MODELS

\begin{tabular}{|c|c|c|c|c|c|c|c|c|c|c|c|}
\hline Source & $\bar{\Gamma}$ & $\begin{array}{c}A^{1} \\
\left(10^{-2}\right)\end{array}$ & $\begin{array}{c}N_{\mathrm{H}} \\
\left(10^{22} \mathrm{~cm}^{-2}\right)\end{array}$ & $\Gamma_{\mathrm{SXPL}}{ }^{2}$ & $\begin{array}{l}A_{\mathrm{SXPL}}{ }^{2} \\
\left(10^{-2}\right)\end{array}$ & $\begin{array}{c}\text { Fe Line } \\
\text { Energy }(\mathrm{keV})\end{array}$ & $\begin{array}{c}\sigma \\
(\mathrm{keV})\end{array}$ & $\begin{array}{c}I_{\mathrm{Fe}}\left(10^{-4}\right) \\
\left(\mathrm{ph} \mathrm{cm}^{-2} \mathrm{~s}^{-1}\right)\end{array}$ & $R$ & $\begin{array}{l}E_{\text {roll }}^{3} \\
(\mathrm{keV})\end{array}$ & $\chi^{2} / \mathrm{dof}$ \\
\hline MR 2251-178 & $1.56 \pm 0.03$ & $0.91_{-0.07}^{+0.03}$ & & & & $6.2_{-0.6}^{+0.3}$ & $0.8 \pm 0.6$ & $0.8 \pm 0.5$ & $<0.06$ & $100_{-30}^{+40}$ & $65.8 / 47$ \\
\hline Circinus & $1.2 \pm 0.2$ & $1.2_{-0.6}^{+0.9}$ & $920_{-150}^{+120}$ & $2.5 \pm 0.4$ & $1.1 \pm 0.4$ & $6.42 \pm 0.01$ & $0.20 \pm 0.04$ & $5.2 \pm 0.1$ & $1.1 \pm 0.3$ & $41_{-10}^{+6}$ & $47.1 / 62$ \\
\hline
\end{tabular}

Note. - Symbols are the same as in Table 3. The Fe line parameters for these sources do not vary greatly when a rollover is added. See Tables 3 and 4 for these values. Source name in bold indicates that we conclude this model is the best description of the spectrum.

${ }^{1}$ Power law normalization ( $\mathrm{ph} \mathrm{keV}^{-1} \mathrm{~cm}^{-2} \mathrm{~s}^{-1}$ at $\left.1 \mathrm{keV}\right)$

${ }^{2}$ SXPL parameters are for the soft X-ray power law due to leaked or scattered emission.

${ }^{3} E_{\text {roll }}$ is the energy of the high-energy rollover.

The inclination was set to $30^{\circ}$ for Seyfert $1-1.5$ 's and $45^{\circ}$ for Seyfert 2 's. Results are listed in Table 4 The value $R$ given by the model is the fraction of light reflected assuming an isotropic X-ray source above a semi-infinite slab such as a disk covering $50 \%$ of the sky as viewed by the illuminating source. Significant improvement in fit was found for all objects except MR 2251-178, 3C 111, and Cen A.

Next we tested for the presence of high-energy continuum cutoffs. We tested two forms for the cut-off, "CUTOFFPL" and "HIGHECUT". CUTOFFPL (which is a bit of a misnomer) has a slow rollover where the continuum at $E_{\text {roll }}$ is $1 / e$ times the initial value. HIGHECUT has a much more abrupt cut-off governed by the following equations: $A(E)$ $=e^{\left(E_{\mathrm{cut}}-E\right) / E_{\text {fold }}}$ for $E>E_{\text {cut }} ; A(E)=1$ for $E<E_{\text {cut }}$. Since we did not see evidence for such abrupt cut-offs in the data, results found using this model are not presented in this paper. $\mathrm{E}_{\text {roll }}$ was limited to a hard lower bound of $50 \mathrm{keV}$ in all cases with the exception of Circinus which shows evidence for a rollover at slightly lower energies $(\sim 40$ $\mathrm{keV}$ ). We included the PEXRAV component in these fits with $\Gamma$ and $E_{\text {roll }}$ tied to those of CUTOFFPL.

Best-fit CUTOFFPL results are listed in Table 5 We found sig- nificant improvement in fit $\left(\Delta \chi^{2}>50\right)$ for two objects, Circinus and NGC 4945 and marginal evidence $\left(\Delta \chi^{2} \sim 10\right)$ for a rollover in MR 2251-178 (see notes on individual sources). In all other cases, we found only lower limits for rollovers as $\chi^{2}$ did not improve, nor was there improvement in data/model residuals at high energies. Unfortunately, for the two cases (both Compton-thick Seyfert 2's) where the rollover substantially improved the fit, systematic degeneracies present between $\Gamma, E_{\text {roll }}$ and $R$ lead to unrealistically low values of the photon index when a rollover is modeled. For this reason we present analysis on both models.

We include contour plots of $N_{\mathrm{H}}$ versus $\Gamma, R$ versus $\Gamma$ and $E_{\text {roll }}$ versus $\Gamma$ (in instances where a rollover was detected) in Figure 4 for Seyfert 2's to identify potential model degeneracies. In nearly all cases statistical degeneracies are minimal, the one exception being NGC 7582, for which the reflection strength is very poorly constrained. Additionally, Figure 5 shows $R$ versus $\Gamma$ for all objects with well-constrained/realistic values, ie. excluding the Comptonthick sources NGC 7582, NGC 4945 and Circinus.

Spectra are shown in Figures 628 along with the best-descriptor 
TABLE 6

BLAZAR BEST-FIT PARAMETERS

\begin{tabular}{lccccc}
\hline \hline Source & $\Gamma_{1}$ & $\begin{array}{c}\text { Break } \\
\text { Energy } \\
(\mathrm{keV})\end{array}$ & $\Gamma_{2}$ & $\mathrm{~A}^{1}$ & $\chi^{2} /$ dof \\
& & & & \\
\hline 3C 454.3 & $1.67 \pm 0.05$ & & & $1.8 \pm 0.2^{2}$ & $18.2 / 38$ \\
Mkn 421 & $2.49 \pm 0.08$ & $7.1 \pm 0.8$ & $2.79 \pm 0.01$ & $31 \pm 4$ & $94.6 / 79$ \\
1ES 1959+650 & $2.01 \pm 0.02$ & $5.7 \pm 0.8$ & $2.12 \pm 0.01$ & $6.2 \pm 0.3^{3}$ & $40.7 / 62$
\end{tabular}

Note. - Best fit model parameters for the jet-dominated sources in our sample. Power law normalization refers to the normalization at $1 \mathrm{keV}$ for the simple power law fit and the normalization at the break energy for the broken power law fits. For best fit parameters of 3C 273 please see Table 4

${ }^{1}$ Power law normalization $\left(10^{-2} \mathrm{ph} \mathrm{keV}^{-1} \mathrm{~cm}^{-2} \mathrm{~s}^{-1}\right)$ at $1 \mathrm{keV}$ for a power law and at the break energy for a broken power law.

${ }^{2}$ Off-source pointing taken into account, see Section 2.2 for details.

${ }^{3}$ Uncertainty determined with the break energy held fixed.

model in panel (a), $\chi$ values for the base model in panel (b), and any models that show improvement past the base in subsequent panels. Data to model ratios for the best-fit reflection and rollover models that demonstrate the extra curvature in Circinus and NGC 4945 that indicates the presence of a high-energy rollover are shown in Figures 29 and 30.

\subsection{Blazars}

Blazars are jet-dominated sources whose continua are very slowly bending through the X-ray bandpass, and whose X-ray spectra are typically fit by power laws, broken power laws, or other slowly bending continuum models. Fit results are listed in Table 6 for all but 3C 273 which we tested for evidence of an Fe line and Compton hump based on previous detections of these parameters (see Appendix A.20 for details). Best fit parameters for 3C 273 are listed in Table 4

For $3 \mathrm{C} 454.3$, a power law absorbed only by $N_{\mathrm{H}}$, Gal yielded a good fit. For Mkn 421 and 1ES 1959+650, we found that a broken power law fit, absorbed only by $N_{\mathrm{H} \text {, Gal }}$, yielded a significantly better fit compared to an unbroken power law.

Broadband SED fitting to constrain synchrotron/inverse Compton emission parameters is beyond the scope of this paper. Thus we do not discuss this class of objects further, however it is our hope that future work may be done incorporating time-averaged spectral properties in other bands.

\subsection{Further Analysis}

Additional information for the "best descriptor" models is given in Tables 7 and 8 which list Fe line equivalent widths (EW's) and flux/luminosity information respectively. Luminosities were calculated following, e.g., Alexander et al. (2003, their Equation (1)). Luminosity distances were taken from the NASA Extragalactic Database. For each object, the mean of the available redshiftindependent estimates was used if available, otherwise the luminosity distance based on redshift (using the $3 \mathrm{~K}$ cosmic microwave background radiation as a reference frame) was used.

\section{DISCUSSION}

Compared to X-ray data below $\sim 10 \mathrm{keV}$, higher energies have been relatively unexplored because of the difficulty in obtaining simultaneous high-quality spectra above $20 \mathrm{keV}$. By using RXTE PCA + HEXTE monitoring data for X-ray bright sources, we have been able to construct spectra featuring broadband sensitivity that extends up to $\geq 100 \mathrm{keV}$, and long total exposure times.

HEXTE offers several advantages compared to other instruments which operated or are operating above $20 \mathrm{keV}$. HEXTE has realtime background measurement, as opposed to relying on modeling as with the Suzaku HXD. BeppoSAX PSD had a collecting area equivalent to only one HEXTE cluster but with significantly less background due to its equatorial orbit, while OSSE, INTEGRAL-IBIS, and Swift-BAT have much higher background due to larger fields of view.

Because the RXTE monitoring has spanned a very long baseline (more than a decade for several sources), the time-averaged spectral
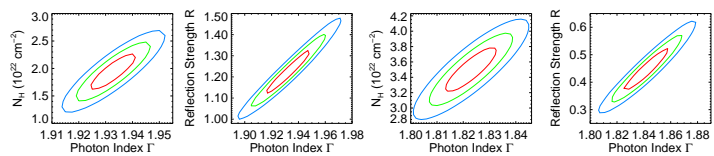

NGC 5506

MCG-5-23-16
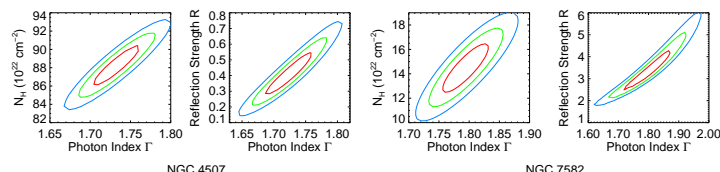

NGC 4507
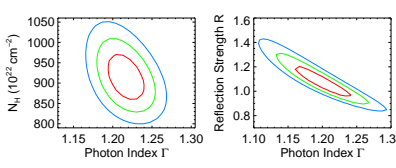

NGC 758
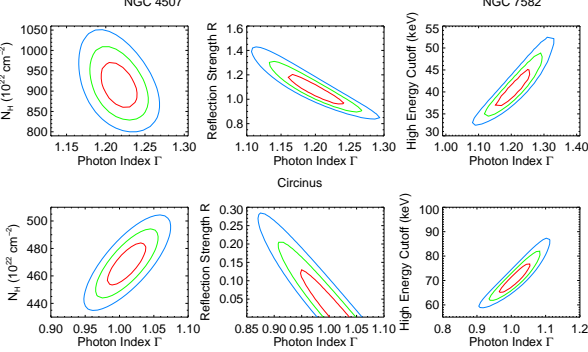

NGC 4945

FIG. 4.- Contour plots of various parameters for Seyfert 2's, illustrating the degree of statistical degeneracy seen in these objects.

properties derived can be taken to be true averages over time and over a wide range of fluxes. Judging by the $2-10 \mathrm{keV}$ light curves, for most sources, we have sampled the full range typically displayed by a given source in the recent past. Swift-BAT, with its large field of view, has also been able to provide long-term monitoring in the 14-195 $\mathrm{keV}$ energy range since 2005 , however the spectra currently have only eight channels which adds to the difficulty of fitting multi-component spectra.

One of our main goals was to obtain constraints on the strength of the Compton reflection component, which we detected in the majority of Seyferts (16-17 out of 20 Seyferts, depending on the form of the model used). As detailed below, we found an average reflection strength $\langle R\rangle$ of $0.35 \pm 0.16$ for Seyfert 1's and $0.67 \pm 0.46$ for Compton-thin Seyfert 2's. The implications for the geometry of the Compton-thick gas are discussed below in Section 4.2. Another goal was to search for high-energy rollovers in the power-law continuum, expected if the power-law is produced by thermal Comptonization in an X-ray corona. We found evidence for high-energy rollovers in only three sources in the sample, and we caution that the evidence is somewhat tentative in all three cases. Lower limits to $E_{\text {roll }}$ were obtained for the remaining Seyferts and the implications for the X-ray corona are discussed in Section 4.3.

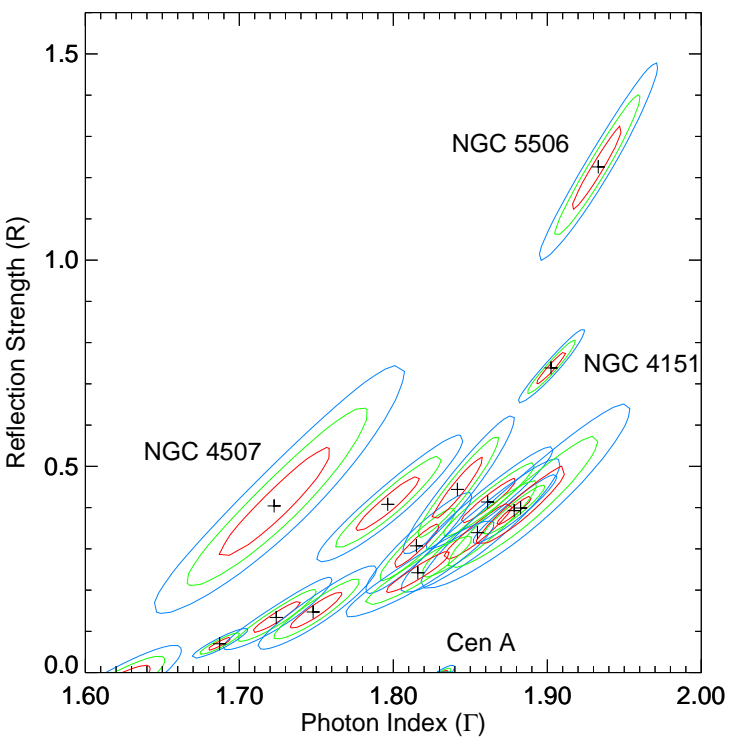

FIG. 5.- Contour plot of $R$ versus $\Gamma$ for all objects with well-constrained values, ie. excluding Compton-thick sources. The scatter in this plot seems to indicate that there is no strong correlation between $R$ and $\Gamma$ and that our fits are robust in determining these parameters. 


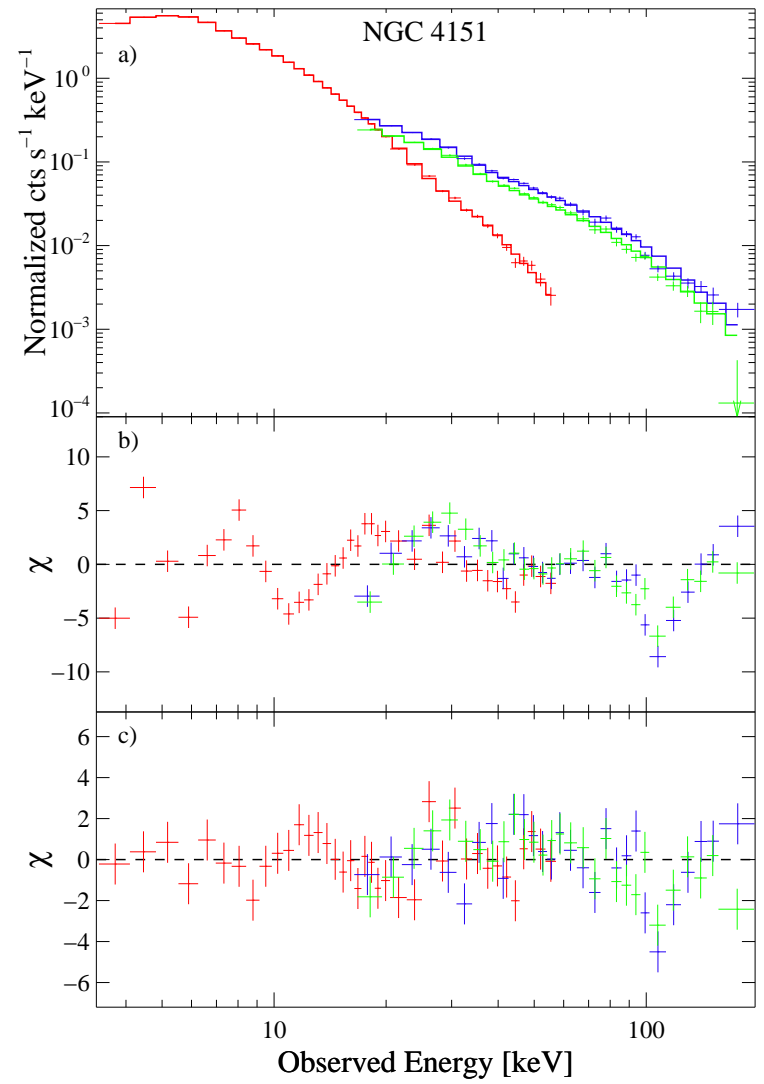

FIG. 6.- Data, model and data-model residuals for NGC 4151. Panel (a) shows the PCA and HEXTE data along with the best-fit model (solid line); panel (b) shows residuals for the baseline model; and panel (c) shows residuals for the best-fit model (parameters for the best-fit model are listed in Table 4).

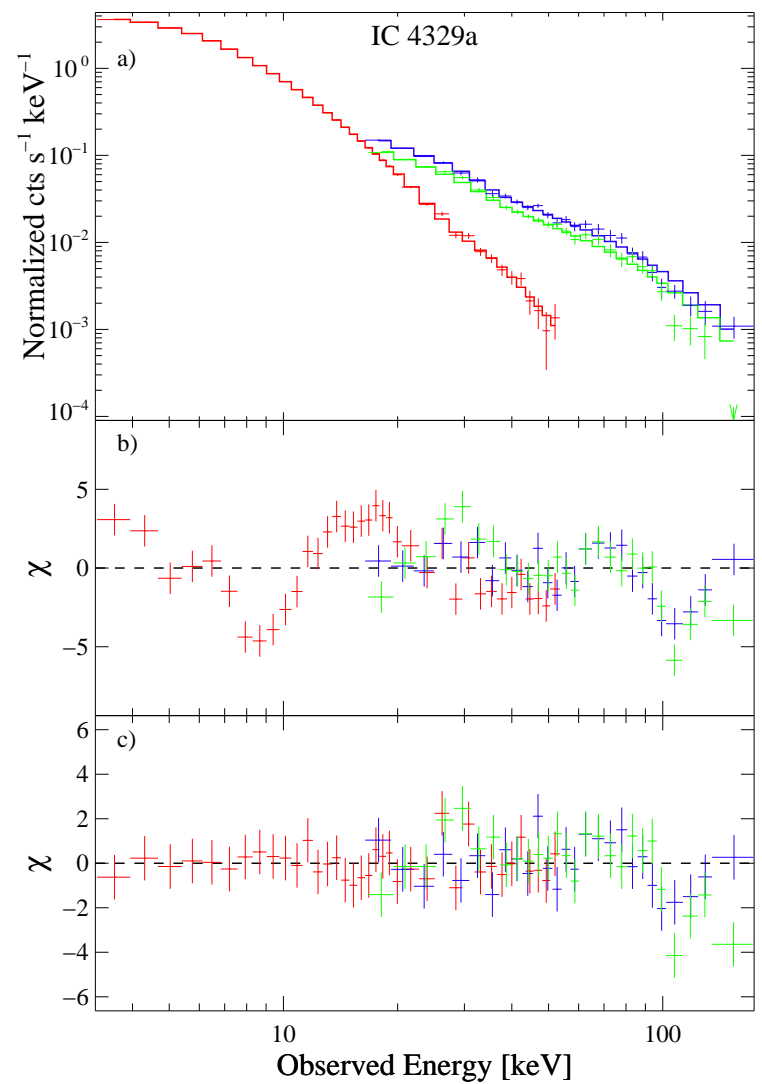

FIG. 7.- Data, model and data-model residuals for IC 4329a. Panel (a) shows the PCA and HEXTE data along with the best-fit model (solid line); panel (b) shows residuals for the baseline model; and panel (c) shows residuals for the best-fit model (parameters for the best-fit model are listed in Table 4).

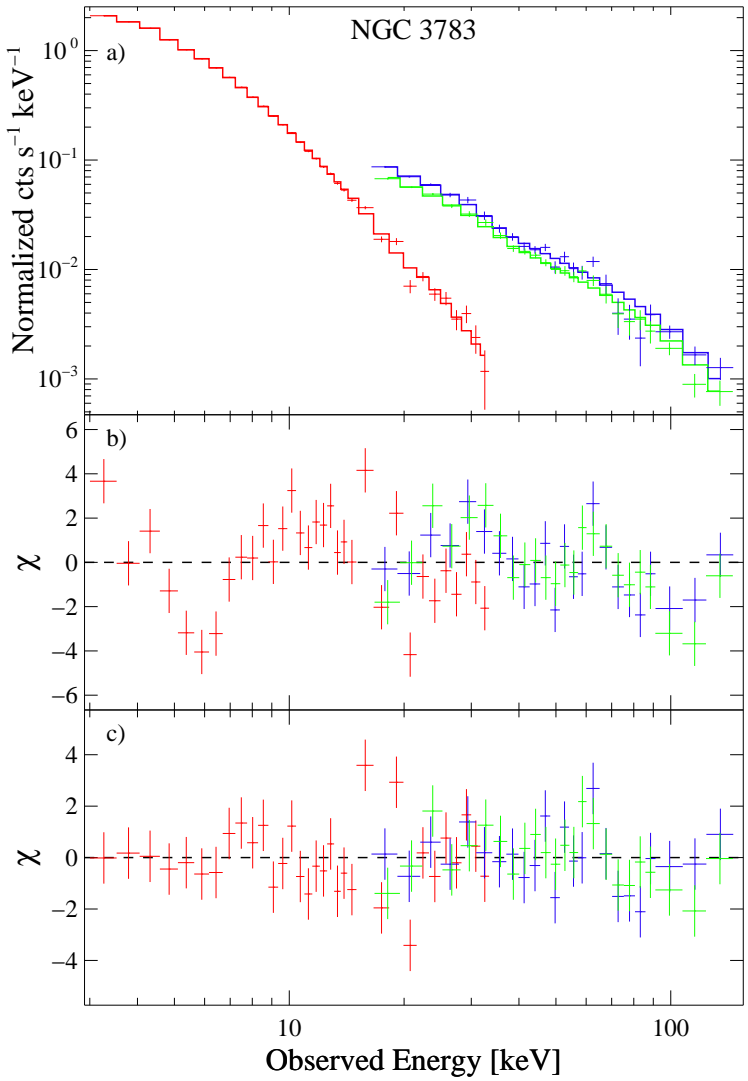

FIG. 8.- Data, model and data-model residuals for NGC 3783. Panel (a) shows the PCA and HEXTE data along with the best-fit model (solid line); panel (b) shows residuals for the baseline model; and panel (c) shows residuals for the best-fit model (parameters for the best-fit model are listed in Table 4).

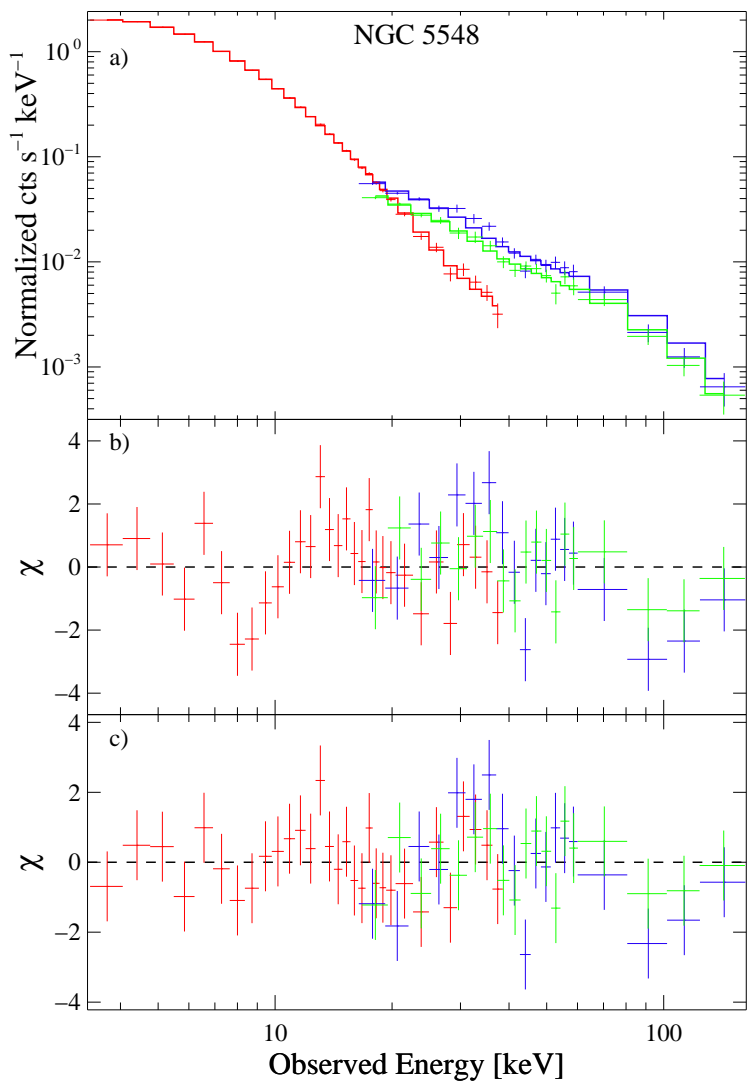

FIG. 9.- Data, model and data-model residuals for NGC 5548. Panel (a) shows the PCA and HEXTE data along with the best-fit model (solid line); panel (b) shows residuals for the baseline model; and panel (c) shows residuals for the best-fit model (parameters for the best-fit model are listed in Table 4 ). 




FIG. 10.- Data, model and data-model residuals for Mkn 509. Panel (a) shows the PCA and HEXTE data along with the best-fit model (solid line); panel (b) shows residuals for the baseline model; and panel (c) shows residuals for the best-fit model (parameters for the best-fit model are listed in Table 4).

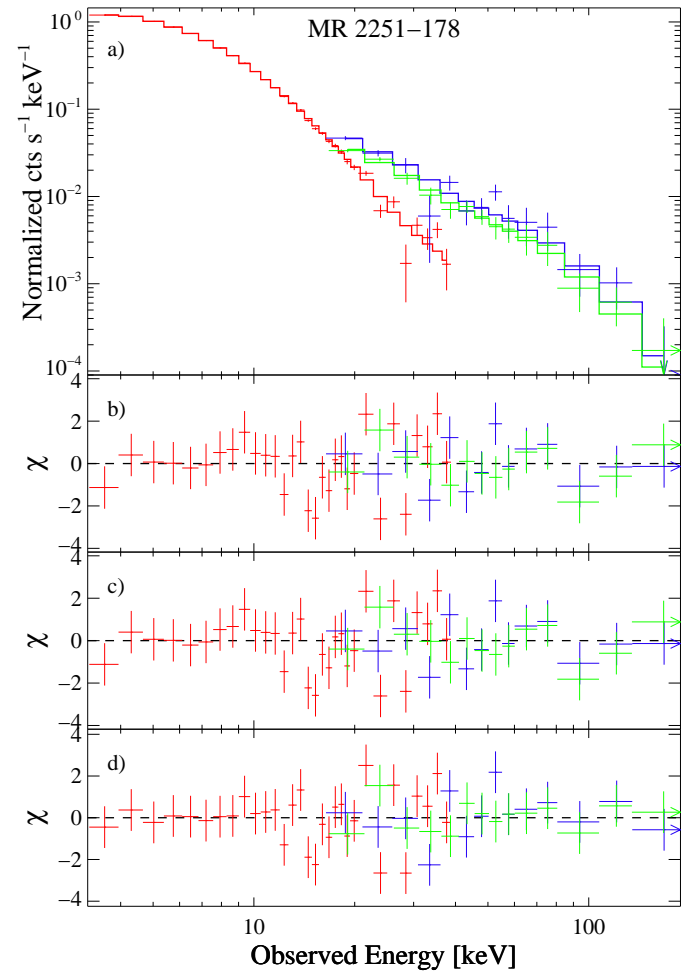

FIG. 11.- Data, model and data-model residuals for MR2251-178. Panel (a) shows the PCA and HEXTE data along with the best-fit model (solid line); panel (b) shows residuals for the baseline model; panel (c) showss residuals for the PEXRAV model; and panel d) shows residuals for the best-fit model with PEXRAV and a high-energy rollover (parameters for the best-fit model are listed in Table 5 .

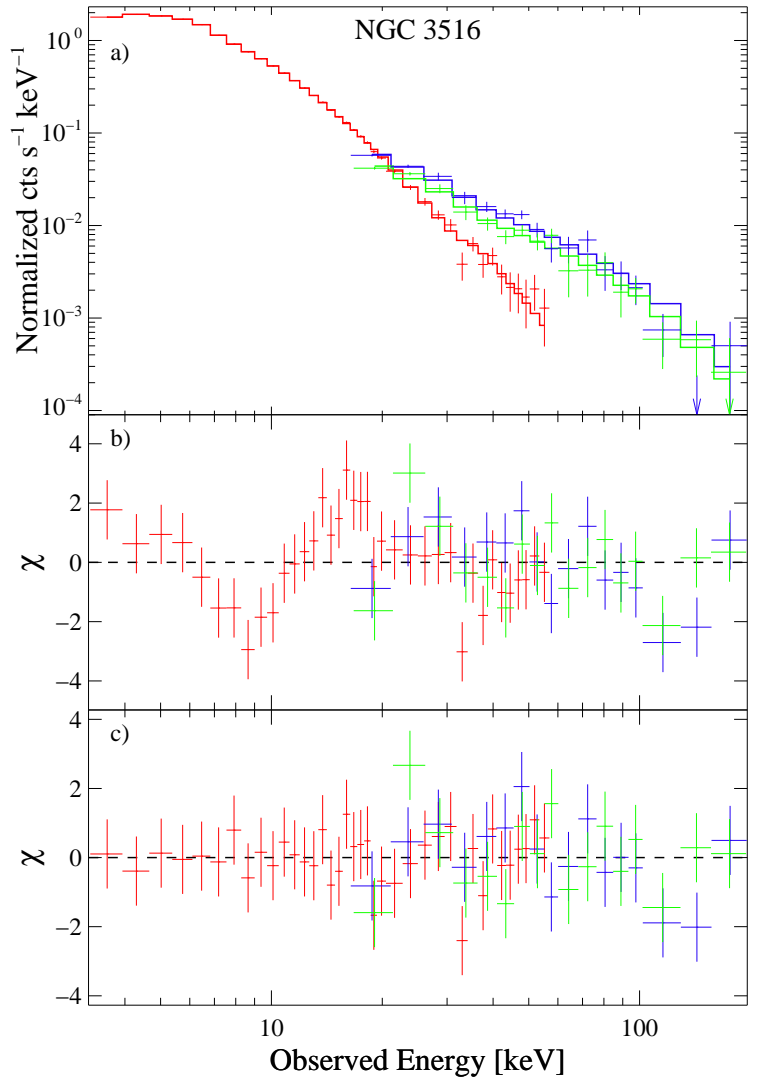

FIG. 12.- Data, model and data-model residuals for NGC 3516. Panel (a) shows the PCA and HEXTE data along with the best-fit model (solid line); panel (b) shows residuals for the baseline model; and panel (c) shows residuals for the best-fit model (parameters for the best-fit model are listed in Table 4 ).

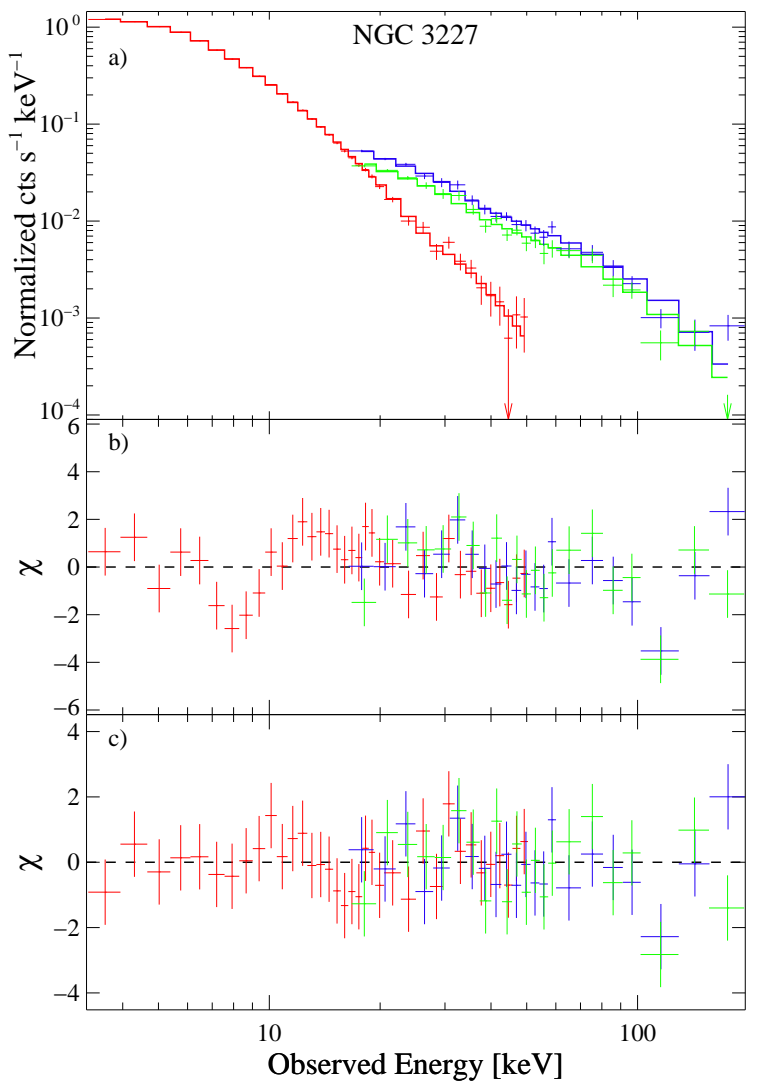

FIG. 13.- Data, model and data-model residuals for NGC 3227. Panel (a) shows the PCA and HEXTE data along with the best-fit model (solid line); panel (b) shows residuals for the baseline model; and panel (c) shows residuals for the best-fit model (parameters for the best-fit model are listed in Table 4 . 


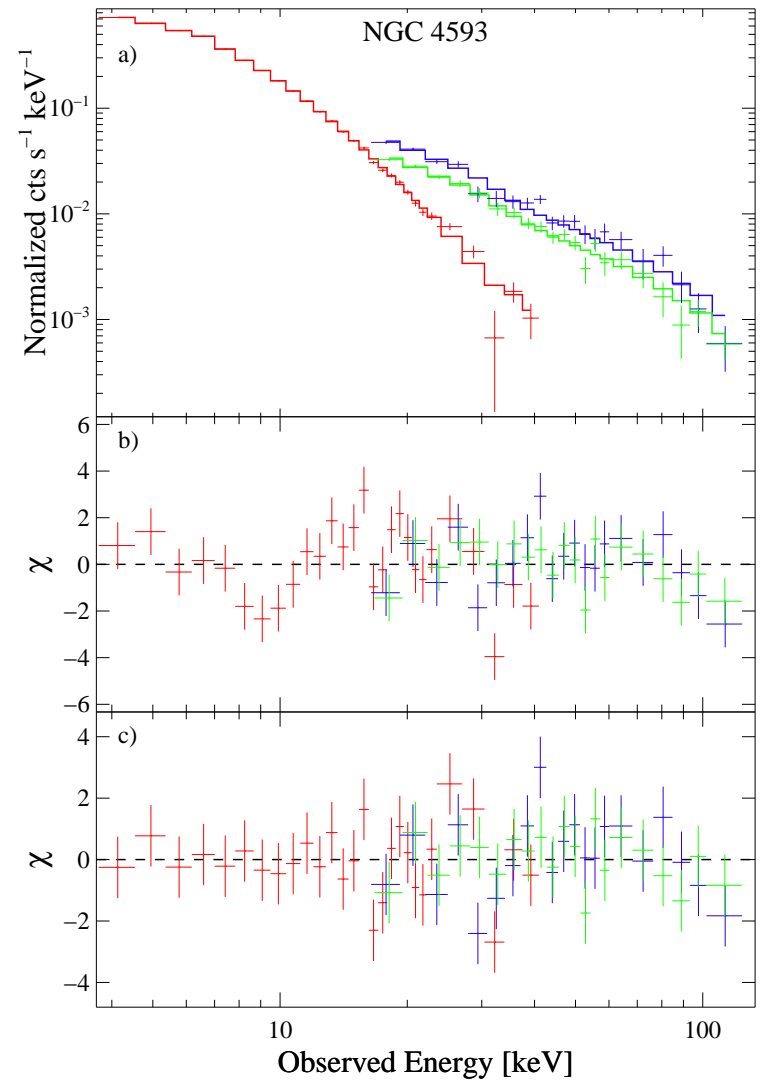

FIG. 14.- Data, model and data-model residuals for NGC 4593. Panel (a) shows the PCA and HEXTE data along with the best-fit model (solid line); panel (b) shows residuals for the baseline model; and panel (c) shows residuals for the best-fit model (parameters for the best-fit model are listed in Table 4).

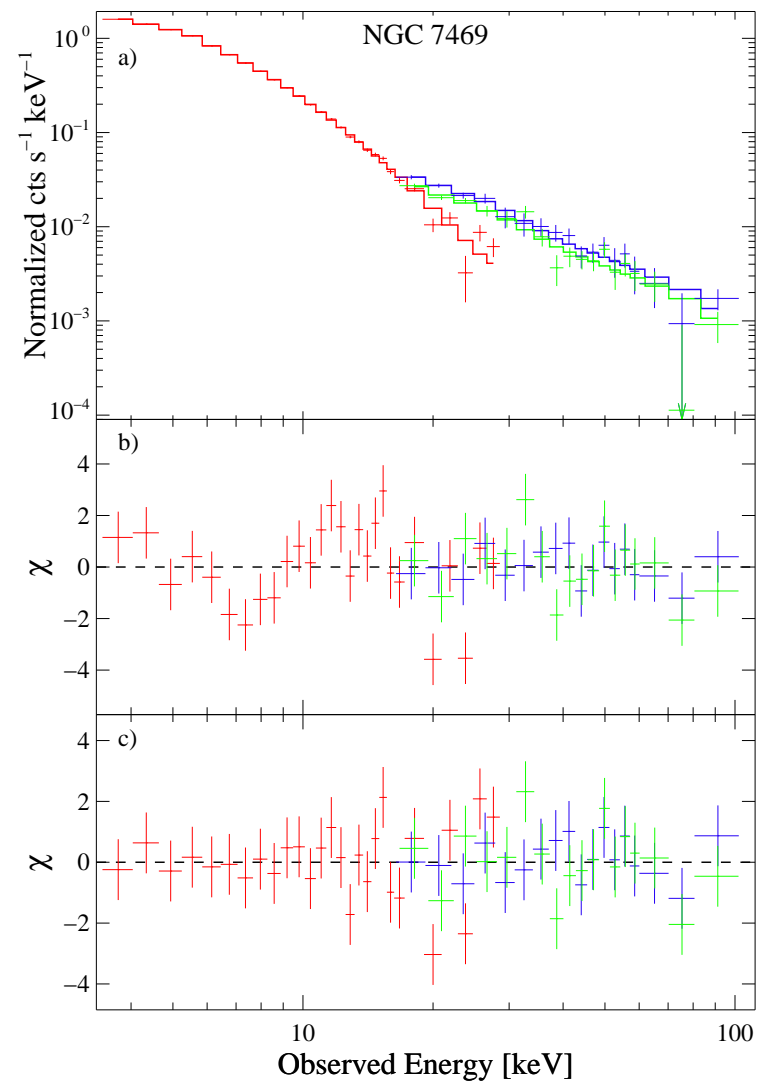

FIG. 15.- Data, model and data-model residuals for NGC 7469. Panel (a) shows the PCA and HEXTE data along with the best-fit model (solid line); panel (b) shows residuals for the baseline model; and panel (c) shows residuals for the best-fit model (parameters for the best-fit model are listed in Table 4 .

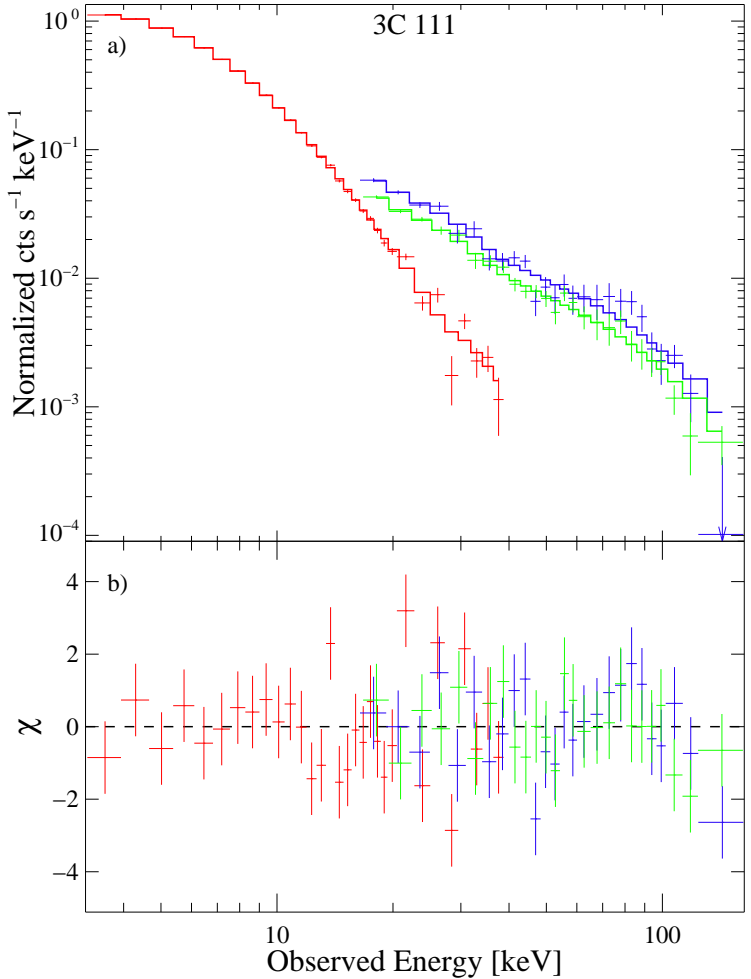

FIG. 16.- Data, model and data-model residuals for 3C 111. Panel (a) shows the PCA and HEXTE data along with the best-fit model (solid line); panel (b) shows residuals for the baseline model which is also the best-fit model for this source (parameters are listed in Table 3 .

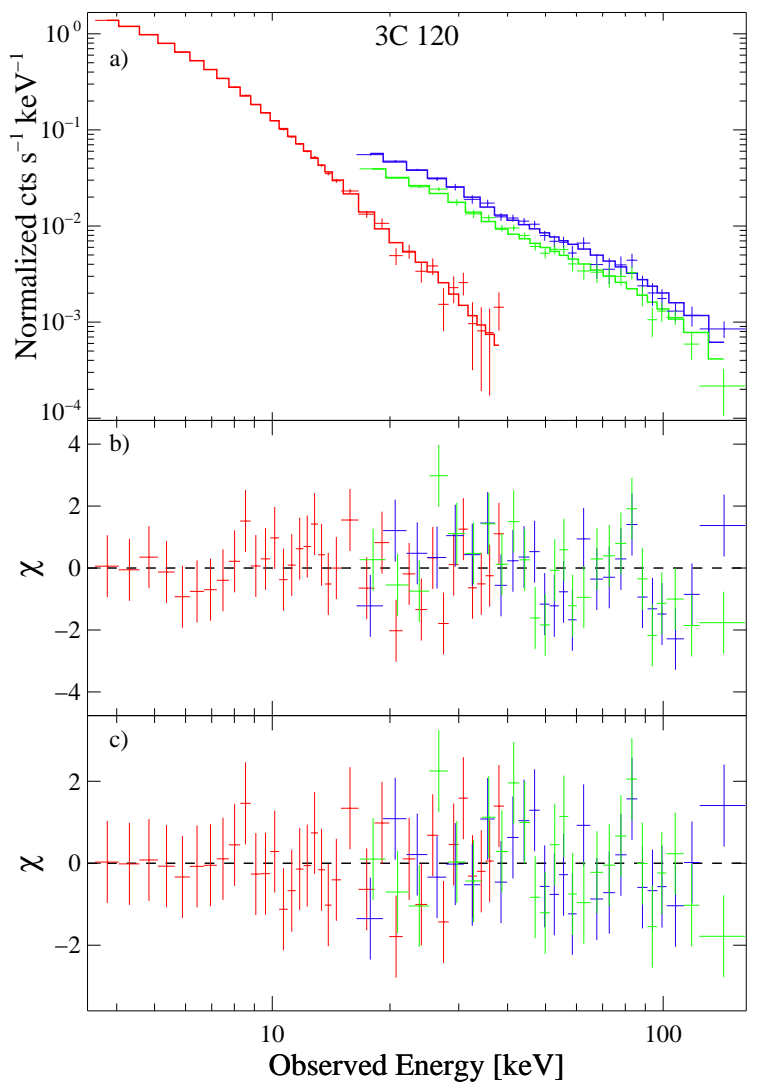

FIG. 17.- Data, model and data-model residuals for 3C 120. Panel (a) shows the PCA and HEXTE data along with the best-fit model (solid line); panel (b) shows residuals for the baseline model; and panel (c) shows residuals for the best-fit model (parameters for the best-fit model are listed in Table 4 ). 


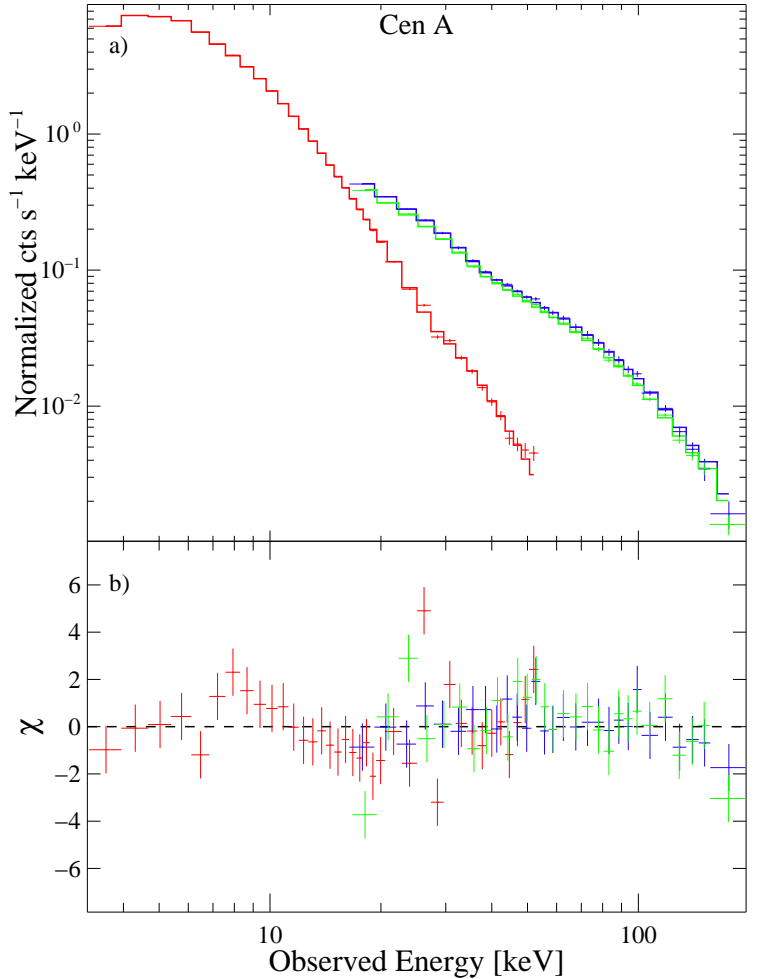

FIG. 18. - Data, model and data-model residuals for Cen A. Panel (a) shows the PCA and HEXTE data along with the best-fit model (solid line); panel (b) shows residuals for the baseline model which is also the best-fit model for this source (parameters are listed in Table 3 .

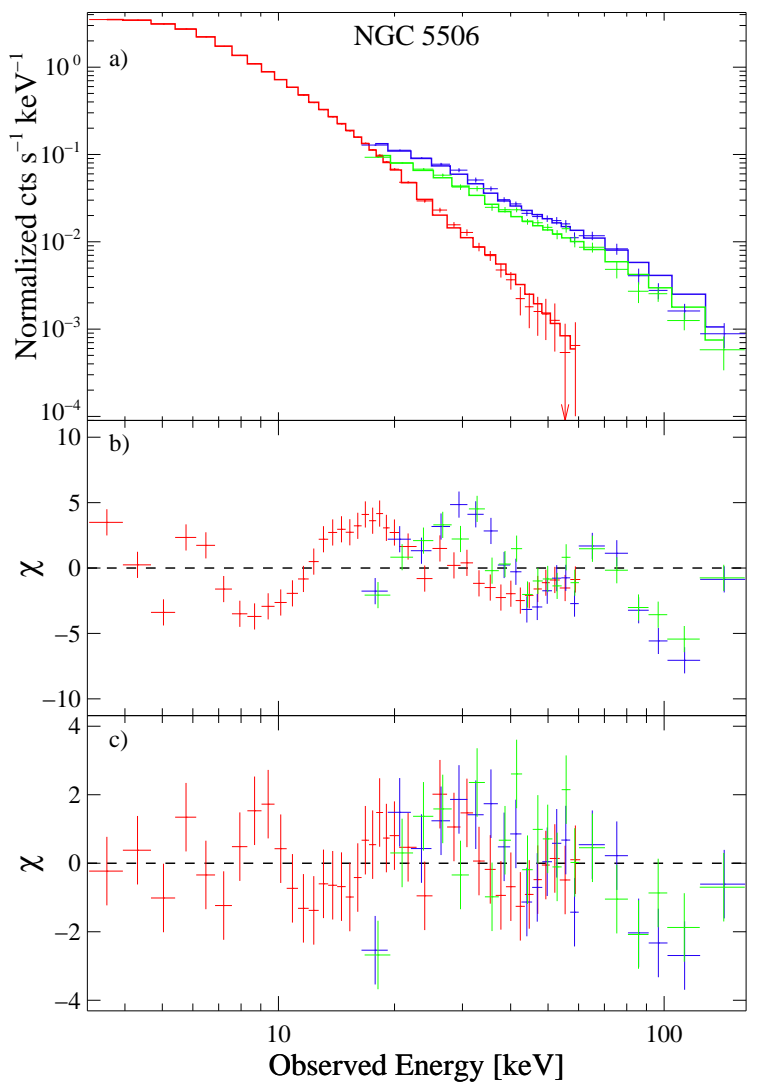

FIG. 19.- Data, model and data-model residuals for NGC 5506. Panel (a) shows the PCA and HEXTE data along with the best-fit model (solid line); panel (b) shows residuals for the baseline model; and panel (c) shows residuals for the best-fit model (parameters for the best-fit model are listed in Table 4 ).

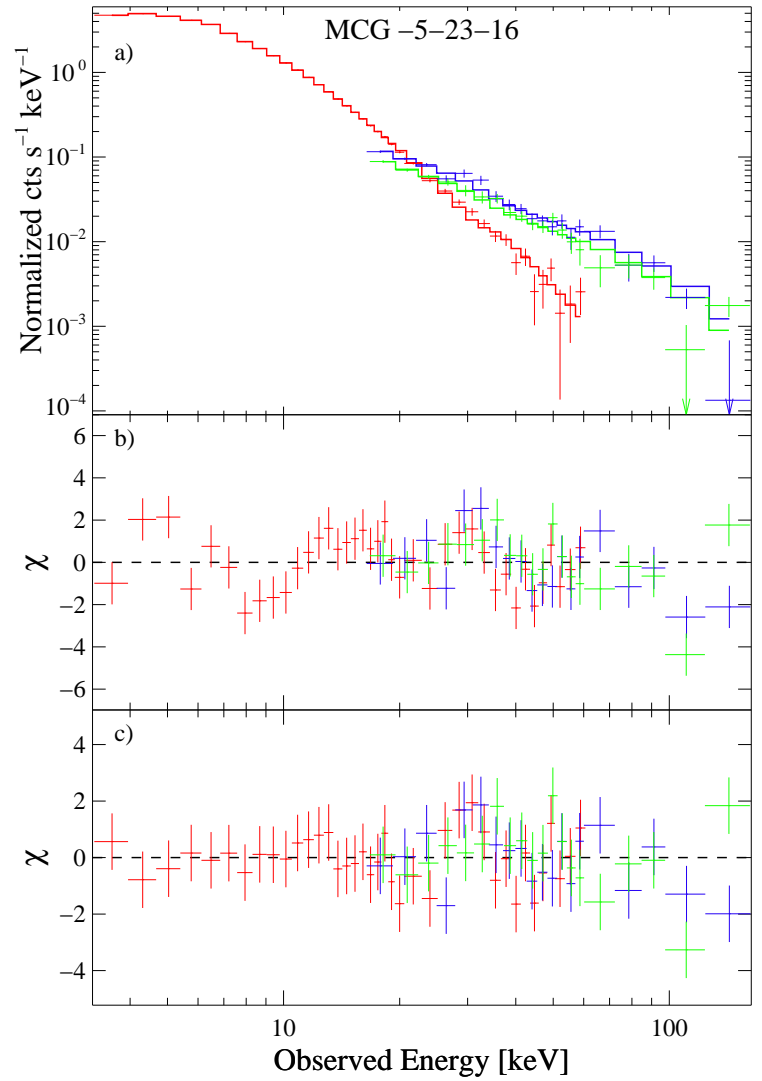

FIG. 20.- Data, model and data-model residuals for MCG-5-23-16. Panel (a) shows the PCA and HEXTE data along with the best-fit model (solid line); panel (b) shows residuals for the baseline model; and panel (c) shows residuals for the best-fit model (parameters for the best-fit model are listed in Table 4).

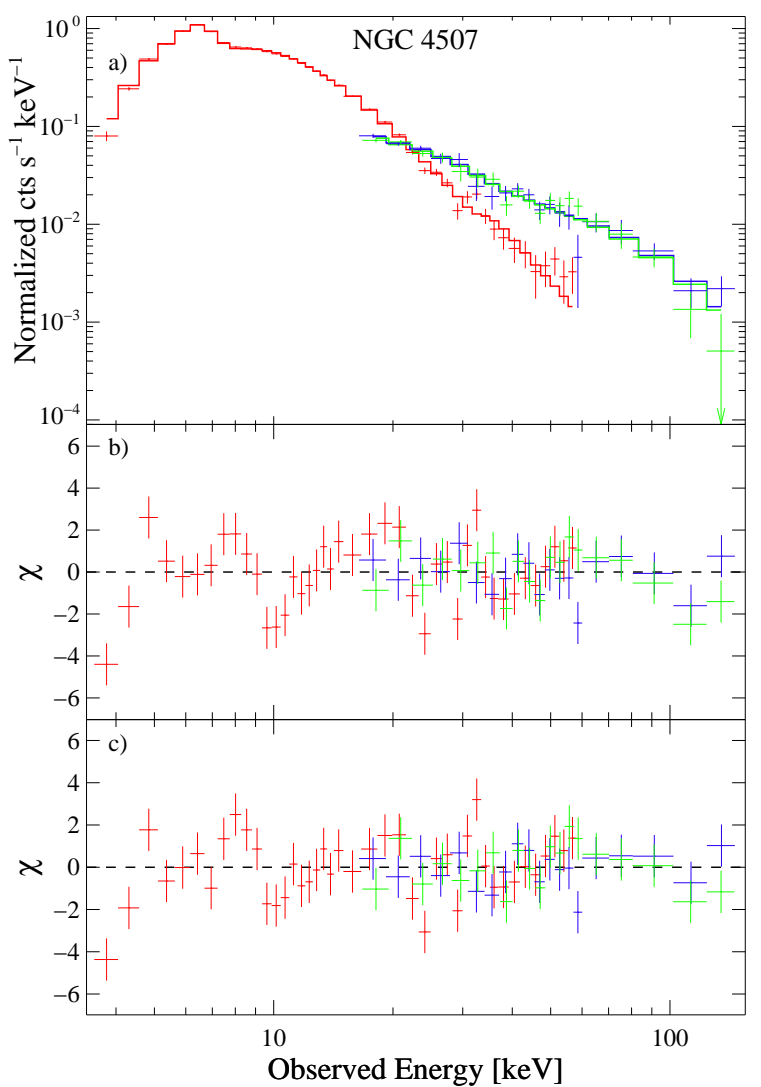

FIG. 21.- Data, model and data-model residuals for NGC 4507. Panel (a) shows the PCA and HEXTE data along with the best-fit model (solid line); panel (b) shows residuals for the baseline model; and panel (c) shows residuals for the best-fit model (parameters for the best-fit model are listed in Table 4). 


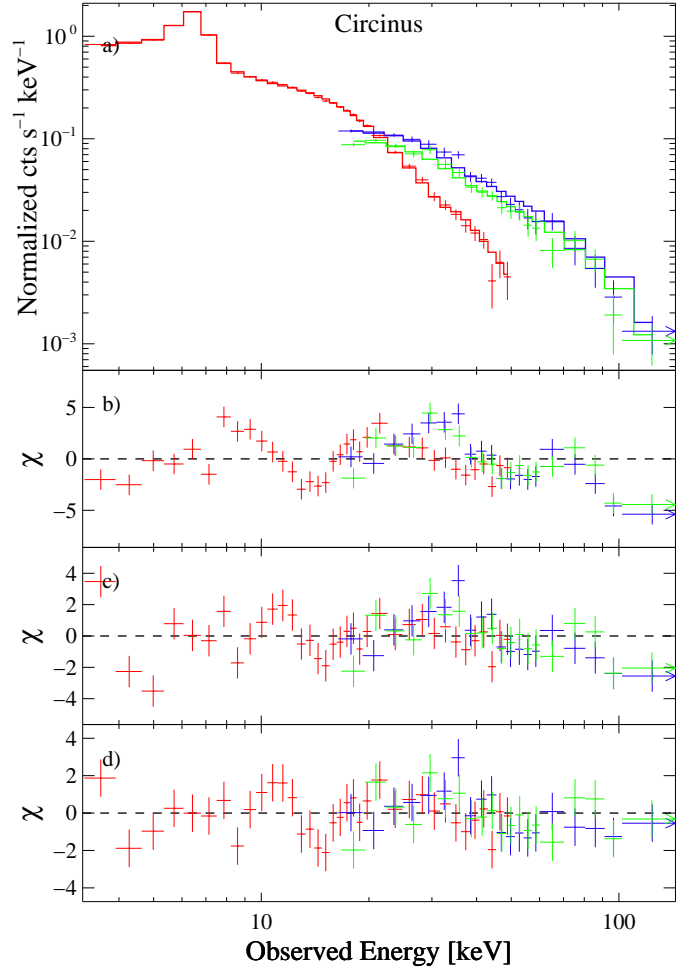

FIG. 22.- Data, model and data-model residuals for Circinus. Panel (a) shows the PCA and HEXTE data along with the best-fit model (solid line); panel (b) shows residuals for the baseline model; panel (c) showss residuals for the PEXRAV model; and panel d) shows residuals for the best-fit model with PEXRAV and a high-energy rollover (parameters for the best-fit model are listed in Table 5 .

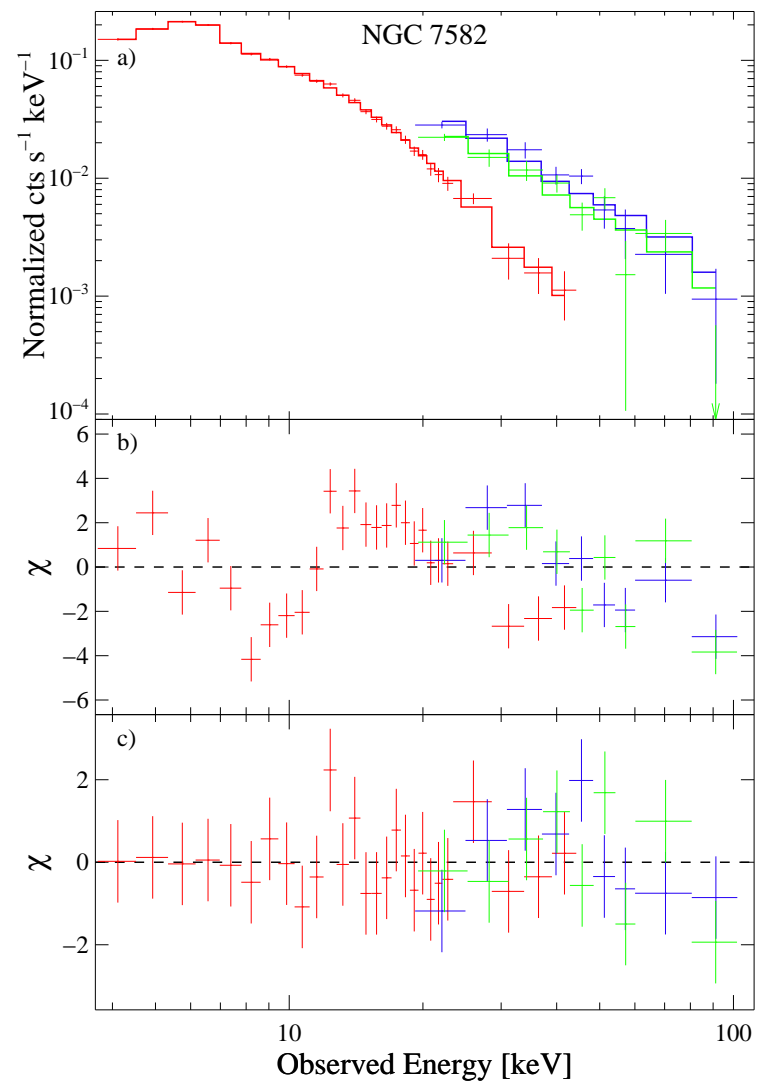

FIG. 23.- Data, model and data-model residuals for NGC 7582. Panel (a) shows the PCA and HEXTE data along with the best-fit model (solid line); panel (b) shows residuals for the baseline model; and panel (c) shows residuals for the best-fit model (parameters for the best-fit model are listed in Table 4).

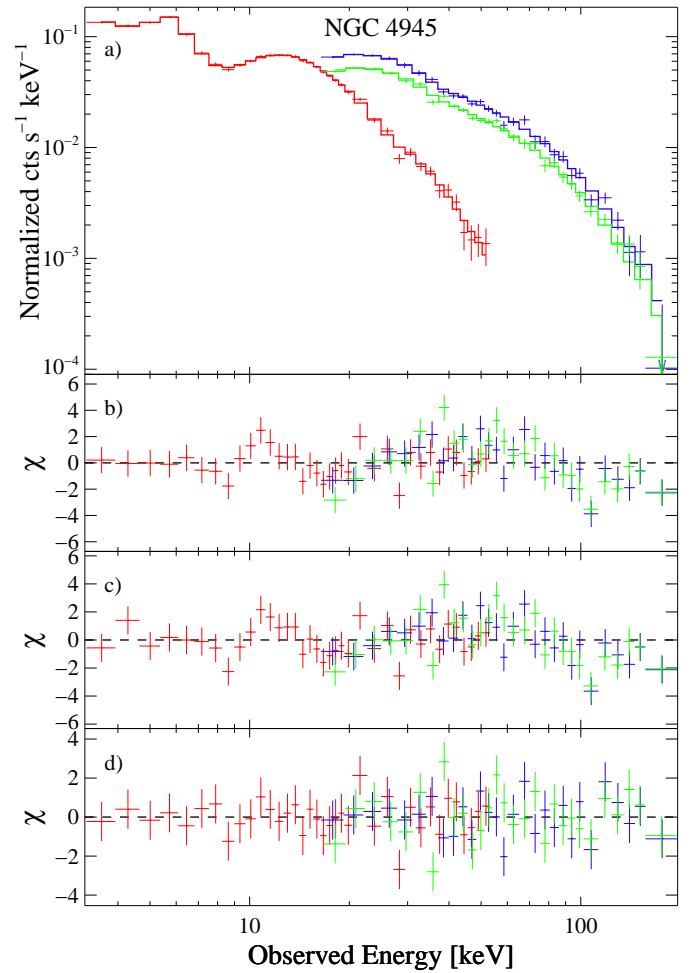

FIG. 24.- Data, model and data-model residuals for NGC 4945. Panel (a) shows the PCA and HEXTE data along with the best-fit model (solid line); panel (b) shows residuals for the baseline model; panel (c) showss residuals for the PEXRAV model; and panel d) shows residuals for the best-fit model with PEXRAV and a high-energy rollover (parameters for the best-fit model are listed in Table 5.

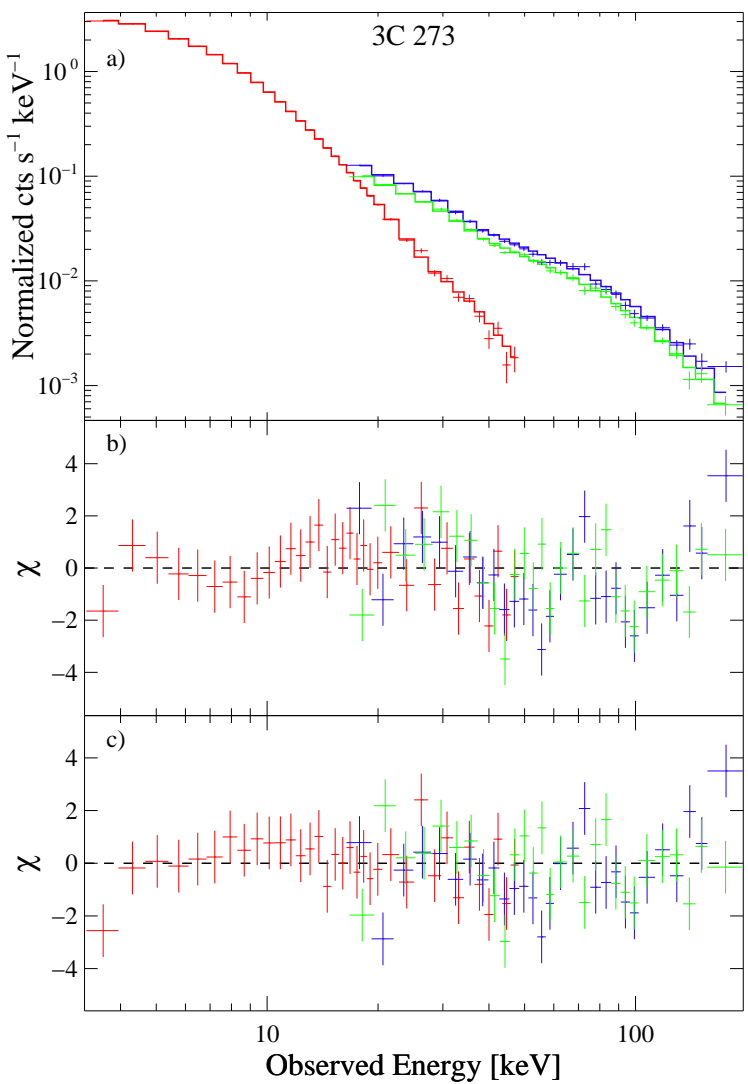

FIG. 25.- Data, model and data-model residuals for 3C 273. Panel (a) shows the PCA and HEXTE data along with the best-fit model (solid line); panel (b) shows residuals for the baseline model; and panel (c) shows residuals for the best-fit model (parameters for the best-fit model are listed in Table 4 ). 


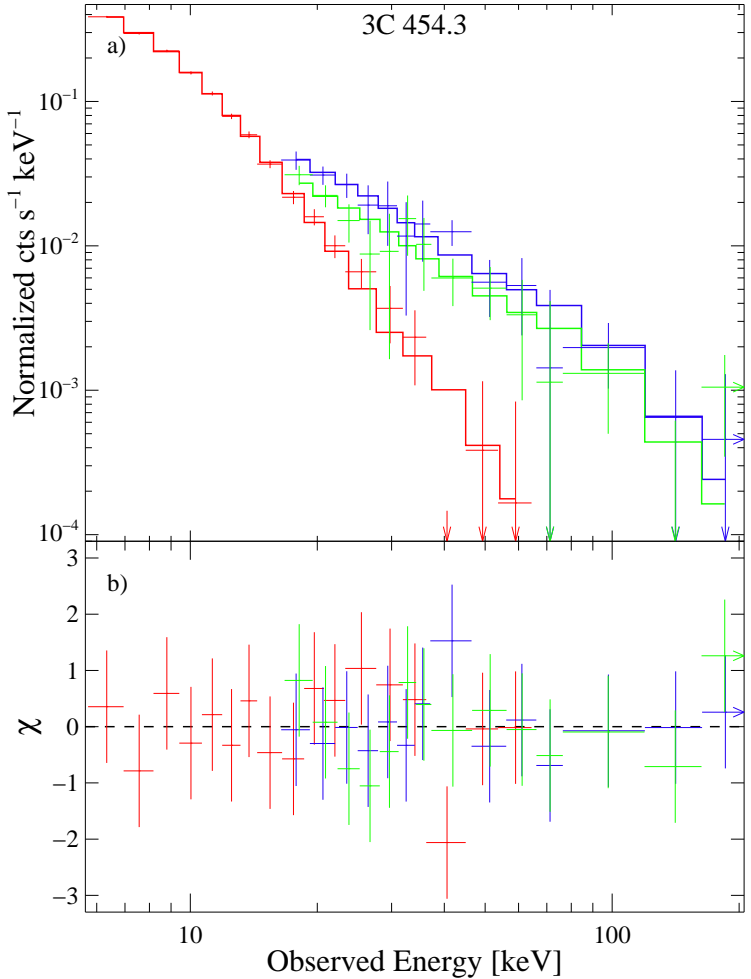

FIG. 26.- Data, model and data-model residuals for 3C 454.3. Panel (a) shows the PCA and HEXTE data along with the best-fit model (solid line); panel (b) shows residuals for the best-fit model (parameters are listed in Table 6.

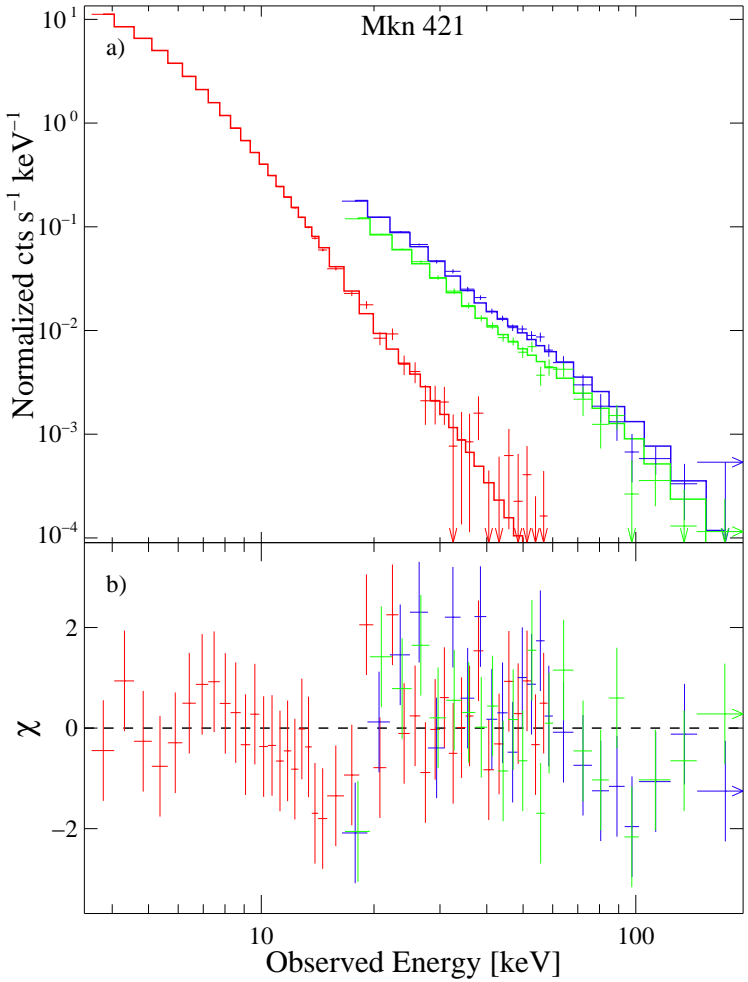

FIG. 27.- Data, model and data-model residuals for Mkn 421. Panel (a) shows the PCA and HEXTE data along with the best-fit model (solid line); panel (b) shows residuals for the best-fit model (parameters are listed in Table 6.

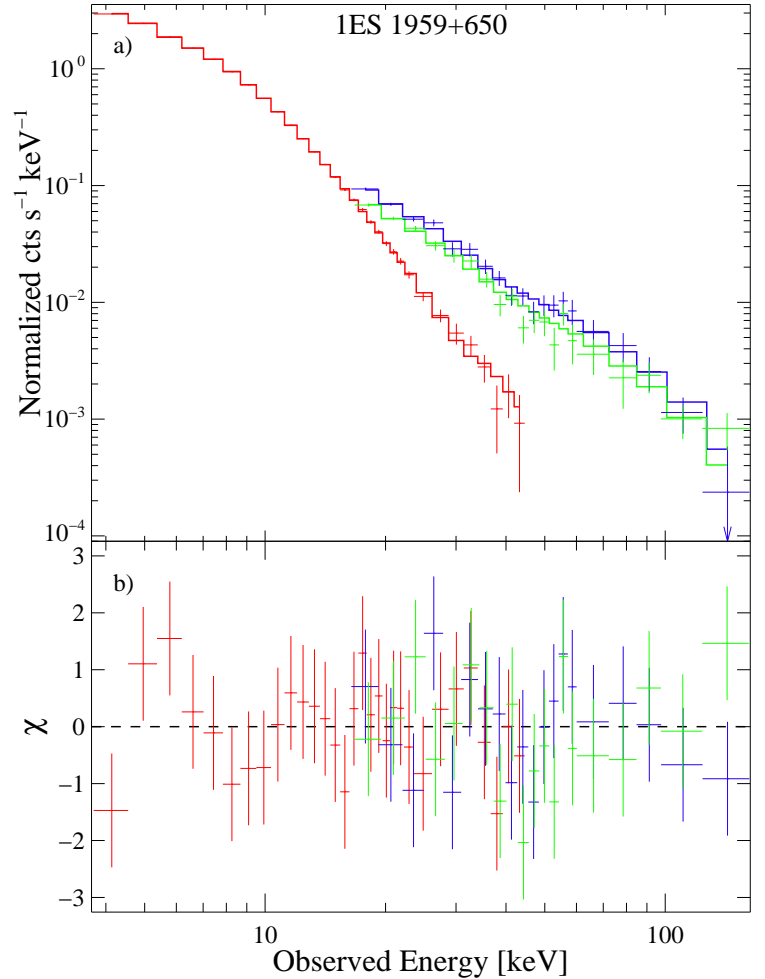

FIG. 28. - Data, model and data-model residuals for 1ES 1959+650. Panel (a) shows the PCA and HEXTE data along with the best-fit model (solid line); panel (b) shows residuals for the best-fit model (parameters are listed in Table 6).

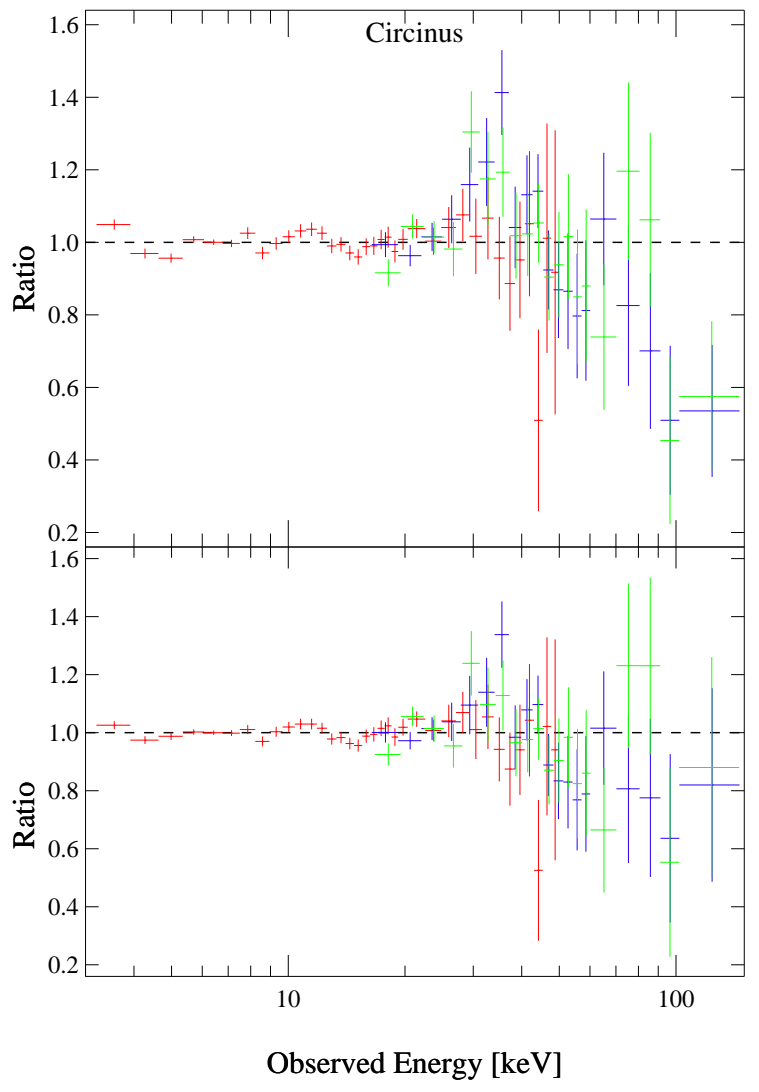

FIG. 29.- Data to model ratios for the best-fit reflection model (top) and best-fit rollover model (bottom). Parameters are given in Tables 4 and 5 respectively. 


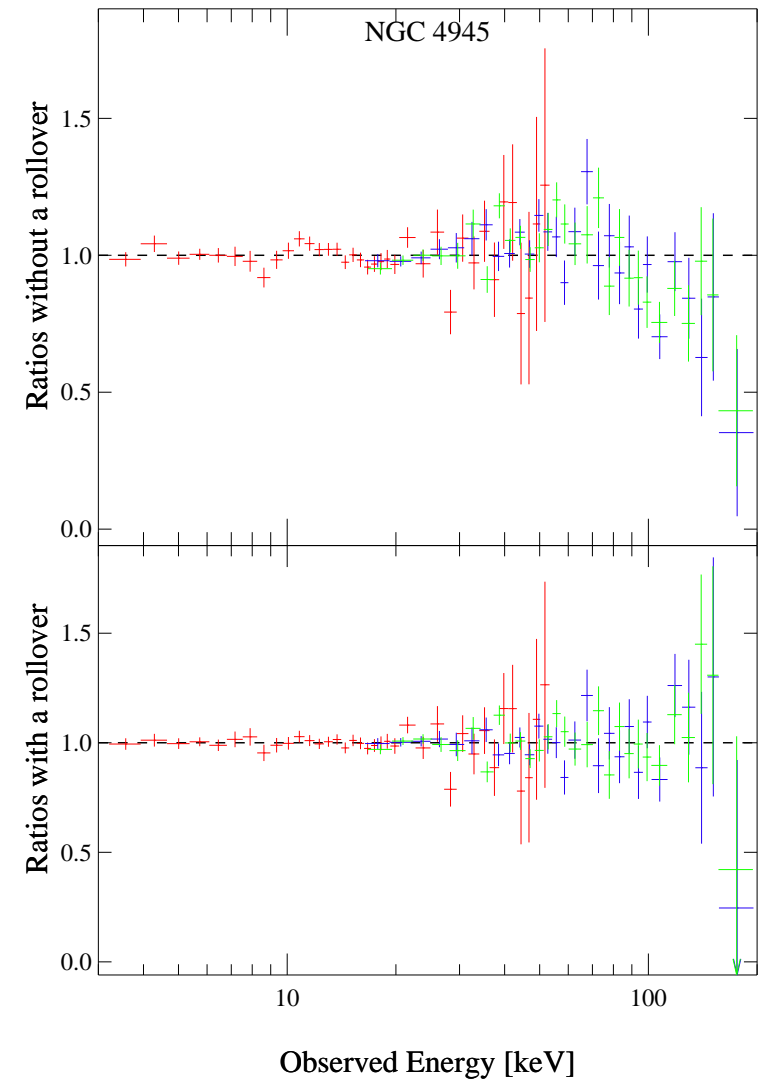

FIG. 30.- Data to model ratios for the best-fit reflection model (top) and best-fit rollover model (bottom). Parameters are given in Tables 4 and 5 respectively.

\subsection{Comparisons to Previous Surveys}

Most hard X-ray surveys of the extragalactic sky have concentrated on flux measurements and generation of luminosity functions, e.g. Piccinotti et al. (1982; HEAO-1-A2), Tueller et al. (2008; SwiftBAT), and Bassani et al. (2006; INTEGRAL-IBIS). Fits to spectra using non-simultaneous observations by various missions, e.g., Gondek et al. (1996; Ginga, CGRO-OSSE, EXOSAT, HEAO-1), Winter et al. (2008; Swift-BAT, XMM-Newton), and Molina et al. (2009; INTEGRAL-IBIS, XMM-Newton, Chandra, ASCA), have yielded interesting insights, but the lack of simultaneity and systematic uncertainties in cross-instrument normalizations has precluded definitive conclusions

The first X-ray spectral surveys of Seyfert AGN were compiled by Mushotzky et al. (1980), Rothschild et al. (1983), and Mushotzky (1984) using broadband data from $H E A O-1$-A2 and A4. Using mainly simple power-law or broken power-law models, these surveys established that the dominating X-ray continuum displayed a moderately narrow range of $\Gamma$. EXOSAT and Ginga observations established the necessity to model the Fe K $\alpha$ line (e.g., Nandra et al. 1989, Pounds et al. 1989). Nandra \& Pounds (1994) provided the next major spectral compilation, of 27 Seyferts observed by Ginga in the $1.5-37 \mathrm{keV}$ band. They found the need to model the Compton reflection hump and found a mean reflection strength of $\langle R\rangle=1.60 \pm 0.06$. They found a mean photon index of $\Gamma=1.95 \pm 0.05$ with a dispersion of $\sigma=0.15 \pm 0.04$ when reflection was included. They did not, however, have the advantage of sensitivity above $20 \mathrm{keV}$ with which to test for curvature beyond that of the reflection component.

A large spectral survey of Seyfert spectra above $50 \mathrm{keV}$ performed by Zdziarski et al. (2000) using CGRO-OSSE, determined photon indices in the $50-150 \mathrm{keV}$ band, although uncertainties were quite high $(15 \%-30 \%$ for $1 \sigma$ uncertainties) despite exposure times of $100 \mathrm{~s}-1000 \mathrm{~s}$ of ks. Of the 27 Seyfert galaxies observed by OSSE, 19 have measured power-law photon indices with mean values of $\Gamma=2.37 \pm 0.11$ for Seyfert 1's and $2.06 \pm 0.15$ for Seyfert 2's. When a cut-off power law was employed, the average values of $\Gamma$ dropped to $1.69_{-0.81}^{+0.57}$ and $1.33_{-0.52}^{+0.56}$, with exponential cut-off energies of $120_{-60}^{+220}$ $\mathrm{keV}$ and $130_{-50}^{+220} \mathrm{keV}$ respectively for Seyfert 1's and 2's. For com-
TABLE 7

FE LINE EQUiVALENT

WIDTHS FOR BEST-FIT

MODELS

\begin{tabular}{lc}
\hline \hline Source & EW $(\mathrm{eV})$ \\
\hline NGC 4151 & $110 \pm 20$ \\
IC 4329a & $130 \pm 30$ \\
NGC 3783 & $170 \pm 30$ \\
NGC 5548 & $150 \pm 30$ \\
Mkn 509 & $110 \pm 30$ \\
MR 2251-178 & $130 \pm 40$ \\
NGC 3516 & $150 \pm 30$ \\
NGC 3227 & $110 \pm 40$ \\
NGC 4593 & $230 \pm 20$ \\
NGC 7469 & $150 \pm 30$ \\
3C 111 & $180 \pm 60$ \\
3C 120 & $110 \pm 70$ \\
3C 273 & $40 \pm 20$ \\
Cen A & $90 \pm 10$ \\
NGC 5506 & $410 \pm 70$ \\
MCG-5-23-16 & $140 \pm 20$ \\
NGC 4507 & $180 \pm 30$ \\
Circinus & $2400 \pm 100$ \\
NGC 7582 & $330 \pm 90$ \\
NGC 4945 & $1600 \pm 500$
\end{tabular}

NoTE. - Fe line equivalent widths (EW) for all objects using the best fit model for each.

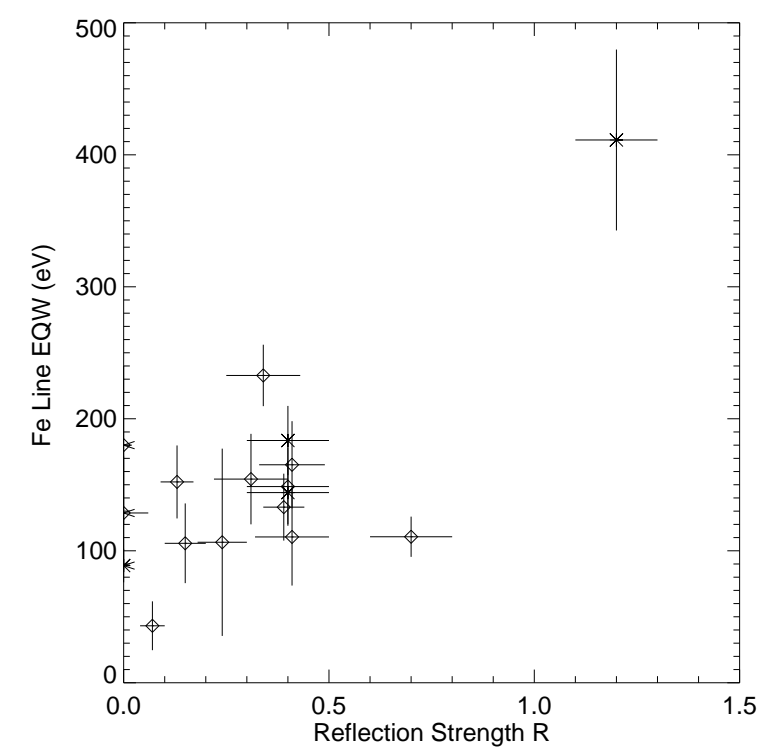

FIG. 31.- Fe line equivalent width versus $R$ for all Seyferts excluding NGC 7582 which has a poorly constrained $R$ value, and also Circinus and NGC 4945 which have unusually high Fe line equivalent widths due to extreme absorption of the power laws. Diamonds indicate Seyfert 1's, X's indicate Seyfert 2's.

parison, our results from the $20-100 \mathrm{keV}$ band only modeled with a simple power law gave an average values of $\Gamma=1.81$ for Seyfert 1's and $\Gamma=1.71$ for Seyfert 2's.

Unification schemes imply that Seyfert 1's and 2's should share a common range of photon indices, since according to this model only the amount of absorption distinguishes the different types of Seyferts while the intrinsic central engine is the same. Gondek et al. (1996), who created an average radio-quiet Seyfert 1 spectrum with Ginga+OSSE and EXOSAT+OSSE samples, found $\Gamma=1.90 \pm 0.05$, a rollover energy between 400 and $2700 \mathrm{keV}, R=0.76 \pm 0.15$, and an Fe line EW $=120 \pm 40 \mathrm{eV}$. Smith \& Done (1996) analyzed a Ginga 
sample of Seyfert 2's, finding mean $\Gamma=2.0$, very close to that of Seyfert $1 \mathrm{~s}$, and with similar dispersion, all of which is consistent with unification schemes. Our results for the power-law photon indices gave an average $\Gamma=1.79$ with a standard deviation of 0.09 for all Seyferts, $\Gamma=1.79$ with a standard deviation of 0.09 for Seyfert 1's and $\Gamma=1.81$ with a standard deviation of 0.08 for Seyfert 2's, consistent with previous results and with unification schemes.

Zdziarski et al. (1995) created an average Seyfert spectrum also from Ginga+OSSE data with rollovers near a few hundred keV. Perola et al. (2002) studied nine Seyfert 1's with BeppoSAX, measuring rollovers between 80 and $300 \mathrm{keV}$ with large error bars (up to 100's of keV). In contrast, our results for the high-energy rollover yielded mainly lower limits, with tentative detections for only 3/20 Seyferts, as discussed in Section 4.3.

The recent 22-month Swift-BAT survey (Tueller et al. 2010) contained all 23 of our selected sources covering an energy range 14-195 $\mathrm{keV}$, comparable to our HEXTE-only data. Photon indices derived from their hardness ratios were slightly steeper than those derived from a simple power-law fit to HEXTE data (see Table 2) by about $0.1-0.2$, probably due in part to differences in calibration for the two instruments (BAT calibration to the Crab used $\Gamma=2.15$ while HEXTE used a value of 2.062 ; Tueller et al. 2010 and Rothschild et al. 1998 respectively; Jourdain \& Roques 2009 used a broken power-law with $\Gamma=\sim 2.07$ below $100 \mathrm{keV}$ to model the Crab using the extensively calibrated SPI instrument aboard INTEGRAL-SPI). BAT spectral analysis is ongoing.

\subsection{The Compton Reflection Component}

$\mathrm{X}$-ray reflection of the illuminating power-law continuum is expected to occur at the surface of the optically thick, radiatively efficient, geometrically thin accretion disk whose thermal emission comprises the "big blue bump" in the optical/UV. Compton-thick material can also be present in the form of the putative homogeneous torus commonly invoked in classical Seyfert $1 / 2$ unification schemes and thought to be located $\sim 1$ pc from the black hole (e.g., Antonucci 1993); more recent models incorporate sub-pc scale Compton-thick clumps (e.g., Nenkova et al. 2008; Elitzur \& Shlosman 2006).

Constraining the level of reflection relative to that of the illuminating power-law can, in principle, yield information on the sky-covering fraction of the reflecting material (as seen from the X-ray continuum source, assumed to be the central X-ray corona), thereby providing constraints on how much Compton-thick material is in the form of a torus, disk, etc. In practice, however, this is not straightforward. The variability of the illuminating X-ray continuum (in both the photon index and normalization) and the fact that there is a non-zero light-travel time from the X-ray corona to the reflecting material can result in a lag between the properties of the observed power-law continuum and the properties of the power-law continuum illuminating the reflecting material.

A spectral survey such as ours, wherein we have used data spanning as long a baseline as possible, thus has an advantage compared to measurements obtained via single-epoch spectral fitting: there is a much higher likelihood that the long-term average normalizations and photon indices of the observed and illuminating power-laws will be equal, provided that the lag time between the variations in the continuum and the responses in the strength of the Compton reflection component (assumed to be dominated by the light travel time) is substantially less than the duration of the monitoring. For most of the objects in the current sample, this condition is satisfied provided that the bulk of the reflecting material in Seyferts lies within a few light-years of the black hole and corona. If the reflecting material is spatially extended, then rapid variations will be smoothed out anyway.

Moreover, an additional complication is the geometry of the reflecting material and its orientation relative to the observer. In this work, for simplicity, we used PEXRAV, which assumes a semi-infinite slab, most applicable to a "lamppost" geometry wherein the illuminating $\mathrm{X}$-ray source lies above an accretion disk. However, as discussed by Murphy \& Yaqoob (2009), PEXRAV does not account for transmission through the slab. In addition, as Compton scattering in the Thompson regime is an isotropic process, photons are preferentially scattered forward and backward (parallel and anti-parallel to the path of the incident photons). For a torus-shaped structure, whose inner face is preferentially illuminated by a central continuum source, photons will be preferentially scattered parallel to the equatorial plane instead of in a direction parallel to the rotation axis. For a torus with its equatorial plane oriented perpendicular to the observer, the amount of reflected emission reaching the observer will be relatively less. Meanwhile, in a face-on lamppost/disk geometry, the bulk of scattered photons will be parallel/anti-parallel to the line of sight. Consequently, for a given incident flux, the amount of reflected flux reaching the observer will be far less in the case of a torus than for a disk; this factor is $\sim 6$ for the reflected continuum near $6 \mathrm{keV}$ and is $\sim 3$ near $20 \mathrm{keV}$ (Murphy \& Yaqoob 2009; their Figure 7). Models for reflected emission from a torus geometry are still under development and can be applied to the current sample in future work.

In the current work, where we have used PEXRAV, we find a value of $\langle R\rangle \sim 0.35$ with a standard deviation of 0.16 for Seyfert 1's as a whole (excluding the FSRQ 3C 273 and objects that do not show evidence for a reflection hump) and $\langle R\rangle \sim 0.67$ with a standard deviation of 0.46 for the three Compton-thin Seyfert 2's (excluding Cen A), but we must rely on several assumptions in order to infer physically meaningful constraints on the geometry of the reflecting material for the cases of a lamppost/disk geometry or a centrally-illuminated torus. (For the remainder of this section, we omit the Compton-thick sources due to possible model degeneracy problems resulting from the spectral complexity and large systematic uncertainties on $R$ ). Angles of inclination have also been assumed for classes of objects for simplicity $\left(30^{\circ}\right.$ and $60^{\circ}$ for Seyfert 1's and Compton-thin Seyfert 2's, respectively) as opposed to relying on model-dependent methods such as relativistic Fe $\mathrm{K} \alpha$ line fitting, (e.g., Reeves et al. 2007), and using the inclination of the host galaxy as a proxy is not feasible since there is no guarantee that the planes of the accretion disk and galaxy are aligned.

Assuming that the lamppost/disk geometry and the PEXRAV modeling are valid, the covering fractions (as seen from the corona) implied by the above values of $\langle R\rangle$ are (0.35) $2 \pi$ sr for Seyfert 1's and (0.67) $2 \pi$ sr for Compton-thin Seyfert 2's. These values suggest several possibilities: the radiatively efficient disk may not extend down to the innermost stable orbit, may be too highly ionized at the inner radii to emit substantial amounts of reflected emission, and/or the entire disk may not be well illuminated due to self-shielding or the X-ray continuum emission being preferentially beamed away from the disk. If the inner disk is indeed very highly-ionized, then we would expect some line emission from highly-ionized Fe; however given the PCA resolution; that line flux may be overwhelmed by emission from neutral Fe. The majority of our energy centroid values for the Fe $\mathrm{K}$ lines ( 16 out of the 20 sources in which an Fe line is detected) are consistent with $6.4 \mathrm{keV}$, i.e., with the bulk of the Fe line photons originating in cold/neutral gas.

If instead all the reflected emission originates in a torus, the covering factors may be 3-6 times larger, suggesting that a very large fraction of the sky may be covered; one possibility is that the illuminated inner face of the torus may be substantially vertically extended. A more complex geometry (such as a distribution of Compton-thick clumps) of course cannot be excluded and indeed could explain how it is possible to obtain values of $R>1$ as in the case of NGC 5506.

Finally, combining knowledge of the Compton hump strength and the EW of the Fe K emission line can yield estimates of how much of the line emission originates in Compton-thick material as opposed to Compton-thin. A relativistically broadened $\mathrm{Fe} \mathrm{K} \alpha$ component indicates a radiatively-efficient disk (as opposed to a radiatively inefficient flow, e.g., Narayan et al. 1998); whereas if only a narrow $\mathrm{Fe}$ line component is confirmed, then the sites of Compton reflection are more likely in the distant torus.

For the lamppost/disk geometry if the disk is inclined at $30^{\circ}\left(60^{\circ}\right)$ to the line of sight and illuminated by a power-law with $\Gamma=1.8$, a value of $R=1$ corresponds to EW 125 (90) eV (George \& Fabian 1991; their Figure 14 with correction factor corresponding to the abundances of Wilms et al. 2000). We assume solar Fe abundance for purpose of this discussion. For our average values of $R$ for the Seyfert 1's, we therefore predict $\mathrm{EW}_{\text {disk }} \sim 40 \mathrm{eV}$. For the Comptonthin Seyfert 2's, $\mathrm{EW}_{\text {disk }} \sim 60 \mathrm{eV}$. Alternatively, for a torus geometry, assuming a continuum with $\Gamma=1.9$ illuminating gas with a column density of $2 \times 10^{24} \mathrm{~cm}^{-2}$, with the torus inclined by $30^{\circ}$; Murphy \& Yaqoob (2009; their Figure 9) gives similar values: $\mathrm{EW}_{\text {torus }} \sim 50 \mathrm{eV}$ for the Seyfert 1's and $\sim 40 \mathrm{eV}$ for the Compton-thin Seyfert 2's. 
TABLE 8

FLUXES AND LUMINOSITIES

\begin{tabular}{|c|c|c|c|c|c|}
\hline $\begin{array}{l}\text { Source } \\
\text { Name }\end{array}$ & $\begin{array}{l}F_{2-10} \\
\text { Observed } \\
\left(10^{-11} \mathrm{erg} \mathrm{cm}^{-2} \mathrm{~s}^{-1}\right)\end{array}$ & $\begin{array}{l}F_{20-100} \\
\text { Observed } \\
\left(10^{-11} \mathrm{erg} \mathrm{cm}^{-2} \mathrm{~s}^{-1}\right)\end{array}$ & $\begin{array}{l}L_{2-10}(\text { Rest Frame }) \\
\text { Power-law only } \\
\left(\mathrm{erg} \mathrm{s}^{-1}\right)\end{array}$ & $\begin{array}{l}L_{20-100} \text { (Rest Frame) } \\
\text { Power Law Only } \\
\left(\mathrm{erg} \mathrm{s}^{-1}\right)\end{array}$ & $\begin{array}{l}L_{\mathrm{CR}} / L_{(\mathrm{PL}+\mathrm{CR})} \\
\text { in } 20-100 \mathrm{keV} \\
\text { (Rest Frame) }\end{array}$ \\
\hline NGC 4151 & 16.92 & 43.48 & $5.14 \times 10^{43}$ & $4.64 \times 10^{42}$ & $39 \%$ \\
\hline IC 4329a & 13.97 & 19.37 & $7.42 \times 10^{43}$ & $7.65 \times 10^{43}$ & $26 \%$ \\
\hline NGC 3783 & 7.34 & 12.12 & $1.39 \times 10^{43}$ & $1.57 \times 10^{43}$ & $27 \%$ \\
\hline NGC 5548 & 5.97 & 8.57 & $3.88 \times 10^{43}$ & $4.94 \times 10^{43}$ & $12 \%$ \\
\hline Mkn 509 & 5.77 & 8.75 & $1.32 \times 10^{44}$ & $1.81 \times 10^{44}$ & $12 \%$ \\
\hline MR 2251-178 & 4.46 & 6.25 & $3.78 \times 10^{44}$ & $5.32 \times 10^{44}$ & $<4.6 \%$ \\
\hline NGC 3516 & 4.45 & 8.80 & $1.02 \times 10^{43}$ & $1.24 \times 10^{43}$ & $22 \%$ \\
\hline NGC 3227 & 4.11 & 7.49 & $2.34 \times 10^{42}$ & $2.84 \times 10^{42}$ & $27 \%$ \\
\hline NGC 4593 & 4.19 & 6.29 & $9.27 \times 10^{42}$ & $1.16 \times 10^{43}$ & $23 \%$ \\
\hline NGC 7469 & 3.33 & 4.53 & $1.47 \times 10^{43}$ & $1.57 \times 10^{43}$ & $26 \%$ \\
\hline 3C 111 & 5.21 & 8.66 & $2.73 \times 10^{44}$ & $4.27 \times 10^{44}$ & $<0.8 \%$ \\
\hline $3 \mathrm{C} 120$ & 5.30 & 7.52 & $1.16 \times 10^{44}$ & $1.40 \times 10^{44}$ & $18 \%$ \\
\hline Cen A & 28.42 & 66.01 & $8.59 \times 10^{41}$ & $1.08 \times 10^{42}$ & $<0.02 \%$ \\
\hline NGC 5506 & 10.65 & 16.88 & $1.01 \times 10^{43}$ & $9.70 \times 10^{42}$ & $42 \%$ \\
\hline MCG-5-23-16 & 9.60 & 16.00 & $2.03 \times 10^{43}$ & $2.35 \times 10^{43}$ & $21 \%$ \\
\hline NGC 4507 & 1.43 & 14.94 & $2.41 \times 10^{43}$ & $4.05 \times 10^{43}$ & $19 \%$ \\
\hline Circinus & 2.63 & 20.62 & $2.22 \times 10^{41}$ & $3.13 \times 10^{41}$ & $64 \%$ \\
\hline NGC 4945 & 0.61 & 19.89 & $1.15 \times 10^{41}$ & $4.02 \times 10^{41}$ & $<0.4 \%$ \\
\hline NGC 7582REFL & 1.04 & 5.62 & $6.03 \times 10^{41}$ & $7.24 \times 10^{41}$ & $75 \%$ \\
\hline NGC 7582PC & 1.01 & 6.54 & $3.09 \times 10^{42}$ & $3.47 \times 10^{42}$ & - \\
\hline $3 \mathrm{C} 273$ & 11.90 & 19.92 & $7.23 \times 10^{45}$ & $1.15 \times 10^{46}$ & $6 \%$ \\
\hline 3C 454.3 & 7.41 & 13.05 & $2.06 \times 10^{47}$ & $3.60 \times 10^{47}$ & - \\
\hline Mkn 421 & 40.00 & 10.14 & $8.63 \times 10^{44}$ & $2.18 \times 10^{44}$ & - \\
\hline 1ES $1959+650$ & 15.48 & 9.37 & $1.17 \times 10^{45}$ & $7.02 \times 10^{44}$ & - \\
\hline
\end{tabular}

Note. - Columns (2) and (3) list the observed frame, model-dependent 2-10 and 20-100 keV fluxes, not corrected for absorption, from each source's best-fit model. The 20-100 keV fluxes listed in col. (3) were determined from HEXTE responses, averaged between the two HEXTE clusters and weighted by exposure time and effective area. Column (4) lists the absorption corrected, rest-frame $2-10 \mathrm{keV}$ luminosity of the power-law continuum component only i.e., without the Fe line, Compton reflection hump, and after correcting for all cold (including Galactic) and warm absorbers. Isotropic emission was assumed (i.e., there is no correction for beaming effects in blazars). Similarly, column (5) lists the absorption corrected, rest-frame 20-100 keV luminosity determined using HEXTE responses, weighted by exposure time and effective area between the two clusters. Column (6) lists the ratio of the Compton reflection component emission to the total (power-law plus Compton reflection hump) emission in the rest-frame $20-100 \mathrm{keV}$ band for each source's best-fit model, again using HEXTE responses and weighted between HEXTE A and $\mathrm{B}$ by exposure time and effective area.

The average observed equivalent width $\left\langle\mathrm{EW}_{\mathrm{obs}}\right\rangle$ for just the Seyfert 1's (again, not including 3C 273, whose observed EW may be diluted due to contamination from the jet) is $145 \pm 35 \mathrm{eV}$, so we estimate that, on average, $\sim 25 \%-35 \%$ of the Fe line originates in Compton-thick material in Seyfert 1s. For the Compton-thin Seyfert 2's, we find $<$ $\left.\mathrm{EW}_{\mathrm{obs}}\right\rangle=205 \mathrm{eV}$, suggesting that, on average, $\sim 20 \%-30 \%$ of the $\mathrm{Fe}$ line originates in Compton-thick material in these objects.

Risaliti (2002) analyzed BeppoSAX data of Compton-thin Seyfert 2's, finding that on average the Fe line EW increased slightly when $N_{\mathrm{H}}$ was above $\sim 3 \times 10^{23}$. He concluded that this must mean there is contribution to the Fe line flux that does not come from the accretion disk (otherwise the Fe line would be just as absorbed as the continuum). We find a similar and far more dramatic increase in EW for our two heavily absorbed sources, Circinus and NGC 4945 (see Table 7). This confirms the findings of Risaliti (2002) and is consistent with the idea that a substantial amount of reflection/emission may come from within the torus.

Figure 31 shows the measured values of EW plotted against the Compton reflection strength In the reflection models discussed so far, one may expect a correlation between these parameters if the bulk of the Fe line originates in Compton-thick material. In this plot, we have omitted the Compton-thick sources. The Pearson correlation coefficient $r_{\mathrm{P}}=0.79$, with a null hypothesis probability (probability of obtaining the correlation by chance) $P_{r}=7 \times 10^{-4}$. NGC 5506 stands out as an outlier as it has the largest values of both $R$ and $\mathrm{EW}_{\mathrm{obs}}$ for the non-Compton-thick sources. When omitted from the correlation,
$r_{\mathrm{P}}$ falls to $0.28\left(P_{r}=0.35\right)$. The lack of a strong correlation between $R$ and $\mathrm{EW}$ is consistent with the notion above that it is very unlikely that the bulk of the Fe line emission originates in Compton-thick material. This is consistent with the findings of Risaliti (2002) who found no correlation between EW and $R$ (his Figure 5).

\subsection{High-Energy Rollovers}

High-energy rollovers in the power-law continua of Seyferts are expected if the power-law is generated via thermal Comptonization of soft seed photons, most likely blackbody emission from the accretion disk, (e.g., Haardt et al. 1994, Titarchuk 1994, Poutanen \& Svensson 1996, and references therein). In the simplest models, if all the electrons in the corona are at the same temperature $T_{\mathrm{e}}$, a high-energy continuum rollover can be expected in the spectrum near an energy $\sim 3$ times $k_{\mathrm{B}} T_{\mathrm{e}}$ (assuming an optical depth near unity).

Such rollovers have been detected in the X-ray spectra for many accreting Galactic black hole (GBH) systems (e.g., Takahashi et al. 2008; Wilms et al. 2006; Kalemci et al. 2005; Yamaoka et al. 2005; Frontera et al. 2001). As Seyferts are generally X-ray fainter than GBH systems, constraining rollovers in AGN has been observationally challenging, however for sources with $\Gamma<2$, rollovers are expected in order to prevent divergence of the total energy. In general, the rollovers modeled in BeppoSAX spectra of Seyferts reported in the literature span the range $\sim 50--400 \mathrm{keV}$ with uncertainties anywhere from tens to a few hundred keV (e.g., Petrucci et al. 2001; Perola et al. 2002; Quadrelli et al. 2003). In our RXTE sample, adding high- 
energy rollovers to our spectral models yielded mostly only lower limits to $E_{\text {roll }}$ of $\sim 200-300 \mathrm{keV}$. This is similar to the results of Risaliti (2002) who concluded that high-energy cutoffs must not be an ubiquitous property of Seyfert galaxies.

We detect a rollover in Circinus around $40 \mathrm{keV}$, and NGC 4945 around $70 \mathrm{keV}$, and, tentatively, in MR 2251-178 around $100 \mathrm{keV}$. Extra curvature in the data/model residuals when no rollover is modeled suggest that the rollovers detected in Circinus and NGC 4945 are not spurious. However, problems encountered with model degeneracies during fitting led to unexpectedly low (and likely unrealistic) values for the photon index $\Gamma_{\mathrm{HX}}$ of the absorbed hard X-ray power law in each case. Given the extreme amount of absorption and the requirement to model an additional power-law component below $\sim 10 \mathrm{keV}$ in each object, Circinus and NGC 4945 have two of the most complex spectra in our sample. Successfully deconvolving all components, and accurately constraining rollovers and Compton reflection strengths in these objects may be possible in the future via observations spanning a broad energy range and having sufficient energy resolution in the Fe K bandpass. For the purposes of this paper, we have simply presented the results of model fits with and without rollovers, with the understanding for the reader that for the models with rollovers, bestfit values may have large systematic uncertainties.

For objects in which a rollover was not detected, we can be reasonably certain that the quality of our data precludes rollover energies below $\sim 100 \mathrm{keV}$. Excluding the blazars, 3C 273, and Cen A (each of which has X-ray emission dominated by or likely contaminated by X-ray emission from a relativistic jet), the lower limits on $E_{\text {roll }}$ for our objects range from 120 to $350 \mathrm{keV}$, with an average lower limit of $225 \mathrm{keV}$, which is very close to the upper limit of the HEXTE energy range. This would suggest that, on average, the electron temperatures in our Seyfert coronae must be at least $\gtrsim 75 \mathrm{keV}$.

The potential bias produced by our selection criteria may have had an adverse effect on the likelihood of finding rollovers below $100 \mathrm{keV}$ in our sample since our goal was to have a $3 \sigma$ detection at $100 \mathrm{keV}$. In addition, as thermal Comptonization is thought to be at work in both Seyfert and GBH coronae, then if there exists a relation between $\Gamma$ and $E_{\text {roll }}$ in Seyferts as seen in GBHs (Yamaoka et al. 2005), then our tendency to select flat-spectrum objects may be another source of bias against finding relatively low values of $E_{\text {rol }}$.

\section{CONCLUSION}

Thanks to the combination of the longevity of the RXTE mission, the sustained monitoring campaigns on many AGN, and the simultaneous operation of the PCA and HEXTE instruments, we have been able to extract broadband ( 3 to $\geq 100 \mathrm{keV}$ ) X-ray spectra with long integration times and moderately good spectral resolution. Although the HEXTE instrument is sometimes overlooked due to its low sensitivity on short timescales, we have demonstrated that HEXTE can indeed yield spectra out to at least $100 \mathrm{keV}$ for 23 AGN with a sufficient combination of brightness and exposure time. The fact that the spectra presented here are long-term averaged spectra means the ambiguity inherent in single-epoch spectral fitting caused by variability of the source is eliminated. In summary, we have presented the first high-quality broadband spectra sample with which to study the long-term average properties of X-ray bright Seyferts in the $>10 \mathrm{keV}$ sky.

Observed fluxes and unabsorbed power-law luminosities for the 210 and the $20-100 \mathrm{keV}$ range are given in Table 8 , along with the modeled fraction of reflected flux in the $20-100 \mathrm{keV}$ band. As these quantities are long-term averages in nearly all cases, they can be used by the community, e.g., for purposes of planning future observations of these objects, or for constraining models which seek to determine the contributions of low- $z$ Seyferts to the observed cosmic X-ray background $(\mathrm{CXB})$ and to the accretion history of the universe.

Our long-term averaged values for $\Gamma$ were generally consistent with previous results for individual objects; our averages by AGN classification were also consistent with previous surveys. Unsurprisingly, we found that the Fe line complex was necessary to model in all Seyfert spectra. Using a single Gaussian to model this component we found typical equivalent widths around 100-200 eV, roughly consistent with previous results. In contrast to the Fe line, Compton reflection components have not been well studied as, historically, the $>20$ sky has not been as well observed as the $2-10 \mathrm{keV}$ band. For our sample, we found that the Compton reflection component was required in the model for a vast majority of the Seyferts studied, again consistent with previous results such as Nandra \& Pounds (1994). Using the PEXRAV model in XSPEC, we find a average reflection fraction of $\sim 0.3 / 0.7$ in Seyfert $1 / 2$ 's coming from either a truncated disk or a torus. Assuming solar $\mathrm{Fe}$ abundance, only $25 \%-35 \%$ of the Fe line emission comes from Compton-thick material with the remainder arising in Comptonthin gas. We found tentative evidence for high-energy rollovers in the spectra of only three objects, Circinus, NGC 4945, and MR 2251178; we obtained lower limits of very roughly $100 \mathrm{keV}$ for the remaining objects implying $\mathrm{kT} \gtrsim 75 \mathrm{keV}$ in thermal Comptonization models.

The time-averaged model fits in this paper may be used in future time- or flux-resolved analyses of RXTE monitoring data to test for variations in the absorbing or reflecting components to constrain their locations and/or distributions. We conclude by commenting that future in-depth study of targets whose spectra require highly complex model fits, such as Circinus and NGC 4945, can yield fewer model degeneracies and better constraints on $\Gamma, E_{\text {roll }}$, etc., provided that observations are made with instruments featuring a combination of CCD-like (or better) energy resolution and broad energy coverage up to at least hundreds of $\mathrm{keV}$. Such observations are also required for further progress in better constraining the high-energy rollovers in Seyferts and understanding the properties of the X-ray corona, as well as allowing for testing of the newest Compton reflection models (e.g., Murphy \& Yaqoob 2009).

This research has made use of data obtained from the RXTE satellite, a NASA space mission. The authors wish to thank J. Wilms for reading the manuscript and providing helpful suggestions to guide the paper. This work has made use of HEASARC online services, supported by NASA/GSFC, and the NASA/IPAC Extragalactic Database, operated by JPL/California Institute of Technology under contract with NASA. The research was supported by NASA Constract NAS 5-30720 and Grant NNX09AG79G.

\section{REFERENCES}

Alexander, D.M., et al. 2003, ApJ, 125, 383

Antonucci R., 1993, ARA\&A, 31, 473

Arévalo, P., Uttley, P., Kaspi, S., Breedt, E., Lira, P. \& McHardy, I.M. 2008, MNRAS, 389, 1479

Arévalo, P., Uttley, P., Lira, P., Breedt, E., McHardy, I.M. \& Churazov, E. 2009, MNRAS, 397, 2004

Bania, T.M., Marscher, A.P. \& Barvainis, R. 1991, AJ, 101, 2147

Bassani, L., et al. 2006, ApJ, 636, 65

Beckmann, V., Wolter, A., Celotti, A., Costamante, L., Ghisellini, G., Maccacaro, T. \& Tagliaferri, G. 2002, A\&A, 383, 410

Benlloch, S., et al., 2001, A\&A, 371, 858

Bianchi, S., Balestra, I., Matt, G., Guainazzi, M. \& Perola, G.C. 2003, A\&A, 402, 141

Bianchi, S., Chiaberge, M., Piconcelli, E. \& Guainazzi, M. 2007, MNRAS, 374,697

${ }^{4}$ See also Petrucci et al. (2001) for investigations into a possible correlation between $\Gamma$ and corona temperature in Seyferts.
Bianchi, S., Matt., G., Balestra, I., Guainazzi, M.\& Perola, G.C. 2004, A\&A, 422, 65

Bianchi, S., Piconcelli, E., Chiaberge, M., Jiménez Bailón, Matt., G. \& Fiore, F. 2009, ApJ, 695, 781

Bird, A.J., et al., 2007, ApJS, 170, 175

Błażejowski, M., et al., 2005, ApJ, 630, 130

Blustin, A.J., et al., 2007, A\&A, 466, 107

Braito, V., et al., 2007, ApJ, 670, 978

Brenneman, L., Reynolds, C.S., Wilms, J. \& Kaiser, M.E. 2007, ApJ, 666, 817

Cappi, M., et al. 2006, A\&A, 446, 459

Chatterjee, R., Marscher, A.P., Jorstad, S.G., et al., 2009, ApJ 704, 1689

Chatterjee, R., et al. 2011, ApJ, Submitted

Cui, W. 2004, ApJ, 605, 662

De Rosa, A., Fabian, A.\& Piro, L., 2002, MNRAS, 334, L21

De Rosa, A., Piro, L., Fiore, F., Grandi, P., Maraschi, L., Matt, G., Nicastro, F. \& Petrucci, P.-O. 2002, A\&A, 387, 838

De Rosa, A., Piro, L., Matt, G.\& Perola, G.C. 2004 A\&A, 413, 895

De Rosa, A., et al. 2007, A\&A, 463, 912 
Dewangan, G.C. \& Griffiths, R.E. 2005, ApJ, 625, L31

Donato, D., Sambruna, R.M., Gliozzi, M. 2005, A\&A, 433, 1163

Done, C., Madejski, G.M. \& Smith, D.A. 1996, ApJ, 463, L63

Edelson, R. \& Nandra, K. 1999, ApJ, 514, 682

Elitzur, M. \& Shlosman, I. 2006, ApJ, 648, L101

Eracleous, M., Sambruna, R.M. \& Mushotzky, R.F. 2000, ApJ, 537, 654

Foschini, L., et al., 2006, A\&A, 453, 829

Frontera, F., et al. 2001, ApJ, 546, 1027

Fürst, F., Wilms, J., Rothschild, R., Pottschmidt, K., Smith, D.M. \& Lingenfelter, R., 2009, Earth \& Planet Sci. Lett., 281, 125

George, I.M. \& Fabian, A.C. 1991, MNRAS, 249, 352

Ghisellini, G., Foschini, L., Tavecchio, F., \& Pian, E., 2007, MNRAS, 382L, 82

Giebels, B., Dubus, G. \& Khélifi, B. 2007, A\&A, 462, 29

Giebels, B., et al., 2002, ApJ, 571, 763

Gliozzi, M., Sambruna, R.M. \& Eracleous, M. 2003, ApJ, 584, 176

Gondek, D., et al. 1996, MNRAS, 282, 646

Grandi, P., Guainazzi, M., Mineo, T., et al., 1997, A\&A, 325, L17

Grandi, P., Malaguti, G. \& Fiocchi, M. 2006, ApJ, 642, 113

Gruber, D.E., Blanco, P.R., Heindl, W.A., Pelling, M.R., Rothschild, R.E., \& Hink, P.L., 1996 A\&AS, 120C, 641

Gruber, D.E., Jung, G.V., \& Matteson, J.L. 1989, in AIPC, 186, 232

Grupe, D., Beuermann, K., Mannheim, K., \& Thomas, H.-C., 1999, A\&A, 350,805

Guainazzi, M., Matt, G., Brandt, W.N., Antonelli, L.A., Barr, P. \& Bassani, L. 2000, A\&A, 356, 463

Guainazzi, M., et al., 1999, A\&A, 346, 407

Gutierrez, K., et al., 2006, ApJ, 644, 742

Haardt, F., Maraschi, L. \& Ghisellini, G., 1994, ApJ, 432, 95

Isobe, N., Kubota, A., Makishima, K., Gandhi, P., Griffiths, R.E., Dewangen, G.C., Itoh, T. \& Mizuno, T. 2008, PASJ, 60S, 241

Itoh, T., et al., 2008, PASJ, 60S, 251

Jahoda, K., Markwardt, C.B., Radeva, Y., Rots, A.H., Stark, M.J., Swank, J.H., Strohmayer, T.E., \& Zhang, W., 2006, ApJS, 163, 401

Jorstad, S.G., et al., 2010, ApJ, 715, 362

Jourdain, E. \& Roques, J.P., 2009, ApJ, 704, 17

Kalberla, P.M.W. et al. 2005, A\&A, 440, 775

Kalemci, E., Tomsick, J.A., Buxton, M.M., Rothschild, R.E., Pottschmidt, K., Corbel, S., Brocksopp, C., \& Kaaret, P., 2005, ApJ, 622, 508

Kaspi, S., et al. 2004, ApJ, 611, 68

Kataoka, J., Tanihata, C., Kawai, N., Takahara, F., Takahashi, T., Edwards, P.G. \& Makino, F. 2002, MNRAS, 336, 932

Kataoka, J., et al., 2007, PASJ, 59, 279

Kinzer, R.L., et al. 1995, ApJ, 449, 105

Krawczynski, H., et al., 2004, ApJ, 601, 151

Lamer, G., Uttley, P. \& McHardy, I.M. 2000, MNRAS, 349, 919

Lamer, G., Uttley, P. \& McHardy, I.M. 2003, MNRAS, 342, L41

Lewis, K.T., Eracleous, M., Gliozzi, M., Sambruna, R.M. \& Mushotzky, R.F. 2005, ApJ, 622, 816

Liu, Y., et al., 2010, ApJ, 710, 1228

Madejski, G., Życki, P., Done, C., Valinia, A., Blanco, P., Rothschild, R.E. \& Turke, B. 2000, ApJ, 535, L87

Maoz, D., Markowitz, A., Edelson, R. \& Nandra K. 2002, AJ, 124, 1988

Magdziarz, P. \& Zdziarski, A., 1995, MNRAS, 273, 837

Markowitz, A. 2010, ApJ, 724, 26

Markowitz, A. 2009, ApJ, 698, 1740

Markowitz, A. \& Edelson, R. 2004, ApJ, 617, 939

Markowitz, A., Edelson, R. \& Vaughan, S. 2003a, ApJ, 598, 935

Markowitz, A. \& Reeves, J.N. 2009, ApJ, 705, 496

Markowitz, A., Reeves, J.N. \& Braito, V. 2006, ApJ, 646, 783

Markowitz, A., et al., 2003b, ApJ, 593, 96

Markowitz, A., et al. 2007, ApJ, 665, 209

Markowitz, A., et al. 2008, PASJ, 60, S277

Markowitz, A., et al. 2009, ApJ, 691, 922

Marshall, K., Ryle, W.T., \& Miller, H.R., 2008, ApJ, 677, 880

Marshall, K., Ryle, W.T., Miller, H.R., Marscher, A.P., Jorstad, S.G., Chicka,

B. \& McHardy, I.M., 2009, ApJ, 696, 601

Matt, G. et al. 1999 A\&A, 341, L39

M'Hardy, I.M. \& Uttley, P. 2005, MNRAS, 363, 586

McKernan, B., Yaqoob, T., George, I.M. \& Turner, T.J. 2003, ApJ, 593, 142

McKernan, B., Yaqoob, T. \& Reynolds, C.S. 2007, MNRAS, 379, 1359

Molina, M., et al. 2009, MNRAS, 399, 1293

Mueller, M., Madejski, G., Done, C., \& Zycki, P.T., 2004, AIPC, 714, 190

Murphy, K.D. \& Yaqoob, T. 2009, MNRAS, 397, 1549

Mushotzky, R.F. 1984, AdSpR, 3, 157

Mushotzky, R.F., Marshall, F.E., Boldt, E.A., Holt, S.S. \& Serlemitsos, P.J. 1980, ApJ, 235, 377

Nandra, K. 2006, MNRAS, 368, 62

Nandra, K., Le, T., George, I.M., Edelson, R.A., Mushotzky, R.F., Peterson, B.M. \& Turner, T.J. 2000, ApJ, 544, 734

Nandra, K., O’Neill, P.M., George, I.M. \& Reeves, J.N., 2007, MNRAS, 382, 194

Nandra, K. \& Papadakis, I. 2001, ApJ, 554, 710

Nandra, K. \& Pounds, K. A., 1994, MNRAS, 268, 405

Nandra, K., Pounds, K.A., Stewart, G.C., Fabian, A.C. \& Rees, M.J. 1989, MNRAS, 236, 39

Narayan, R., Mahadevan, R., \& Quataert, E. 1998, Theory of Black Hole Accretion Disks, ed. M.A. Abramowicz, G. Bjornsson, \& J.E. Pringle (Cambridge: Cambridge Univ. Press), 148
Nenkova, M. et al. 2008, ApJ, 685, 160

Netzer, H. et al. 2003, ApJ, 599, 933

Ogle, P.M., Marshall, H.L., Lee, J.C., \& Canizares, C.R., 2000, ApJ, 545, L81

Orr, A., Barr., P., Guainazzi, M., Parmar, A.N. \& Young, A.J. 2001, A\&A, 376, 413

Perola, G.C., Matt, G., Cappi, M., Fiore, F., Guainazzi, M., Maraschi, L., Petrucci, P.-O. \& Piro, L., 2002, A\&A, 389, 902

Petrucci, P.O. et al. 2001, ApJ, 556, 716

Pian, E., et al., 2006, A\&A, 449L, 21

Piccinotti, G., Mushotzky, R.F., Boldt, E.A., Holt, S.S., Marshall, F.E., Serlemitsos, P.J. \& Shafer, R.A. 1982, ApJ, 253, 485

Pottschmidt, K., et al. 2003, A\&A, 407, 103

Pucetti, S., Fiore, F., Risaliti, G., Capalbi, M., Elvis, M. \& Nicastro, F. 2007, MNRAS, 377, 607

Pounds, K.A., Nandra, K., Stewart, G.C. \& Leighly, K. 1989, MNRAS, 240, 769

Poutanen, J. \& Svensson, R. 1996, ApJ, 470, 249

Puccetti, S., Fiore, F., Risaliti, G., Capalbi, M., Elvis, M., \& Nicastro, F. 2007, MNRAS, 377, 607

Quadrelli, A., Malizia, A., Bassani, L., \& Malaguti, G. 2003, A\&A, 411, 77

Rebillot, P.F., et al. 2006, ApJ, 641, 740

Reeves, J.N., Nandra, K., George, I.M., Pounds, K.A., Turner, T.J. \& Yaqoob, T. 2004, ApJ, 602, 648

Reeves, J.N., et al. 2007, PASJ, 59S, 301

Reynolds, C.S., 1997, MNRAS, 286, 513

Reynolds, C.S., Iwasawa, K., Crawford, C.S. \& Fabian, A.C. 1998, MNRAS 299, 410

Risaliti, G. 2002, A\&A, 386, 379

Rothschild, R., Markowitz, A., Rivers, E., Suchy, S., Pottschmidt, K., Kadler, M., Müller, C., \& Wilms, J. 2011, ApJ, Submitted

Rothschild, R.E., Mushotzky, R.F., Baity, W.A., Gruber, D.E., Matteson, J.L. \& Peterson, L.E. 1983, ApJ, 269, 423

Rothschild, R.E., et al. 1998, ApJ, 496, 538

Rothschild, R.E., et al., 2006, ApJ, 641, 801

Sambruna, R.M. et al., 2001, ApJ, 546, L13

Schurch N.J., Warwick, R.S., Griffiths, R.E., Sembay, S., Ptak, A.F., 2003 MNRAS, 345, 423

Sidoli, L., et al., 2009, ApJ, 690, 120

Smith, D.A. \& Done, C. 1996, MNRAS, 280, 355

Smith, R.A.N., Page, M.J. \& Branduardi-Raymont, G. 2007, A\&A, 461, 135

Steenbrugge, K.C., Kaastra, J.S., Sako, M., Branduardi-Raymont, G., Behar,

E., Paerels, F.B.S., Blustin, A.J. \& Kahn, S.M. 2005a, A\&A, 432, 453

Steenbrugge, K.C., et al. 2005b, A\&A, 434, 569

Steinle, H., et al. 1998, A\&A, 330, 97

Summons, D.P., Arévalo, P., McHardy, I.M., Uttley, P. \& Bhaskar, A. 2007, MNRAS, 378, 649

Takahashi, H., et al., 2008, PASJ, 60S, 69

Tavecchio, F. et al. 2002, ApJ, 575, 137

Titarchuk, L., 1994, ApJ, 434, 570

Tueller, J., et al., 2010, ApJS, 186, 378

Tueller, J., et al. 2008, ApJ, 681, 113

Turner, T.J., Kraemer, S.B., George, I.M., Reeves, J.N. \& Bottorff, M.C. 2005 , ApJ, 618, 155

Turner, T.J., Perola, G.C., Fiore, F., Matt, G., George, I.M., Piro, L. \& Bassani, L. 2000, ApJ, 531, 245

Turner, T.J., Reeves, J.N., Kraemer, S.B. \& Miller, L. 2008, A\&A, 483, 161

Uttley, P., Edelson, R., McHardy, I.M., Peterson, B.M. \& Markowitz, A. 2003, ApJ, 584, L53

Vaughan, S., Edelson, R., Warwick, R.S., Malkan, M.A., \& Goad, M.R., 2001, MNRAS, 327, 673

Verner, D. A., Ferland, G. J., Korista, K. T., \& Yakovlev, D. G. 1996, ApJS 465,487

Weaver, K., Krolik, J.H. \& Pier, E.A. 1998, ApJ, 498, 213

White, N.E., Giommi. P. \& Angelini, L. 2000 VizieR On-line Data Catalog, 9031, 0

Wilms, J., Allen, A., \& McCray, M. 2000, ApJ 542, 914

Wilms, J., Nowak, M.A., Pottschmidt, K., Pooley, G.G., \& Fritz, S., 2006, A\&A, 447, 245

Winter, L.M., Mushotzky, R.F. \& Reynolds, C.S. 2006, ApJ, 649, 730

Winter, L.M., Mushotzky, R.F., Tueller, J. \& Markwardt, C. 2008, ApJ, 674, 686

Woźniak, P.R., Zdziarski, A.A., Smith, D., Madejski, G.M. \& Johnson, W.N. 2998, MNRAS, 299, 449

Xu, D.W., Komossa, S., Wei, J.Y., Qian, Y., \& Zhen, X.Z., 2003, ApJ, 590, 73

Yamaoka, K., Uzawa, M., Arai, M., Yamazaki, T., \& Yoshida, A. 2005, ChJAS, 5,273

Yaqoob, T. \& Padmanabhan, U. 2004, ApJ, 604, 63

Zdziarski, A. \& Grandi, P. 2001, ApJ, 551, 186

Zdziarski, A.A., Johnson, W.N., Done, C., Smith, D. \& McNaron-Brown, K. 1995, ApJ, 438, L63

Zdziarski, A.A., Johnson, W.N. \& Magdziarz, P. 1996, MNRAS, 283, 193

Zdziarski, A.A., Leighly, K.M., Matsuoka, M., Cappi, M. \& Mihara, T. 2002, ApJ, 573, 505

Zdziarski, A.A., Poutanen, J. \& Johnson, W.N. 2000, ApJ, 542, 703 


\section{A. NOTES ON INDIVIDUAL SOURCES}

In this section, we include notes pertaining to model fitting of each object's spectrum, and discuss spectral complexities such as partial covering and warm absorbers. Given the energy resolution of the PCA, all warm absorber velocity offsets relative to systemic were kept fixed. Since many warm absorber parameters are determined via gratings observations and/or using the soft X-ray band, ionization parameters and column densities were kept fixed.

We also compare our best-fit model parameters to those obtained by previous investigations of broadband X-ray spectra using RXTE, Suzaku, and BeppoSAX. We concentrate on continuum model parameters such as Compton reflection strengths and high-energy rollovers. Comparing our spectral model results to previous investigations is not always straight forward, as different broadband models may have been used and the source may display spectral variability in addition to flux variability. Furthermore, model degeneracies may exist between certain parameters, such as $\Gamma$ and $R$, or $\Gamma$ and $E_{\text {roll }}$. Below, we have tried to identify $90 \%$ uncertainties in the literature.

\section{NGC 4151}

RXTE PCA spectral data for NGC 4151 taken before 2001 February were published by Markowitz et al. (2003a); however, we include additional data up to 2004 June as well as all available HEXTE data. As one of the brightest Seyferts, NGC 4151 has been studied by every major X-ray mission to date, and is known to have a rather complex absorbed spectrum. Indeed, fitting a simple model consisting of a powerlaw with no absorption other than the Galactic column produced a very poor fit $\left(\chi^{2} / \mathrm{dof}=10324 / 86\right.$ with a very flat power-law photon index, $\Gamma \sim 1.2$ ), and allowing full-covering absorption by cold material in excess of the Galactic column offered a better, though still not quite poor fit $\left(\chi^{2} / \mathrm{dof}=3408 / 85\right)$.

We applied a partial-covering cold absorber using ZPHABS following DeRosa et al. (2007), who analyzed a series of BeppoSAX observations of this source. $\chi^{2} /$ dof fell to $766 / 84$ for $N_{\mathrm{H}} \sim 9 \times 10^{23} \mathrm{~cm}^{-2}$ and covering fraction $f_{\mathrm{c}} \sim 70 \%$, but there remained very large residuals below 10 $\mathrm{keV}$.

Following Schurch et al. (2003), who analyzed XMM-Newton observations obtained in 2000, we then applied an additional full-covering cold absorber, with column density fixed at $3.4 \times 10^{22} \mathrm{~cm}^{-2}$ (see also Ogle et al. 2000 and Zdziarski et al. 2002). DeRosa et al. (2007) modeled this component to be mildly ionized, with $\log (\xi) \sim 0-1$, but modeling this component as cold gas was adequate to yield a good fit in our best-fit models. This yielded our baseline model fit, with $\chi^{2} / \mathrm{dof}=682 / 83$. The best-fit parameters are listed in Table 3 . We kept the column density of the full-covering gas as a fixed parameter: thawing it caused several model parameters to deviate wildly from expected values based on previous observations, and after adding the Compton reflection component, thawing this column did not yield any further improvement in the fit.

After adding the Compton reflection component (see Table 4) our best-fit values for the column density and covering fraction for the partial covering absorber are broadly consistent with values found by Zdziarski et al. (2002) (for a 0.4-400 keV spectrum from near-simultaneous ASCA and OSSE spectra) and DeRosa et al. (2007). Our best-fit values for the covering fraction are within the ranges given by DeRosa et al. (2007) and Pucetti et al. (2007).

Values of the Compton reflection strength $R$ in the literature when data $>10 \mathrm{keV}$ are included are generally $\lesssim 1$, and a variety of broadband models have been used to model the complex absorption below $10 \mathrm{keV}$, affecting values of $\Gamma$. Given the degeneracy between $\Gamma$ and $R$, a comparison between our best-fit value of $R$ and those in the literature is therefore not straightforward. Furthermore, De Rosa et al. (2007) claim that the Compton hump was detected in 1996 with $R \sim 2$ but disappeared by 2000/2001, so this component's normalization relative to that of the power law may be time variable.

Adding a high-energy rollover, we found that $\chi^{2} /$ dof fell to $132 / 82$, for $E_{\text {roll }}=156_{-20}^{+26} \mathrm{keV}$, with $\Gamma$ flattening to $1.65 \pm 0.04$ (the best-fit values for the partial covering component $N_{\mathrm{H}}$ and $f_{\mathrm{c}}$ were $5.6 \pm 0.4 \times 10^{23} \mathrm{~cm}^{-2}$ and $52 \%$, respectively). However, as seen in Figure 6 there is a substantial "dip" near $100 \mathrm{keV}$. This is most likely a spurious feature related to background subtraction, as above this energy the background spectrum flattens and the source flux falls with increasing energy to below $1 \%$ of the background. The majority of the decrease in $\chi^{2}$ when a high-energy rollover is added is attributed to "fitting" this $100 \mathrm{keV}$ dip, and so we do not believe that the evidence for a rollover is robust in this object. We adopt a lower limit to $E_{\text {roll }}$ of $136 \mathrm{keV}$, consistent with Zdziarski et al. (2002) and most spectral fit results in DeRosa et al. (2007).

Analysis of a long-term, time-averaged spectrum assumes that the form of the spectral model is valid on shorter time-scales. Puccetti et al. (2007) demonstrated that NGC 4151 exhibits variations in the properties of its absorber on both rapid (time scales of tens of ks) and long (years) time scales. They attributed the variations to transits along the line of sight by gas clouds consistent with an origin in the broad line region. However, the magnitude of the variations (factors of $2-5$ in the column densities of the full-covering and partial covering absorbers and factors of $\sim 20 \%-30 \%$ in covering fraction) is likely small enough not to invalidate the above assumption for our long-term spectral analysis.

\section{IC $4329 a$}

Previous PCA + HEXTE joint spectral fits were published by Markowitz, Reeves \& Braito (2006). They included data only from 2003 April through 2005 October, while here we include data up through 2007 August plus data from three earlier campaigns.

Following Steenbrugge et al. (2005b), we included a cold absorber with a column density $N_{\mathrm{H}}$ fixed at $1.7 \times 10^{21} \mathrm{~cm}^{-2}$ and two warm absorber zones, one with $\log (\xi)=-1.37$ and $N_{\mathrm{H}}=1.3 \times 10^{21} \mathrm{~cm}^{-2}$, and the other with $\log (\xi)=+1.92$ and $N_{\mathrm{H}}=6 \times 10^{21} \mathrm{~cm}^{-2}$.

Our best-fit value for $R, 0.39 \pm 0.05$, is similar to that obtained by Bianchi et al. (2004) for joint fits to a simultaneous XMM-Newton + BeppoSAX observation in 2001 January $(R=0.3-0.5)$ and slightly lower than those obtained by Markowitz, Reeves \& Braito (2006) for an $X M M-N e w t o n$ long-look $(R=0.51 \pm 0.04)$ and Perola et al. (2002) for two BeppoSAX observations in $1998(R=0.6-0.7)$.

Bianchi et al. (2004) reported a high-energy rollover at 130-170 keV (best-fit values depend on the model used; uncertainties were $\sim 10-20$ $\mathrm{keV}$ ) which we do not confirm; our lower limit is $330 \mathrm{keV}$, consistent with rollovers reported by Perola et al. (2002).

Our best-fit value for power-law photon index $\Gamma, 1.88 \pm 0.02$, is very close to values reported in the aforementioned papers.

$$
\text { NGC } 3783
$$

RXTE spectral data have been previously published by Markowitz et al. (2003a), though they only included data up until 2002 April and did not use HEXTE data.

NGC 3783's complex warm absorber properties have been studied extensively with gratings observations (e.g., Netzer et al. 2003, McKernan et al. 2007). Three zones of warm absorption introduce significant continuum curvature in the spectrum above 2 keV. Following Reeves et al. (2004), we included absorbers with $N_{\mathrm{H}}=1.1 \times 10^{21}, 1.2 \times 10^{22}$, and $4.4 \times 10^{22} \mathrm{~cm}^{-2}$ and $\log (\xi)=-0.1,2.1$, and 3.0, respectively.

${ }^{5} \xi \equiv L_{\text {ion }} n_{\mathrm{e}}^{-1} r^{-2}$, where $L_{\text {ion }}$ is usually defined as the isotropic 1-1000 Ryd ionizing continuum luminosity, $n_{\mathrm{e}}$ is the electron number density, and $r$ is the distance from the central continuum source to the absorbing gas. $\xi$ has units of $\mathrm{erg} \mathrm{cm} \mathrm{s}^{-1}$. 
We clearly detected the Compton hump with a strength $R=0.41 \pm 0.08$, in close agreement with Markowitz et al. (2003a) as well as with Perola et al. (2002) using data from the BeppoSAX observation in 1998 June. Using the same BeppoSAX data but different warm absorber model codes and broadband models, Perola et al. (2002) and De Rosa et al. (2002) reported high-energy rollovers at $156_{-40}^{+37}$ and $340_{-107}^{+560} \mathrm{keV}$, respectively; however, we find no evidence for a high-energy rollover $\left(E_{\text {roll }}>350 \mathrm{keV}\right)$.

\section{NGC 5548}

RXTE spectral data have been previously published by Markowitz et al. (2003a), though they only included data up until 2003 January and did not use HEXTE data.

We modeled three zones of warm absorption in this source based on Steenbrugge et al. (2005a), with $N_{\mathrm{H}}=2.5 \times 10^{21}, 1.0 \times 10^{21}$, and $6 \times 10^{20}$ $\mathrm{cm}^{-2}$, and $\log (\xi)=+2.3,+1.9$, and -0.2 , respectively.

Our best-fit value of $R, 0.13 \pm 0.04$, is somewhat smaller than values reported previously, including Liu et al. (2010) for a series of Suzaku monitoring observations obtained in $2007\left(R=0.79_{-0.32}^{+0.35}\right.$ for the summed spectrum), Perola et al. (2002) for a BeppoSAX observation in 1997 $\left(R=0.54_{-0.13}^{+0.20}\right)$, Bianchi et al. (2004) for joint fits to a simultaneous BeppoSAX $+X M M-N e w t o n$ spectrum in $2001\left(R=0.45_{-0.16}^{+0.20}\right)$, and Markowitz et al. (2003a)for $R X T E$-PCA data covering 1999-2003 $\left(R=0.41_{-0.08}^{+0.02}\right)$. Best-fit values of $\Gamma$ in those papers are in the range $1.59-1.74$. It is interesting to note that the 2007 Suzaku campaign caught the source at a relatively low flux level, with a value of $F_{2-10}$ for the summed spectrum of $1.9 \times 10^{-11} \mathrm{erg} \mathrm{cm}^{-2} \mathrm{~s}^{-1}$, a factor of three lower than our best-fit $2-10 \mathrm{keV}$ flux, $6.0 \times 10^{-11} \mathrm{erg} \mathrm{cm}^{-2} \mathrm{~s}^{-1}$. As our data cover the period from 1996 to 2007, this is consistent with the notion that the absolute normalization of the Compton reflection component may have remained constant while the 2-10 keV nuclear flux had dropped by a factor of $\sim 3$ during the Suzaku campaign in 2007 June-August, i.e., the observed reflected flux had not yet responded to the drop in continuum flux by 2007. One possibility is that the bulk of the Compton-reflecting material is located at least several light years from the X-ray nuclear continuum source.

We do not confirm previous reports of high-energy rollovers using BeppoSAX (Bianchi et al. 2004, Perola et al. 2002) or Suzaku (Liu et al. 2010 , who report $E_{\text {roll }}=75_{-15}^{+85} \mathrm{keV}$ ), finding a lower limit to $E_{\text {roll }}$ of $260 \mathrm{keV}$.

\section{Mkn 509}

Previous results on the warm absorbers for this object (McKernan et al. 2007, Smith et al. 2007) indicate a negligible impact on the PCA spectrum. The Compton reflection component is significantly detected but is weak. Our best-fit value of $R, 0.15 \pm 0.05$ is somewhat lower than values reported by De Rosa et al. (2004) for a variety of model fits to BeppoSAX spectra obtained in 1998 and $2000(R \sim 0.8-1.1)$ or Perola et al. (2002) for the 1998 observation $\left(0.58_{-0.30}^{+0.39}\right)$. These works also reported high-energy rollovers with best-fit values in the range $67-115 \mathrm{keV}$; we do not find any evidence for a rollover, with $E_{\text {roll }}>220 \mathrm{keV}$.

\section{MR 2251-178}

Based on previous studies of this object's warm absorbers (e.g, Kaspi et al. 2004), there is negligible impact on the PCA spectrum. We do not find evidence for a Compton reflection hump, broadly consistent with very low values of $R$ modeled by Orr et al. (2001; best-fit values of $R \sim 0.2-0.4$ ) using spectra obtained from a pair of BeppoSAX observations in 1998.

We find only marginal evidence for a rollover at $103_{-30}^{+40} \mathrm{keV}: \chi^{2}$ falls by 10.1 for 1 less dof while the photon index steepens by 0.07 (from $1.63 \pm 0.02$ to $1.56 \pm 0.03)$. This rollover energy is consistent with that claimed by Orr et al (2001: best-fit values near $102-133 \mathrm{keV}$ depending on the model used). However, in our spectrum, much of this improvement in fit is likely associated with trying to fit a small artificially narrow "dip" at $100 \mathrm{keV}$ associated with HEXTE background subtraction. Ignoring the bins around this energy, adding a rollover to the model causes $\chi^{2}$ to drop by only $\sim 6$ for 1 less dof (significant at the $\sim 96 \%$ confidence level according to an $F$-test). Furthermore, there is not much improvement visually in the data/model residuals above $\sim 50 \mathrm{keV}$. We caution that it is unclear from our spectral modeling whether or not the rollover is a real feature.

\section{NGC 3516}

Markowitz et al. (2003a) have published RXTE-PCA spectral data taken up through 2000 February. We include HEXTE data, as well as additional data taken in 2000-2002 and in 2005, but this additional data represents an increase in good PCA exposure time of only $27 \%$ compared to Markowitz et al. (2003a).

This source is known to have several zones of ionized absorption along the line of sight (see Turner et al. 2008 and references therein), and the cold and ionized absorbers can display variability in column depth on both rapid (tens of ks) and long (several years) time scales. The absorber with the most impact on modeling of the long-term average PCA spectrum is the so-called "heavy" component, or "Zone 3," described in Turner et al. (2008). We modeled this component with a column density fixed at $2.0 \times 10^{23} \mathrm{~cm}^{-2}, \log (\xi)$ fixed at 2.19 , and covering fraction left as a free parameter but expected to be near $50 \%$ (we find $56 \%$ in our best-fit model). Other warm absorber components discussed in Turner et al. (2008) such as the "UV absorber" or more highly-ionized absorbers have negligible impact on the PCA spectrum and are ignored.

Our best-fit values of $\Gamma(1.82 \pm 0.04)$ and $R(0.31 \pm 0.09)$ for the reflection model are slightly lower than the best-fit values derived by Markowitz et al. (2008) for the $0.3-76 \mathrm{keV}$ Suzaku spectrum $\left(\Gamma=1.97_{-0.03}^{+0.01}\right.$ and $R=1.8_{-0.5}^{+0.4}$ for their "PC2" model). The discrepancy may be at least partially attributed to degeneracies between $\Gamma$ and $R$; forcing $\Gamma$ to be 1.97 in our fits, $R$ rises to $\sim 0.9$. Much of the remainder of the discrepancy may be attributed to intrinsic source spectral variability. Specifically, it is interesting to note that the observed 2-10 keV and 12-76 keV fluxes for the RXTE spectrum are $4.4 \times 10^{-11}$ (PCA) and $9.3 \times 10^{-11} \mathrm{erg} \mathrm{cm}^{-2} \mathrm{~s}^{-1}$ (average of HEXTE A and B responses), respectively, while for the Suzaku observation $F_{2-10}$ and $F_{12-76}$ were $2.3 \times 10^{-11}$ and $11 \times 10^{-11} \mathrm{erg} \mathrm{cm}^{-2} \mathrm{~s}^{-1}$, respectively. That is, the ratio $F_{12-76} / F_{2-10}$ was twice as high during the Suzaku observation, indicating a large degree of spectral variability, and requiring a larger relative normalization for the Compton reflection component in the Suzaku observation.

Markowitz et al. (2003a) found lower a value for $\Gamma(\sim 1.6)$, but this discrepancy is likely due to the fact that those authors did not include the partial-covering, "heavy" warm absorber (described by Turner et al. 2005) in their modeling, affecting determination of the broadband continuum and thus (the mildly-degenerate) photon index and Compton reflection strength. In fact, removing the partial-covering absorber from our fits, we obtain a fit with roughly similar values of $\Gamma$ and $R(\sim 0.3)$ to Markowitz, Edelson, \& Vaughan $(2003)$, but the fit quality is poor $\left(\chi_{r}^{2} \sim 3.1\right)$. Similarly, Bianchi et al. (2004), modeling simultaneous XMM-Newton and BeppoSAX observations conducted in 2001, include a full-covering warm absorber with $N_{\mathrm{H}} \sim 1.5 \times 10^{22} \mathrm{~cm}^{-2}$ in their model, but they do not model the partial-covering "heavy" component, yielding what may be a spuriously low value of $\Gamma(\sim 1.5)$.

Finally, we note that including a high-energy rollover offers insignificant improvement to our fit, with $E_{\text {roll }}>220 \mathrm{keV}$. 
Data taken from 2000 November to 2001 May (modified Julian day (MJD) 51,850-52,050), affected by the passage of the compact cloud cross the line of sight (Lamer et al. 2003), were ignored during spectral fitting.

Two zones of warm absorption, with $N_{\mathrm{H}}=1 \times 10^{21}$ and $2 \times 10^{21} \mathrm{~cm}^{-2}$ and $\log (\xi)=1.2$ and 2.9 , respectively, were included in the spectral fits following the XMM-Newton-RGS analysis of Markowitz et al. (2009). We also included a full-covering zone of cold absorption with $N_{\mathrm{H}}$ as a free parameter, obtaining $1.5 \pm 0.9 \times 10^{21} \mathrm{~cm}^{-2}$ in the best-fit model.

Markowitz et al. (2009) also presented joint spectral fits to PCA + HEXTE data up to $100 \mathrm{keV}$, plus joint fits with four-channel Swift-BAT data. They reported a rollover at $90 \pm 20 \mathrm{keV}$, driven primarily by the inclusion of the BAT spectrum; we find no significant evidence for a rollover in the current spectrum $\left(E_{\text {roll }}>210 \mathrm{keV}\right)$.

The relatively low value of $\Gamma$ we obtain, $1.69 \pm 0.04$, is consistent with previous results from XMM-Newton and RXTE spectral fits (Cappi et al. 2006).

\section{$N G C 4593$}

We included one warm absorber zone, with $N_{\mathrm{H}}=3 \times 10^{21} \mathrm{~cm}^{-2}$ and $\log (\xi)=2.4$ (Markowitz \& Reeves 2009; McKernan et al. 2003).

Using Suzaku data, Markowitz \& Reeves (2009) modeled a Compton reflection hump with a strength $R=1.08 \pm 0.20$, however that particular observation occurred in 2007 December during a low-flux state: $F_{2-10}$ was a factor of $\sim 3.5$ lower than the historical long-term average (Figure 2). The fact that we measure an $R$ value of $0.34 \pm 0.09$ for the long-term spectrum is consistent with the notion of a Compton reflection component that is constant in absolute normalization (i.e., it did not respond to the drop in continuum flux during the Suzaku observation). This would require that the bulk of the reflecting material is located a minimum of $\sim$ a lt-yr from the central X-ray source, as the $2-10 \mathrm{keV}$ flux light curve (Figure 2) indicates a decline from average 2-10 keV flux levels before MJD $\sim 54100$ to the lower flux level during the Suzaku observation on MJD MJD 54449.

Our value of $R$ is only barely inconsistent with that obtained by Guainazzi et al. (1999) from the BeppoSAX observation in 1997 ( $R \sim 1.0$ with typical uncertainties of $\sim 0.5)$, which caught the source at a typical flux level $\left(F_{2-10} \sim 4 \times 10^{-11} \mathrm{erg} \mathrm{cm}^{-2} \mathrm{~s}^{-1}\right)$. We find a photon index of $1.85 \pm 0.03$, consistent with that reported by Brenneman et al. (2007) for an XMM-Newton long-look and Guainazzi et al. (1999).

There is no evidence for a rollover in this source based on our $R X T E$ spectrum $\left(E_{\text {roll }}>335 \mathrm{keV}\right)$ or based on the BeppoSAX observation $\left(E_{\mathrm{roll}}>\right.$ $222 \mathrm{keV}$ and $\gtrsim 150 \mathrm{keV}$ from Perola et al. 2002 and Guainazzi et al. 1999, respectively).

\section{NGC 7469}

The warm absorbers (measured by e.g., Blustin et al. 2007) do not significantly impact the modeling of the PCA spectrum and were ignored in our modeling. Previous RXTE spectral results were published by Nandra et al. (2000) and Markowitz et al. (2003a) for the 1996 intensive monitoring campaign; our analysis also includes data from the longer-term monitoring campaign, from 2003 April to 2009 July.

Our best-fit value of $R, 0.7 \pm 0.2$ is broadly consistent with values reported by Markowitz et al. (2003a) and Perola et al. (2002, for the 1999 BeppoSAX observation), and slightly lower than values reported by De Rosa et al. (2002) for the BeppoSAX observation for a range of spectral models (best-fit values in the range 0.9-1.8 with uncertainties as high as 0.6).

Using BeppoSAX data, De Rosa et al. (2002) and Perola et al. (2002) each reported a high-energy rollover; depending on the spectral model tested, best-fit values range from $\sim 140$ to $\sim 260 \mathrm{keV}$ with very large uncertainties, but we do not find evidence for a rollover in our data $\left(E_{\text {roll }}>\right.$ $220 \mathrm{keV})$.

\section{$3 C 111$}

RXTE observations have been concentrated into five short campaigns over 1997 to 2003, plus continuous monitoring since 2004 March. The long-term PCA light curve shows long-term variations by a factor of $\sim 3-4$ (Figure 2).

RXTE PCA+HEXTE spectra were previously published by Eracleous, Sambruna \& Mushotzky (2000); the good time exposures for PCA and HEXTE were 33 and $13 \mathrm{ks}$, respectively. Lewis et al. (2005) published an RXTE observation simultaneous with an XMM-Newton long-look in 2001 March; good exposure times were $57 \mathrm{ks}$ for the PCA and $18 \mathrm{ks}$ for each HEXTE cluster.

The total X-ray absorbing column along the line of sight measured from various X-ray missions from $H E A O-1$ to ASCA all show evidence for absorption by a column of cold gas in excess of the Galactic column inferred from 21-cm measurements (Reynolds et al. 1998). This is likely due to a molecular cloud lying along the line of sight to 3C 111 (Bania et al. 1991); the cloud's molecular gas (H II and metals/dust) will contribute to the total X-ray absorption but not to the measured $21 \mathrm{~cm}$ radio emission. We herein adopt a molecular hydrogen column density of $9 \times 10^{21}$ $\mathrm{cm}^{-2}$ (Bania et al. 1991, estimated from CO emission measurements) for a total Galactic nonionized hydrogen column of $1.2 \times 10^{22} \mathrm{~cm}^{-2}$ in all fits. Warm absorption does not significantly affect the PCA spectrum.

Previous results on BLRGs, including Wozniak et al. (1998) and Eracleous et al. (2000), indicated that BLRGs tend to have Compton reflection humps and $\mathrm{Fe} \mathrm{K} \alpha$ lines which are generally weak compared to those of normal Seyferts. We find no evidence for a Compton reflection hump in our spectrum, generally consistent with previous results from RXTE, BeppoSAX, and Ginga observations, where upper limits to $R$ were reported or best-fit values of $R$ were $\lesssim 0.5$ (above-mentioned references; Grandi et al. 2006).

We also find no evidence for a rollover, with $E_{\text {roll }}>230 \mathrm{keV}$; a rollover was claimed by Grandi et al. (2006) for a BeppoSAX observation in $1998\left(146_{-68}^{+224} \mathrm{keV}\right)$.

\section{$3 C 120$}

No warm absorbers significantly affect the spectrum above $2 \mathrm{keV}$. Joint fits to PCA + HEXTE spectra have been published previously by Gliozzi et al. (2003); their spectral data, obtained in 1997, contained 100 (40) ks of good exposure time for the PCA (each HEXTE cluster) and only included data up to $50 \mathrm{keV}$ in HEXTE. Eracleous et al. (2000) published joint fits to the PCA + HEXTE spectrum (up to $100 \mathrm{keV}$ ) obtained from a 2-day RXTE observation in 1998. Our best-fit results for the Compton reflection strength and our lack of evidence for a rollover for our spectrum is consistent with results from those papers. Our results are also roughly consistent with best-fit values for the Compton reflection strength obtained by Kataoka et al. (2007; Suzaku) and Zdziarski \& Grandi (2001, BeppoSAX, and also BeppoSAX + CGRO-OSSE joint fitting), $\sim 0.4-0.6$ with uncertainties typically $\sim 0.2-0.4$.

Modeling a rollover causes $\chi^{2}$ to drop by only 3.2 for 1 less dof between the reflection and rollover models, with large changes to both $\Gamma$ (flattens by 0.2 to $\sim 1.6$ ) and $R$ (goes to 0 ), and also yields a large uncertainty in $E_{\text {roll }}, 190_{-70}^{+310} \mathrm{keV}$. While this value of $E_{\text {roll }}$ is consistent with those reported by Zdziarski \& Grandi (2001), Grandi et al. (2006), and Wozniak et al. (1998, from joint ASCA+OSSE fits), the improvement in fit is not significant and the best-fit values for $\Gamma$ and $R$ are inconsistent with previous results (e.g., Grandi et al. 2006; Gliozzi et al. 2003; and Zdziarski \& Grandi 2001 each find best-fit values of $\Gamma$ closer to 1.8), and we thus conclude that the reflection model is the best description of the data with a conservative estimate for the lower limit on $E_{\text {roll }}$ of $120 \mathrm{keV}$. 
RXTE-PCA spectral data obtained from observations occurring from 1996 through early 1999 were published by Lamer et al. (2000).

The evidence for a rollover in our spectrum is not strong: $\chi^{2}$ falls by only 4 for 1 less dof, and data/model residuals do not improve noticeably. Moreover, $\Gamma$ flattens by 0.1 , to 1.80 , and the column density of the Compton-thin absorber $N_{\mathrm{H}}$ falls to $<0.9 \times 10^{22} \mathrm{~cm}^{-2}$. However, most previous studies by various X-ray missions (Bianchi et al 2003, 2004; Risaliti 2002) indicate that $\Gamma$ is closer to 1.9-2.0 (more consistent with this source being an "obscured Narrow Line Seyfert 1", e.g., Dewangan \& Griffiths 2005), and $N_{\mathrm{H}} \sim(2-4) \times 10^{22} \mathrm{~cm}^{-2}$, similar to our results when a rollover is not included in the model. We thus conclude that the reflection model is the best description of the spectrum.

Most values of $R$ reported in the literature (above references, also Perola et al. 2002) are similar to ours, with best-fit values of $R$ in the range 1.0-1.5. We do not confirm the rollovers of $110-140 \mathrm{keV}( \pm \sim 30 \mathrm{keV}$ depending on the model) claimed by Bianchi et al. (2004) for a simultaneous BeppoSAX/XMM-Newton observation in 2001 February; we obtain a lower limit of $209 \mathrm{keV}$.

\section{$M C G-5-23-16$}

RXTE observations have been concentrated in several campaigns instead of sustained monitoring, but the average $2-10 \mathrm{keV}$ flux within each campaign never deviates by more than $\sim 25 \%$ from the mean $2-10 \mathrm{keV}$ flux, $9.6 \times 10^{-11} \mathrm{erg} \mathrm{cm}^{-2} \mathrm{~s}^{-1}$. Our best-fit model includes a single, full-covering cold absorber with $N_{\mathrm{H}}=3.5 \pm 0.5 \times 10^{22} \mathrm{~cm}^{-2}$; this is only about a factor of two higher than the column modeled by Reeves et al. (2007) using Suzaku and Braito et al. (2007) using XMM-Newton and Chandra-HETGS.

Weaver et al. (1998) published the RXTE PCA spectrum from the 1996 November campaign ( $80 \mathrm{ks}$ of good exposure time). For an inclination angle of $50^{\circ}$, best-fit values of $R$ are $0.36_{-0.22}^{+0.24}$ for $E_{\text {roll }}$ fixed at $500 \mathrm{keV}$ (consistent with our results), and $0.45 \pm 0.23$ for $E_{\text {roll }}$ fixed at $200 \mathrm{keV}$. However, Reeves et al. (2007) found a total Compton reflection strength of $1.1 \pm 0.2$ (assuming an inclination angle of $45^{\circ}$ ) using Suzaku, a factor of $\sim 2.5$ higher than our best-fit value. The $2-10$ and $15-100 \mathrm{keV}$ fluxes reported by Reeves et al. (2007) are virtually identical to what we measure, $F_{2-10}=9.6 \times 10^{-11} \mathrm{erg} \mathrm{cm}^{-2} \mathrm{~s}^{-1}$ and $F_{15-100}=1.9 \times 10^{-11} \mathrm{erg} \mathrm{cm}^{-2} \mathrm{~s}^{-1}$. The discrepancy may be at least partially attributed to differences in spectral modeling and degeneracies between parameters, particularly $N_{\mathrm{H}}, \Gamma$, and $R$; specifically, we find a value for $\Gamma 0.1$ lower than Reeves et al. (2007; forcing $\Gamma$ to be 1.95 while ignoring data $<5 \mathrm{keV}$ which influence the value of $N_{\mathrm{H}}, R$ rises to $\sim 0.75$ ). The value of $R$ found by Perola et al. (2002) is in agreement with Reeves et al. (2007), given the inclination angles assumed by both papers.

Our lower limit to $E_{\text {roll }}, 170 \mathrm{keV}$, is identical to that found by Reeves et al. (2007). It is consistent with the rollover claimed by Perola et al. (2002) using BeppoSAX, $147_{-40}^{+70} \mathrm{keV}$, and consistent with the results of Zdziarski et al. (1996), who noted that spectral fits to non-simultaneous Ginga + OSSE data suggested a rollover near $200 \mathrm{keV}$.

\section{NGC 4507}

RXTE observations are clustered into two campaigns, one in 1996 which accounts for $94 \%$ of the total good exposure time, and one in 2003. There were three observations with BeppoSAX, in 1997, 1998 and 1999, analyzed by Risaliti (2002). As reported in their best-fit model with reflection (their "Model C"), from 1997 to 1999 , the $2-10 \mathrm{keV}$ flux dropped by a factor of 2.1 from $1.84 \times 10^{-11}$ to $0.87 \times 10^{-11}$ erg $\mathrm{cm}^{-2} \mathrm{~s}^{-1}$, while $R$ increased from $0.7 \pm 0.2$ to $2.0 \pm 0.5$, consistent with the Compton hump absolute normalization staying constant despite the drop in power-law normalization. However, from our modeling, our best-fit value of $R$ is only $0.4 \pm 0.1$ despite our best-fit $2-10 \mathrm{keV}$ flux, $1.43 \times 10^{-11}$ erg cm $\mathrm{cm}^{-2} \mathrm{~s}^{-1}$, lying in that same range. Best-fit values of $\Gamma$ and $N_{\mathrm{H}}$ do not differ by more than $\sim 0.2$ and a factor of 1.5 , respectively, between the $R X T E$ and BeppoSAX models, and so the cause of the discrepancy is not obvious. One possibility, however, is that the high reflection component normalization value could indicate a response to a higher past illuminating flux. We do not find significant evidence for a high-energy rollover, with $E_{\text {roll }}>170 \mathrm{keV}$ (consistent with Risaliti 2002).

\section{$\operatorname{Cen} A$}

A comprehensive spectral analysis of all RXTE campaigns through 2009 is given by Rothschild et al. (in prep.). Our best-fit model parameters are in agreement with results in that paper (see also Rothschild et al. 2006 for results on RXTE and INTEGRAL observations up through 2003). Markowitz et al. (2007) present analysis of the Suzaku observation of Cen A in 2005, covering 0.3 to $220 \mathrm{keV}$. They present evidence for a secondary continuum emission component above $4 \mathrm{keV}$, absorbed by a cold column with $N_{\mathrm{H}}=7.0 \times 10^{23} \mathrm{~cm}^{-2}$, though this component is not detected in the RXTE analyses. Neither the current nor any of the above analyses find evidence for a Compton reflection component; previous upper limits to $R$ have usually been close to 0.05 (see also Benlloch et al. 2001). A high-energy rollover is also not required by the $R X T E$ data; we find $E_{\text {roll }}>490 \mathrm{keV}$, similar to the lower limit of $400 \mathrm{keV}$ found by Markowitz et al. (2007). Using CGRO spectra, Kinzer et al. (1995) modeled a rollover at 300- 700 keV (depending on flux level), while Steinle et al. (1998) claimed a break near $150 \mathrm{keV}$.

\section{NGC 4945}

As pointed out by Done et al. (1996), CGRO-OSSE demonstrated that this heavily-obscured source is one of the brightest AGN in the $>100$ keV sky. PCA+HEXTE spectral fits were published previously by Madejski et al. (2000), though they only used data from the fall 1997 campaign.

We excluded data from ObsID 92118-01-40-00, observed on 2006 May 20, due to the presence of spectrally-steep emission below 12 keV which was a factor of $\sim 3$ times the emission for each of all other observations of NGC 4945. The nature and origin of this emission is discussed in Appendix C.

Our spectral fit results are broadly consistent qualitatively with the results of Itoh et al. (2008) for the Suzaku observation. In our model, the socalled "direct" or "transmitted" component is the Compton-thick-absorbed power-law which dominates above $\sim 12 \mathrm{keV}$. A weak, cold Compton reflection component dominates from $\sim 11 \mathrm{keV}$ down to $\sim 6 \mathrm{keV}$. Emission below $5 \mathrm{keV}$ may be thermal emission from a plasma and/or scattered emission (e.g., Itoh et al. 2008); we model this emission as a simple soft power law, but the power-law parameters are very poorly constrained because we have no information below $3 \mathrm{keV}$ and, given the PCA energy resolution, there is likely blending with the Fe line parameters.

An additional contributor to the $<5 \mathrm{keV}$ emission modeled here as a simple power-law is the ultra-luminous transient X-ray (ULX) source Suzaku J1305-4931, located a few arcmin to the SW of the nucleus of NGC 4945, and discovered in a Suzaku observation of NGC 4945 obtained in 2006 January (Isobe et al. 2008). The ULX was not detected in a Suzaku observation of NGC 4945 in 2005 August, nor has it been reported elsewhere, and so the details of the ULX's flux activity as a function of time during early 2006 are not known. However, that source's $0.5-10$ keV flux was only $\sim 2 \times 10^{-12} \mathrm{erg} \mathrm{cm}^{-2} \mathrm{~s}^{-1}$, and the impact on the summed $R X T E$ spectrum is small, affecting only the power-law component below $\sim 10 \mathrm{keV}$.

When we include a high-energy rollover in the model, (the data/model residuals in Figure 30 are particularly compelling in this case) the strength of the Compton reflection hump dwindles from $R=0.07$ to an upper limit of $R<0.03$ as the curving introduced into the continuum by the high-energy rollover accounts for a portion of the already-weak (relative to the absorbed power-law) Compton hump. As a result of 
degeneracy between $E_{\text {roll }}$ and $\Gamma$ and the fact that $\Gamma$ is already poorly constrained due to the strong absorption, the best-fit value of $\Gamma$ becomes rather low, $1.0 \pm 0.1$, when a rollover is modeled.

Itoh et al. (2008) and Guainazzi et al. (2000) each reported a high-energy rollover at $190_{-50}^{+150}$ and $190_{-90}^{+100} \mathrm{keV}$, respectively; the latter is only barely inconsistent at the $90 \%$ confidence level with the rollover we model. Done et al. (1996) modeled the 0.6-400 keV spectrum constructed from non-simultaneous OSSE, Ginga and ASCA data; no strong evidence for a rollover was found.

\section{Circinus}

The good exposure time for this object is dominated by the sampling obtained in 1998.

Matt et al. (1999), who analyzed the spectrum obtained from the BeppoSAX observation in 1998, were the first to extend the spectrum of this source out to $>10 \mathrm{keV}$ and were the first to establish the presence of the absorbed power-law component. With the exception of modeling soft X-ray emission lines, our best-fit model is similar to that of Matt et al. (1999): a Compton-thick-absorbed power-law, a Compton reflection component, a softer power-law component, and an Fe emission line.

In the case of a model with no rollover, the photon index for the soft power law is identical to that of the base power-law, consistent with either scattered or leaked emission. For the model that includes the rollover component both photon indices are poorly constrained and subject to degeneracies. In the context of scattered emission, we find that the optical depth of the scattering medium is $4 \%$ or $47 \%$ when Compton reflection is included in the model and a high-energy rollover is excluded or included, respectively (alternately, partial covering fractions of $96 \%$ or $53 \%$ if the soft emission is "leaked"). In our best-fit model, we find roughly similar column densities for the Compton-thick absorber and consistent $R$ values as Matt et al. (1999).

We confirm the presence of the Compton-thick absorbed power-law component, e.g., the "directly transmitted" nuclear emission. A model without that component, and wherein the peak of the spectrum at $\sim 20 \mathrm{keV}$ is explained by a dominating Compton reflection component, yields a poor fit $\left(\chi^{2} /\right.$ dof $\left.=1473 / 63\right)$. Allowing for the Compton reflection component to undergo Compton-thick absorption yields an unsatisfactory fit with $\chi^{2} / \mathrm{dof}=216 / 64$ and with poor residuals, and so this type of model is not discussed further.

Of all the objects in our sample, the evidence for a high energy rollover is the strongest in this object, with $\chi^{2}$ dropping by $>50$ for 1 less dof. However, given that the hard X-ray power-law undergoes Compton-thick absorption and suffers a rollover below $\sim 15 \mathrm{keV}$, there is degeneracy between the best-fit values $\Gamma$ and $E_{\text {roll }}$, as shown in Figure 22 In this case we did not allow $E_{\text {roll }}$ to drop below a hard limit of $40 \mathrm{keV}$ in order to minimize the impact on modeling of the Compton reflection component peaking at 20-30 keV.

Sambruna et al. (2001) published the 6-30 keV PCA spectrum obtained during the observations in 2000, which were simultaneous to the Chandra-HETGS observation, the focus of their paper. They also modeled a Compton reflection component plus absorbed power-law, in addition to neutral and ionized Fe $\mathrm{K}$ lines, though constraints on $\Gamma$ were very poor and the strength of the Compton reflection component was not reported.

\section{NGC 7582}

In the course of our modeling, we found two models which yielded approximately equally good statistical descriptions of the data. In the first model, "REFL", a single power-law component with $\Gamma=1.79 \pm 0.10$ is absorbed by a full-covering column of Compton-thin gas with $N_{\mathrm{H}}=1.4 \pm 3 \times 10^{23} \mathrm{~cm}^{-2}$, and is accompanied by a strong Compton reflection component, $R=3.3_{-0.9}^{+1.6} ; \chi^{2} / \mathrm{dof}=36.3 / 33$. Using the PEXRAV model, values of $R$ greater than two do not necessarily have a direct geometrical meaning but may indicate a geometry more complex than a simple reflecting slab or may indicate anisotropic beaming of the primary continuum.

The second model, "PC", includes a partial-covering Compton-thick absorber $\left(f_{\text {cov }}=60 \% ; N_{\mathrm{H}}=3.6 \pm 0.6 \times 10^{24} \mathrm{~cm}^{-2}\right)$ in addition to the full-covering Compton-thin absorber $\left(N_{\mathrm{H}}=2.4 \pm 0.4 \times 10^{23} \mathrm{~cm}^{-2}\right) ; \chi^{2} /$ dof was $40.0 / 32$. Adding a Compton reflection hump to the PC model did not yield any improvement in fit, as there is complete degeneracy between the strength of the Compton reflection hump and the Compton-thick absorbed power-law, both of which peak near 20-30 keV; this held whether or not the reflection component was modeled to be obscured by the same Compton-thick gas obscuring the power-law component $\left(\Gamma=1.84 \pm 0.12\right.$; power-law normalization $A=1.8_{-0.6}^{+1.0} \times 10^{-2} \mathrm{ph} \mathrm{keV}^{-1} \mathrm{~cm}^{-2} \mathrm{~s}^{-1}$ at $1 \mathrm{keV})$.

In their modeling of a spectrum of NGC 7582 obtained with BeppoSAX in 1998, Turner et al. (2000) also explored models of these two types. They favored a PC type model over a REFL type model on physical grounds: in the latter, they found the Compton hump strength $R$ to be near 6 (assuming an inclination $\cos i=0.45$ ), which was incompatible with the small value of the EW for the Fe K $\alpha$ line (assuming that a Compton hump with a value of $R$ near 1 will be associated with an Fe line with an EW near $150 \mathrm{keV}$, George \& Fabian 1991). In our REFL model, we find an Fe line EW of $331 \pm 94 \mathrm{eV}$, which is not incompatible with the observed Compton hump strength, and so we cannot reject this model on this basis alone (see Figure 31). However, Bianchi et al. (2007) analyzed Chandra and Hubble Space Telescope images and concluded that there exists a Compton-thick torus plus large-scale Compton-thin material, in agreement with the complex absorption structure first quantified by Turner et al. (2000).

A model more complex than either PC or REFL may in fact be preferred. In the context of the PC model, we might reasonably expect that if there is Compton-thick absorption along the line of sight, there may likely be reflected emission originating in circumnuclear Compton-thick gas. Bianchi et al. (2009), in their modeling of a series of four Suzaku spectra of NGC 7582 obtained in 2007, include both a partial-covering Compton-thick absorber and a Compton reflection component. In the PC model as applied to the current RXTE spectrum, we are simply not sensitive to the presence of a Compton reflection component; we cannot rule out the presence of a Compton reflection component with a strength similar to that modeled by Bianchi et al. (2009).

For the REFL model, we found no significant improvement to the fit when a high-energy rollover was modeled, obtaining a lower limit of 200 $\mathrm{keV}$. For the PC model, degeneracy made the addition of a rollover unconstrainable.

\section{$3 C 273$}

3C 273 has been studied with every major X-ray observatory to date. The X-ray spectrum typically resembles those of Seyfert 1s with an Fe line and Compton reflection hump likely visible at during low flux states and likely diluted by an additional X-ray continuum contribution from the jet (e.g., Grandi et al. 1997, Kataoka et al. 2002).

In our fits, adding a Compton reflection hump to a model consisting only of a power-law plus a Gaussian component significantly improves the fit, as $\chi^{2}$ drops by 32 for 1 less dof. However, the strength of the Compton hump is quite low: $R=0.07 \pm 0.03$.

The flat photon index for our power-law component is in general agreement with previous results, such as Kataoka et al. (2002), who presented RXTE-PCA only spectral data from monitoring in 1996-2000, and a set of BeppoSAX observations analyzed by Grandi et al. (2006).

If a jet component does make a significant contribution to the $X$-ray spectrum then a power-law rollover associated with thermal Comptonization at 100-300 keV may be masked by the non-thermal jet emission. We find no significant evidence for a rollover, with $E_{\text {roll }}>410 \mathrm{keV}$. 

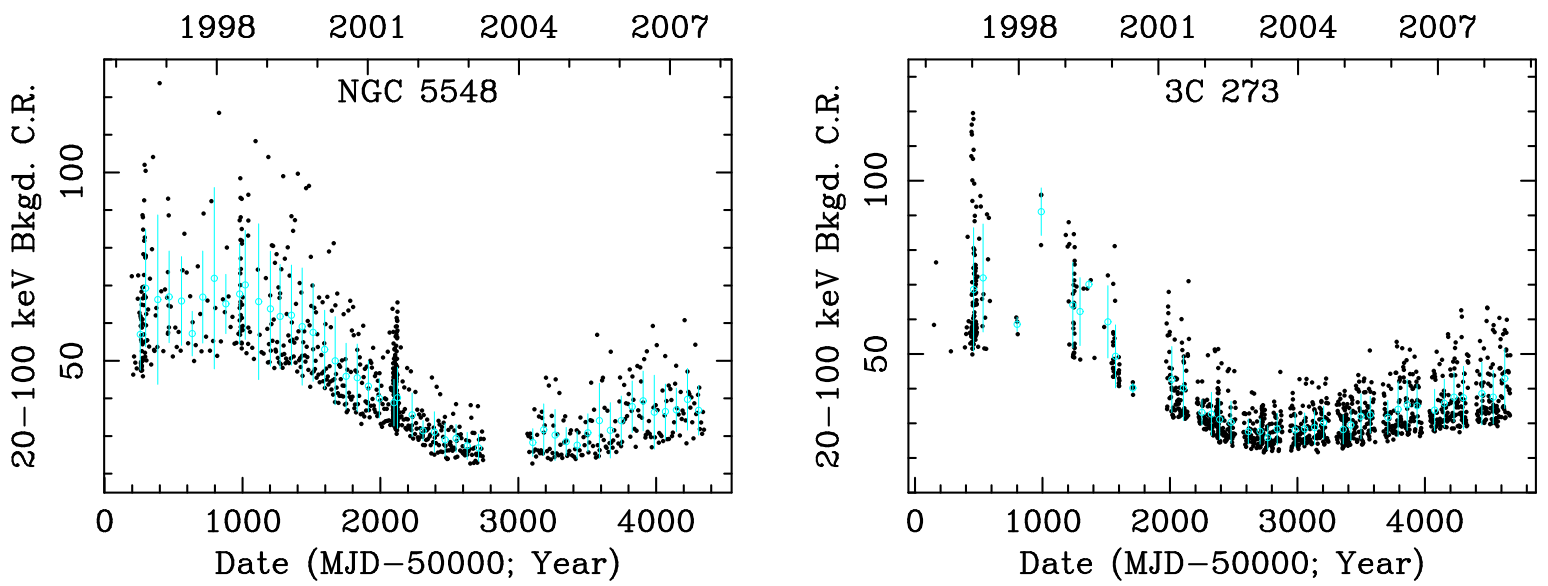

FIG. 32.- Light curves showing 20-100 keV HEXTE cluster B background count rates (after application of screening criteria) for two selected targets, NGC 5548 (left) and 3C 273 (right), illustrating the long-term decreasing trend in the average background flux due to the decrease in altitude, and thus particle flux intercepted by, the RXTE spacecraft. Additional long-term trends, such as the increase after $\sim 2004$, are likely the result of solar activity. Blue points denote the averages over $\sim 80$-day bins, with error bars signifying the standard deviation of count rates within each bin.

\section{$3 C 454.3$}

The RXTE monitoring data used herein were obtained as part of a multiwavelength campaign that occurred when the source went into a bright flaring state in 2005. The PCA light curve (though not spectrum) is presented as a part of a multi-wavelength analysis in Jorstad et al. (2010); detailed discussion on the origin of the high-energy continuum emission can be found in, e.g., that paper, Ghisellini et al. (2007), and Pian et al. (2006). For consistency, we did not include archival RXTE monitoring data that did not use the offset pointing position.

\section{Mkn 421}

Mkn 421 is one of the most extensively studied BL Lac objects across the electromagnetic spectrum. The RXTE data used here were usually obtained as part of multi-wavelength campaigns when the source was in outburst. Despite being a steep spectrum source, Mkn 421 is one of the brightest AGN in X-rays when it flares, yielding high quality spectra with HEXTE. PCA and HEXTE spectral and light curve data during flares, and modeling of the broadband SED, have been published in, e.g., Giebels et al. (2007), Rebillot et al. (2006), and Błazejowski et al. (2005). The source's extreme levels of X-ray flux and spectral variability over a wide range of time scales have been well-documented previously (e.g., Cui 2004), and the average 2-10 keV flux considered here may be a factor of 3-4 less than the flux during the brightest flaring states. We found that a simple power-law fit to the long-term summed PCA + HEXTE spectrum yielded a poor fit, with $\chi^{2} /$ dof $=298 / 81$ and poor data-model residuals. Using a broken power-law to allow for a model representing a slowly bending continuum allowed for a good fit. Our results are similar to those of Giebels et al. (2007) and Foschini et al. (2006) for XMM-Newton data.

\section{IES $1959+650$}

The RXTE data used here were gathered as part of multi-wavelength (typically including X-ray, TeV, optical, and radio) campaigns and have been published previously by Giebels et al. (2002), Krawczynski et al. (2004), and Gutierrez et al. (2006) for the 2000, 2002, and 2003 campaigns, respectively.

Gutierrez et al. (2006) demonstrated that the X-ray spectrum flattens as flux increases, with $\Gamma \sim 2.9$ (1.6) when the X-ray flux is a factor of $\sim 3$ higher (lower) than the long-term average flux, $1.6 \times 10^{-10} \mathrm{erg} \mathrm{cm}^{-2} \mathrm{~s}^{-1}$. Furthermore, the X-ray band lies near the peak of the synchrotron component in the broadband SED. Consequently, our spectral fitting found that a broken power-law model (with $\left.\chi^{2} / \mathrm{dof}=47.4 / 62\right)$ was a better fit than an unbroken power-law ( $\chi^{2} / \mathrm{dof}=62.6 / 64$, with poor residuals below $\sim 8 \mathrm{keV}$ ). Previous to the $R X T E$ programs, BeppoSAX observed this source in 1997; the reader is referred to Beckmann et al. (2002) and Donato et al. (2005) for those spectral fit results.

\section{B. LONG-TERM VARIABILITY IN THE HEXTE BACKGROUND}

In this Appendix, we summarize the long-term variability of the HEXTE background; the reader is referred to Rothschild et al. (1998) and Gruber et al. (1989) for further details of the internal backgrounds of the HEXTE detectors and the NaI/CsI scintillators comprising the Hard $\mathrm{X}$-Ray and Low-Energy Gamma Ray Experiment (A4) aboard HEAO-1, respectively.

The HEXTE background count rate is dominated by internal activation features. The total count rate thus depends strongly on the particle flux intercepted by the spacecraft, as measured by two independent particle monitors, one for each HEXTE cluster. The background flux is dominated by emission lines resulting from short-term activation by geomagnetically trapped protons encountered in the SAA as well as by cosmic rays, and so the instantaneous background rate depends strongly on the location of the spacecraft within its orbit, i.e., it is highest during and immediately after SAA passage and begins to exponentially decay once the spacecraft exits the SAA. Satellite orientation with respect to the geomagnetic field and instantaneous geomagnetic rigidity rollover are also key factors affecting the total background rate. Even after screening out data gathered within tens of minutes of SAA passage, the background count rate is still quite variable on timescales of hours to a day and from one $\sim 1 \mathrm{ks}$ duration observation to the next, depending on the closeness of a particular observation to the previous SAA passage.

The strongest emission feature in the background is an emission feature near $30 \mathrm{keV}$ due to the $\mathrm{K}$ lines from the Te daughters of various I decays. From 58 to $87 \mathrm{keV}$ is a blend of emission lines whose origins include ${ }^{121} \mathrm{I}$ decay at $58 \mathrm{keV}$ and fluorescence of Pb in the collimator by both charged particles and cosmic X-rays (from celestial sources outside the field of view, including the CXB). At $191 \mathrm{keV}$, there is an emission feature due to decay of ${ }^{123} \mathrm{I}$, and there is a weak broad line at $154 \mathrm{keV}$ due to K-escape lines from the $191 \mathrm{keV}$ complex. There is also a flat $\beta$-decay continuum component. In addition, there is a wide range in activation half-lives (see Table 1 in Gruber et al. 1989; other, weaker emission features are discussed therein).

However, there is an additional long-term trend in the total background flux: as the altitude of the RXTE spacecraft has dropped from approximately $590 \mathrm{~km}$ in 1996 to $480 \mathrm{~km}$ in 2007, the spacecraft has been intercepting smaller regions of the SAA and experiencing a relatively stronger geomagnetic field strength. The increased geomagnetic rigidity rollover as a function of altitude makes it more difficult for relatively lower energy particles to get through, so the average particle flux intercepted by the spacecraft has been decreasing as documented by Fürst et al. (2009). Consequently, the average HEXTE background flux has dropped by $\sim 50 \%$ (from $\sim 12$ to $\sim 6 \times 10^{-9} \mathrm{erg} \mathrm{cm}^{-2} \mathrm{~s}^{-1}$ ). Figure A1 illustrates 


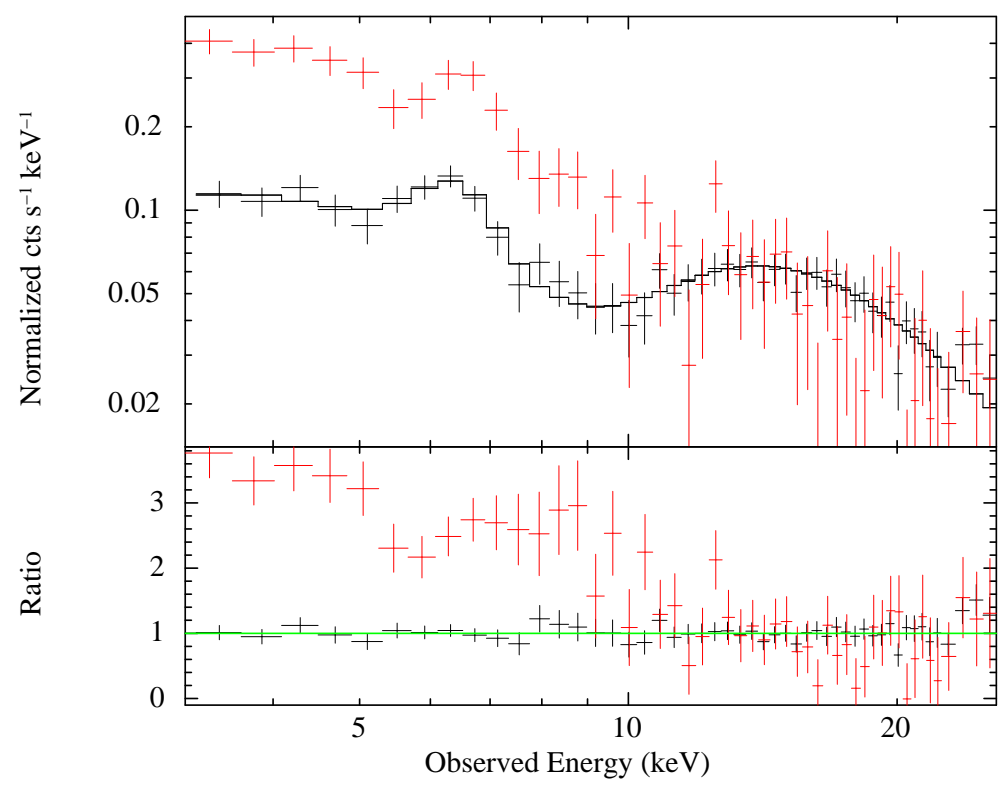

FIG. 33. - 3-26 keV PCA data from the observation of NGC 4945. on 2006 May 20 are shown in black. Red points show the summed 3-26 keV PCA spectrum from all observations in the range 2006 May 10-30, excluding the observation on May 20, along with the best-fit model. In the former spectrum, below $\sim 12$ keV, there is steep-spectrum emission, along with a possible Fe emission line, in excess of the nuclear activity and diffuse thermal emission associated with NGC 4945.

this long-term trend by plotting the HEXTE cluster B background flux in the $20-100 \mathrm{keV}$ band for each observation (after screening criteria were applied) for two of the more consistently-sampled targets in our sample, NGC 5548 and 3C 273.

\section{A POSSIBLE TRANSIENT SOURCE NEAR NGC 4945}

Data from ObsID 92118-01-40-00, observed on MJD 53875, 2006 May 20, were not used in the summed spectrum for NGC 4945. The 2-10 $\mathrm{keV}$ flux was found to be $2.1 \times 10^{-11} \mathrm{erg} \mathrm{cm}^{-2} \mathrm{~s}^{-1}$, a factor of $2.5-3$ greater than the flux during all other RXTE observations of this source, which lie in the range $(3-7) \times 10^{-12} \mathrm{erg} \mathrm{cm}^{-2} \mathrm{~s}^{-1}$. During this time, RXTE was monitoring the source once every 2 days; no other observations during this time, including those on May 18 and 22, show evidence for an abnormally large flux.

The spectrum for the 2006 May 20 observation (see Figure B1) indicates the presence of an unusually steep and bright component below 12 $\mathrm{keV}$, with a line-like feature near $6 \mathrm{keV}$; the spectrum above $12 \mathrm{keV}$ is roughly consistent with that extracted from all other observations obtained during May 10-30 (see below). The PCA background spectra for each individual observation during this time were examined, but nothing unusual was seen for any observation; furthermore, the Reuven Ramaty High Energy Solar Spectroscopic Imager solar flare databas $\Phi^{6}$ did not indicate any extreme solar activity within a few days of the observation.

One possibility is that the excess soft flux be due to a rapid, steep-spectrum flare from a transient source in the field of view. Non-detection of the soft emission in the observations obtained on May 18 and 22 suggests that if this is indeed a transient flare, its duration is limited to a maximum of $44 \mathrm{hr}$ considering the stop/start times of the May 18 and 22 observations.

We extracted a spectrum for NGC 4945 combining all the data from May 10-18 and 22-30, and applied the best-fit time-average model (see Table 5) to the joint PCA + HEXTE cluster B spectrum, keeping $\Gamma_{\text {HXPL }}$ and the Fe line centroid energy and width fixed at their time-averaged values. The energy ranges used were $3-26 \mathrm{keV}$ for the PCA and $15-100 \mathrm{keV}$ for HEXTE. A good fit was obtained $\left(\chi^{2} / \mathrm{dof}=59.5 / 74\right)$ without the need to introduce any additional emission components; the total model $2-10 \mathrm{keV}$ flux was $6.8 \times 10^{-12} \mathrm{erg} \mathrm{cm}^{-2} \mathrm{~s}^{-1}$.

We then froze all parameters and applied this model to the PCA + HEXTE cluster B spectrum for the May 20 observation (good exposure times after screening were $1968 \mathrm{~s}$ for the PCA and $541 \mathrm{~s}$ for HEXTE); again, the energy ranges used were 3-26 and 15-100 keV for the PCA and HEXTE, respectively. We added a third power-law component and a Gaussian component to model flaring continuum and Fe K $\alpha$ line emission in excess of that modeled for the nucleus of NGC 4945; more complex models were not necessary to achieve a good fit. The Fe line energy and width $\sigma$ were kept fixed at $6.4 \mathrm{keV}$ and $1 \mathrm{eV}$, respectively. We obtained a good fit $\left(\chi^{2} / \mathrm{dof}=57.6 / 60\right)$ with the following parameters: the photon index of the flaring power law was $2.49_{-0.32}^{+0.37}$ and the normalization at $1 \mathrm{keV}$ was $1.17_{-0.47}^{+0.87} \times 10^{-2} \mathrm{ph} \mathrm{cm}^{-2} \mathrm{~s}^{-1} \mathrm{keV}^{-1}$. The normalization of the Fe line was $7.6 \pm 5.6 \times 10^{-5} \mathrm{ph} \mathrm{cm}^{-2} \mathrm{~s}^{-1}$. The EW of the Fe line with respect to the flaring power-law was $600 \pm 570 \mathrm{eV}$ (taking into account uncertainties on both the Fe line intensity and the normalization of the flaring power law). Removing the Fe line component from the model yielded a fit that was worse by $\Delta \chi^{2}=4.5$ for 1 additional dof. Virtually identical fits were found when the power-law of the flaring emission was absorbed by the total Galactic column $\left(N_{\mathrm{H}, \mathrm{Gal}}=1.4 \times 10^{21} \mathrm{~cm}^{-2}\right)$ or unabsorbed. The best-fit value for the $2-10 \mathrm{keV}$ flux of the flaring power-law was $1.5 \times 10^{-11} \mathrm{erg} \mathrm{cm}^{-2} \mathrm{~s}^{-1}$.

We now discuss possibilities for the origin and nature of the flaring emission. The Galactic latitude and longitude of NGC 4945 are roughly $305^{\circ}$ and $+13^{\circ}$, respectively, i.e., not close enough to either the Galactic center or anti-center to convincingly bolster or diminish the source's likelihood of being Galactic in origin based on sky location alone. There are no sources in the RXTE All-Sky Monitor source catalog in the PCA field of view, though there are numerous unidentified soft X-ray sources within $1^{\circ}$ of the position of NGC 4945 detected with the ROSAT Position Sensitive Proportional Counter (White et al. 2000) and detected in the XMM-Newton Serendipitous Source catalog.

Assuming that the source is located in the host galaxy of NGC 4945, at a luminosity distance of $11.1 \mathrm{Mpc}$, the 2-10 keV luminosity assuming

${ }^{6}$ http://hesperia.gsfc.nasa.gov/hessidata/dbase/hessi_flare_list.txt 
isotropic emission is $2.2 \times 10^{41} \mathrm{erg} \mathrm{s}^{-1}$, which is about $2 / 3$ the $2-10 \mathrm{keV}$ luminosity of the nucleus of NGC 4945 after accounting for absorption (see Table 8). This luminosity is slightly higher than typical X-ray luminosities inferred for ULX sources, $\sim 10^{37-39} \mathrm{erg} \mathrm{s}^{-1}$ (Winter et al. 2006), and so we cannot confirm the emission as originating in a ULX on basis of luminosity alone. In a Suzaku observation of NGC 4945 obtained five months earlier, in 2006 January, Isobe et al. (2008) reported the discovery of the transient ULX source Suzaku J1305-4931 located a few arcmin to the SW of the nucleus of NGC 4945. However, that source's $0.5-10 \mathrm{keV}$ flux was only $\sim 2 \times 10^{-12} \mathrm{erg} \mathrm{cm}^{-2} \mathrm{~s}^{-1}$. As there were no Suzaku observations of NGC 4945 in spring of 2006, it is not clear if the soft excess emission seen in the 2006 May 20 observation can be identified with this ULX.

Assuming instead that the source is located in our Galaxy at a distance which lies in the range $2-15 \mathrm{kpc}$, then the $2-10 \mathrm{keV}$ luminosity is in the range $(0.7-40) \times 10^{34} \mathrm{erg} \mathrm{s}^{-1}$, roughly consistent with many types of Galactic compact accreting sources. Accreting GBH systems, during transition states between the low/hard and high/soft spectral states, can exhibit strong flaring with emission that can be quantified as a power law with $\Gamma \sim 2.0--2.5$ (e.g., Pottschmidt et al. 2003). Strong, rapid (durations of hours) flaring with soft spectra in the $<10 \mathrm{keV}$ range can also be produced by supergiant fast X-ray transients, e.g., Sidoli et al. (2009, and references therein). 